\title{
Large N Duality, Lagrangian Cycles, and Algebraic Knots
}

\section{Citation}

Diaconescu, D.-E., V. Shende, and C. Vafa. 2012. “Large N Duality, Lagrangian Cycles, and Algebraic Knots." Communications in Mathematical Physics 319 (3): 813-63. https:// doi.org/10.1007/s00220-012-1563-3.

\section{Permanent link}

http://nrs.harvard.edu/urn-3:HUL.InstRepos:41385010

\section{Terms of Use}

This article was downloaded from Harvard University's DASH repository, and is made available under the terms and conditions applicable to Open Access Policy Articles, as set forth at http:// nrs.harvard.edu/urn-3:HUL.InstRepos:dash.current.terms-of-use\#OAP

\section{Share Your Story}

The Harvard community has made this article openly available.

Please share how this access benefits you. Submit a story. 


\title{
LARGE $N$ DUALITY, LAGRANGIAN CYCLES, AND ALGEBRAIC KNOTS
}

\author{
D.-E. DIACONESCU ${ }^{1}$, V. SHENDE ${ }^{2}$, C. VAFA $^{3}$
}

\begin{abstract}
We consider knot invariants in the context of large $N$ transitions of topological strings. In particular we consider aspects of Lagrangian cycles associated to knots in the conifold geometry. We show how these can be explicity constructed in the case of algebraic knots. We use this explicit construction to explain a recent conjecture relating study of stable pairs on algebraic curves with HOMFLY polynomials. Furthermore, for torus knots, using the explicit construction of the Lagrangian cycle, we also give a direct A-model computation and recover the HOMFLY polynomial for this case.
\end{abstract}

\section{CONTENTS}

1. Introduction

2. Large $N$ duality and lagrangian cycles for the unknot

2.1. Large $N$ duality for the unknot

2.2. Toric lagrangian cycles in the resolved conifold

3. Algebraic knots, lagrangian cycles and conifold transitions

3.1. Knots and lagrangian cycles in $T^{*} S^{3} \quad 15$

3.2. Lagrangian cycles for algebraic knots 16

3.3. Lagrangian cycles in the resolved conifold 18

4. D-brane bound states and the Hilbert scheme 19

4.1. D2-D0 bound states, vortices, and stable pairs 22

4.2. Intersecting M2-brane bound states 24

4.3. Curves of type $(r, s) \quad 26$

5. A summary for mathematicians 30

6. Large $N$ duality and topological amplitudes for torus knots 33

6.1. Lagrangian cycles for torus knots 34

6.2. Open string A-model on the deformation 36

6.3. Open Gromov-Witten invariants on the resolution 39

6.4. Comparison with HOMFLY polynomial 50

References 52

\section{INTRODUCTION}

The idea that knot invariants can be captured by physical theories dates back to the work of Witten [42] on the relation between Wilson loop observables of ChernSimons quantum field theory for $U(N)$ gauge theories and HOMFLY polynomials. It was later noted by Witten [43] that the Chern-Simons theory, in turn, describes the target space physics of A-model topological strings, in the presence of D-branes. 
In particular, if we have a stack of $N$ D-branes wrapping a three manifold $M^{3} \subset$ $T^{*} M$ viewing $T^{*} M$ as a Calabi-Yau threefold, the large $N$ perturbative Feynman diagrams, i.e. 't Hooft diagrams (known in math literature as 'ribbon graphs') can be viewed as degenerate versions of holomorphic maps from Riemann surfaces with boundaries to $T^{*} M$ where the boundary of the Riemann surface is restriced to lie on $M$. It was later conjectured in 13 that in the special case where $M=S^{3}$ at large $N$ the geometry undergoes a transition, where $S^{3}$ shrinks and an $S^{2}$ is blown up with size equal to $N g_{s}$ where $g_{s}$ is the string (or Chern-Simons) coupling constant. This is the small resolution of the conifold. Furthermore in this new geometry there are no more D-branes. In other words the partition function of the Chern-Simons theory is equivalent to the closed topological A-model involving Riemann surfaces without boundaries, on the resolved conifold. This large $N$ equivalence was checked by computating the partition function on both sides and observing their equality.

One can also extend this equivalence to the computation of the Wilson loop observables for knots, by adding to both sides suitable 'spectator D-branes' 34. Namely for each knot $K \subset S^{3}$, consider the canonical Lagrangian $L_{K} \subset T^{*} S^{3}$ which intersects $S^{3}$ along the knot $K$. Note that $L_{K}$ has the topology $S^{1} \times R^{2}$. The insertion of the spectator brane leads to the insertion of Wilson loop observables on the Chern-Simons side. On the other side the original stack of $N$ D-branes has disappeared but the spectator D-branes $L_{K}$, which have the imprint of the knot, survive. We thus end up with the open topological A-model on the resolved conifold, in the presence of D-branes wrapping $L_{K}$. This equivalence was checked for the unknot in 34. Moreover this equivalence leads to integrality predictions for the coefficients of HOMFLY polynomials (and their colored versions) 34, 22, which has been proven to be true [27, 28, 26]. The integrality structure follows from the fact that on the resolved side the computation of the amplitudes captures the content of BPS particles represented by $\mathrm{M} 2$ branes ending on $L_{K}$, and one is simply counting them. For example, for the unknot the partition function is captured by the fact that there are two M2 branes ending on $L_{K}$.

This leaves open the problem of directly computing the topological A-model for the resolved conifold in the presence of branes wrapping $L_{K}$. The difficulty in performing this task is two-fold: first we have to identify the Lagrangian subspace $L_{K}$, and second set up a computation for the A-model amplitudes. The difficulty with the first task is that before transition $L_{K}$ intersects $S^{3}$, and thus as $S^{3}$ shrinks $L_{K}$ becomes singular and its continuation on the resolved side is delicate (though there has been progress along these lines in 41, 21. However, it was further noted in 29] that to make this more well defined, and also in order for the framing dependence to come out accurately we need to lift the original Lagrangian $L_{K}$, so that it no longer touches the $S^{3}$, but is seperated from it by a cylinder which ends on the one hand on the knot in $S^{3}$ and on the other to the non-trivial circle in $L_{K}$. In this way the $L_{K}$ is non-singular as $S^{3}$ shrinks and the process of identifying it on the resolved side is more straight-forward. We will clarify this construction later in this paper.

The second task is to compute the A-model amplitudes. When there are enough symmetries this in principle can be done in two ways: Either by direct computation using localization techniques, or by enumerating BPS particles ending on $L_{K}$.

The enumeration of BPS particles correponding to M2 branes ending on $L_{K}$ is particularly simple for a special knots, including the unknot. For example for the 
the unknot there are two BPS particles. One corresponds to a disc which lives on the $\mathbf{C}^{2}$ fiber of one point in $\mathbf{P}^{1}$. The disc intersects $L_{K}$ on a circle where it ends. The other particle is made of the bound state of this disc with an M2 brane wrapping $\mathbf{P}^{1}$. This follows from the fact that the binding process is local, and we already have the disc ending on $L_{K}$ and the closed M2 brane on $\mathbf{P}^{1}$ each as BPS states, and they intersect transversally (relative codimension 4). Thus they form a unique bound state. From this we can recover the HOMFLY polynomial for the unknot. In some sense the unknot is 'planar' in that the BPS structures are captured by objects living on the fiber or on the base independently, and simply glued together.

The question remains as for which knots are 'planar' in this sense? The natural answer ends up being the class of knots known as algebraic knots, which can be defined by holomorphic function of two variables. One considers in complex dimension 2 a holomorphic function $f(x, y)$ with a singularity structure at the origin. The intersection of

$$
f(x, y)=0
$$

with a large 3 -sphere

$$
|x|^{2}+|y|^{2}=r
$$

for large $r$ gives a knot $K_{f}$ on $S^{3}$. It turns out that for these knots the corresponding Lagrangian $L_{K}$ can be constructed explicitly. Moreover, just as in the case of the unknot they are 'planar'. In particular, the primitive holomorphic curve ending on it lives on a fiber over a single point of $\mathbb{P}^{1}$. Moreover, identifying the fiber with complex coordinates $(x, y)$ the basic holomorphic curve for the M2 brane is exactly $f(x, y)=0$ and it intersects $L_{K}$ on the large three sphere along an $S^{1}$. The new novely, as compared to the case of unknot, is that there could be more than one M2 brane bound state on $f(x, y)=0$ curve. Enumeration of such bound states turns out to map to a math problem recently studied in [32. However in the more general case, we have more possibilities for forming bound states, not just the single disc as in the case of the unknot. Furthermore, just as in the case of the unknot, for each such disc we can form bound states of this open $M 2$ brane with a closed M2 brane wrapping $\mathbf{P}^{1}$. The number of bound states depends on the intersection number of the $\mathbf{P}^{1}$ with the corresponding transverse bound state. For each intersection point, we get a bound state. Considering all such BPS states wrapping the fiber and base we get the enumeration of BPS states in this geometry which leads to the evaluation of the HOMFLY polynomial for such knots. This turns out to explain the conjecture of 32 relating the HOMFLY polynomial for algebraic knots with computations done for stable pairs associated to the corresponding curve.

We explain in detail how these bound states can be evaluated for the case of the torus knots where

$$
f(x, y)=x^{r}-y^{s} .
$$

Furthermore, for these cases, using the explicit construction of the Lagrangian cycles $L_{K}$ we are able to also directly compute the A-model amplitudes as well and rederive the HOMFLY polynomials for torus knots.

The organization of this paper is as follows. Section two is a review of large $N$ duality for the unknot, including the construction of toric lagrangian cycles on the resolved conifold. The main goal of this discussion is to motivate the general idea of lifting conormal bundle lagrangian cycles in the deformed conifold. Section three presents an explicit construction of such a lift for algebraic knots, as well as 
the corresponding lagrangian cycles in the resolved conifold. Section four provides a physical explanation for the conjecture of Oblomkov and Shende 32] relating HOMFLY polynomials of of algebraic knots to certain generating functions associated to Hilbert schemes of plane curve singularities. In particular, the generating functions employed in 32 are identified with counting functions for open M2-brane microstates with boundary on an M5-brane wrapping a lagrangian cycle. Section five is a reprise of section four in more mathematical dialect. Finally, section six consists of detailed computations of open topological A-model amplitudes for lagrangian cycles corresponding to $(s, r)$-torus knots. The main result is a geometric derivation of the Chern-Simons $S$-matrix formula found in [39, 5] by manipulations of open Gromov-Witten invariants.

Acknowledgments. We thank Lev Borisov, Wu-yen Chuang, Zheng Hua, Amer Iqbal, Melissa Liu, Sheldon Katz, Alexei Oblomkov, Andrei Okounkov, Rahul Pandharipande, Tony Pantev, Paul Seidel, Clifford Taubes, Richard Thomas, Yan Soibelman and Chris Woodward for very helpful discussions. D.-E. D. and V.S. would especially like to thank Alexei Oblomkov for collaboration on related projects and many insightful discussions. The work of D.-E.D. was partially supported by NSF grant PHY-0854757-2009. V.S. was supported by an EPSRC programme grant on a visit to Imperial college, and is currently supported by the Simons foundation. The work of C.V. is supported in part by NSF grant PHY-0244821. We would also like to thank the 2010 and 2011 Simons workshop in Mathematics and Physics and the Simons Center for Geometry and Physics for hospitality during the inception of this work. C.V. would also like to acknowledge the MIT physics department for hospitality.

\section{LARGE $N$ DUALity AND LAGRANGIAN CYClES FOR THE UNKNOT}

The conifold transition is a topology changing process relating the smooth hypersurface $X_{\mu}$

$$
x z-y w=\mu
$$

in $\mathbb{C}^{4}$ with $\mu \in \mathbb{C} \backslash\{0\}$ to the small resolution $Y$ of the singular threefold $X_{0}$ obtained at $\mu=0$. In fact there exist two such isomorphic resolutions related by a toric flop. For concreteness, let $Y$ be the resolution obtained by blowing-up the subspace $y=z=0$ in $\mathbb{C}^{4}$. Then $Y$ is determined by the equations

$$
x \lambda=w \rho, \quad z \rho=y \lambda
$$

in $\mathbb{C}^{4} \times \mathbb{P}^{1}$ and there is a natural map $\sigma: Y \rightarrow X_{0}$ which contracts the rational curve $y=z=0$ on $Y$. It can be easily seen that $Y$ is isomorphic to the total space of the rank two bundle $\mathcal{O}_{\mathbb{P}^{1}}(-1) \oplus \mathcal{O}_{\mathbb{P}^{1}}(-1)$ and the curve $y=z=0$ is identified with its zero section, which is the only compact holomorphic curve on $Y$

The deformed conifold $X_{\mu}, \mu \neq 0$, equipped with the symplectic form

$$
\omega_{X_{\mu}}=\left.\omega_{\mathbb{C}^{4}}\right|_{X_{\mu}}, \quad \omega_{\mathbb{C}^{4}}=\frac{i}{2}(d x \wedge d \bar{x}+d y \wedge d \bar{y}+d z \wedge d \bar{z}+d w \wedge d \bar{w})
$$

is symplectomorphic to the total space $X$ of the cotangent bundle $T^{*} S^{3}$. For $\mu \in \mathbb{R}_{>0}$, this can be seen explicitly [37, 38] observing that equation (2.1) becomes

$$
\sum_{i=1}^{4} z_{i}^{2}=\mu
$$


in the coordinates

$$
x=z_{1}+i z_{2}, \quad z=z_{1}-i z_{2}, \quad y=-z_{3}-i z_{4}, \quad w=z_{3}-i z_{4} .
$$

Writing $z_{j}=x_{j}+i y_{j} j=1, \ldots, 4$, with $\left(x_{j}, y_{j}\right)$ real coordinates on $\mathbb{C}^{4}$, equation (2.1) is further equivalent to

$$
\vec{x} \cdot \vec{y}=0, \quad|\vec{x}|^{2}-|\vec{y}|^{2}=\mu .
$$

Here - denotes the Euclidean inner product on $\mathbb{R}^{4}$ and || the Euclidean norm.

On the other hand, the total space $X$ of the cotangent bundle $T^{*} S^{3}$ is identified with the subspace $\{(\vec{u}, \vec{v})\} \subset \mathbb{R}^{4} \times \mathbb{R}^{4}$ satisfying

$$
|\vec{u}|=1, \quad \vec{u} \cdot \vec{v}=0
$$

The canonical symplectic form on $X=T^{*} S^{3}$ is then obtained by restriction from the ambient space,

$$
\omega_{X}=\left.\left(\sum_{j=1}^{4} d v_{j} \wedge d u_{j}\right)\right|_{X}
$$

According to equation (2.3),$\vec{x} \neq 0$ on $X_{\mu}$ since $\mu \in \mathbb{R}_{>0}$. Therefore there is a well defined map

$$
\phi_{\mu}: X_{\mu} \rightarrow X \quad \phi_{\mu}(\vec{x}, \vec{y})=\left(\frac{x_{j}}{|\vec{x}|},-|\vec{x}| y_{j}\right)
$$

It is straightforward to check that this map is a diffeomorphism, its inverse being given by

$$
\phi_{\mu}^{-1}(\vec{u}, \vec{v})=\left(f_{\mu}(\vec{v}) \vec{u},-f_{\mu}(\vec{v})^{-1} \vec{v}\right), \quad f_{\mu}(\vec{v})=\sqrt{\frac{\mu+\sqrt{\mu^{2}+4|\vec{v}|^{2}}}{2}} .
$$

It is also straightforward to check that

$$
\phi_{\mu}^{*}\left(\omega_{X}\right)=\left.\frac{i}{2} \omega_{\mathbb{C}^{4}}\right|_{X_{\mu}}
$$

Therefore $\phi_{\mu}$ is indeed a symplectomorphism.

A similar construction yields a symplectomorphism $\phi_{0}: X_{0} \backslash\{0\} \rightarrow X \backslash\{\vec{v}=0\}$ between the complement of the singular point in $X_{0}$ and the complement of the zero section in $X=T^{*} S^{3}$. Observing that $\vec{x} \neq 0$ on $X_{0} \backslash\{0\}, \phi_{0}$ is given exactly by the same formula as $\phi_{\mu}, \mu>0$. The same computation shows that $\phi_{0}$ is a symplectomorphism if $X_{0} \backslash\{0\}$ is equipped with the symplectic structure obtained by restriction from $\mathbb{C}^{4}$.

Note also that there is an antiholomorphic involution

$$
(x, y, z, w) \mapsto(\bar{z},-\bar{w}, \bar{x},-\bar{y})
$$

on $\mathbb{C}^{4}$ which preserves $X_{\mu}$ with $\mu \in \mathbb{R}_{\geq 0}$. Therefore there are induced antiholomorphic involutions $\tau_{\mu}: X_{\mu} \rightarrow X_{\mu}, \mu \in \mathbb{R}_{\geq 0}$. For $\mu>0$, the fixed locus $S_{\mu}$ of $\tau_{\mu}$ is isomorphic to the three-sphere $|x|^{2}+|z|^{2}=\mu$ in $\mathbb{C}^{2}$. By construction, $S_{\mu}$ is a special lagrangian cycle on $X_{\mu}$ and the image $\phi_{\mu}\left(S_{\mu}\right)$ is the zero section $S=\{\vec{v}=0\}$ of the cotangent bundle $T^{*} S^{3}$. 
2.1. Large $N$ duality for the unknot. The primary example of large $N$ duality for topological strings [13] is an equivalence between the large $N$ limit of the topological A-model on $X_{\mu}$ with $N$ lagrangian branes on $S_{\mu}$ and the topological A-model on $Y$. The partition function of the latter is given by

$$
Z_{Y}(q, Q)=\prod_{n \geq 1}\left(1-Q(-q)^{n}\right)^{n}
$$

where $q$ and $Q$ are related to the string coupling constant $g_{s}$ and the symplectic area $t_{0}$ of $C_{0}$ by $q=e^{i g_{s}}, Q=e^{-t_{0}}$.

According to 43 the topological A-model on $X_{\mu}$ with $N$ lagrangian branes on the sphere $S_{\mu}$ is equivalent to $U(N)$ Chern-Simons theory on $S_{\mu}$. The level $k$ of the Chern-Simons theory is related to the string coupling constant. The partition function of the Chern-Simons theory on $S_{\mu}$ is naturally expanded in terms of the large $N$ variables

$$
g_{s}=\frac{2 \pi}{k+N}, \quad \lambda=\frac{2 \pi N}{k+N} .
$$

Then large $N$ duality [13] suggests that the theory on $S_{\mu}$ where there is brane, is equivalent to the one after geometric transition where the branes have disappeared and replaced by a blown up 2-sphere. This duality thus identifies the analytic part of the Chern-Simons large $N$ expansion with the closed topological string amplitude $Z_{Y}(q, Q)$ on the resolved side]

$$
Z_{C S}\left(g_{s}, \lambda\right)=\left.Z_{Y}(q, Q)\right|_{q=e^{i g_{s}}, Q=e^{i \lambda}}
$$

Large $N$ duality has been extended to Chern-Simons theory with Wilson loops in [34. The main idea is that given a smooth knot $K \subset S^{3}$ the total space $L$ of the conormal bundle $N_{K}^{*}$ to $K$ in $S^{3}$ is a lagrangian cycle in $X=T^{*} S^{3}$. Since $\phi_{\mu}$ : $X_{\mu} \rightarrow X$ is a symplectomorphism, the inverse image $L_{\mu}=\phi_{\mu}^{-1}(L)$ is a lagrangian cycle on $X_{\mu}$. According to 34, a configuration of $N$ branes on $S_{\mu}$ and $M$ branes on $L_{\mu}$ has a complex bosonic open string mode localized on their intersection which transforms in the bifundamental representation of $U(N) \times U(M)$. Integrating out this mode yields a series of Wilson line corrections to Chern-Simons theory on $S$ of the form

$$
\sum_{n \geq 1} \frac{1}{n} \operatorname{Tr}\left(U^{n}\right) \operatorname{Tr}\left(V^{-n}\right)
$$

Here $U$ is the holonomy of the Chern-Simons gauge field $A$ on $K$ and $V$ is the holonomy on $K$ of an arbitrary background flat gauge field on $L_{\mu}$. This integrating out can also be explained in terms of the annulus contributions to the amplitudes where one boundary of the annulus ends on $S_{\mu}$ and the other ends on $L_{\mu}$. These are 'holomorphic' annuli which have zero width, corresponding to the fact that in the dual channel there are massless bi-fundamental particles of $U(N) \times U(M)$ going in the loop.

Therefore in the presence of the $M$ noncompact branes on $L_{\mu}$, the (analytical part of the) topological open string partition function becomes

$$
Z_{C S}\left(g_{s}, \lambda\right)\left\langle\exp \left(\sum_{n \geq 1} \frac{1}{n} \operatorname{Tr}\left(U^{n}\right) \operatorname{Tr}\left(V^{-n}\right)\right)\right\rangle
$$

\footnotetext{
${ }^{1}$ The non-analytic part of the Chern-Simons function can be identified by the same change of variables with the polynomial part of the $N=2$ prepotential of a IIA compactification on $Y$.
} 
where \langle\rangle denote the expectation values of Wislon line operators in $U(N)$ ChernSimons theory on $S^{3}$. The main question is then to construct a dual topological string model on the resolution $Y$, extending the results of [13].

This problem was solved in 34 for the case when $K$ is the unknot. For concreteness let $K \subset S^{3}$ be determined by the equations

$$
y=w=0, \quad|x|=|z|=\sqrt{\mu}
$$

on $X_{\mu}$. Omitting the details, a straightforward computation shows that the inverse image $\phi_{\mu}^{-1}\left(N_{K}^{*}\right)$ is the lagrangian cycle $L_{\mu}$ in $X_{\mu}$ determined by the equations

$$
y=\bar{w}, \quad|x|=|z| .
$$

Assuming $K$ to be trivially framed, the large $N$ expansion of the partition function (2.10) is in this case

$$
Z_{C S}\left(g_{s}, \lambda\right) \exp \left[-i \sum_{n \geq 1} \frac{e^{i n \lambda / 2}-e^{-i n \lambda / 2}}{2 n \sin \left(n g_{s} / 2\right)} \operatorname{Tr}\left(V^{-n}\right)\right] .
$$

In order to find a large $N$ duality interpretation, note that the above partition function is related by analytic continuation to

$$
Z_{C S}\left(g_{s}, \lambda\right) \exp \left[-i \sum_{n \geq 1} \frac{\operatorname{Tr}\left(V^{n}\right)+\operatorname{Tr}\left(V^{-n}\right)}{2 n \sin \left(n g_{s} / 2\right)} e^{i n \lambda / 2}\right] .
$$

This expression is then identified with a series of open Gromov-Witten invariants of a lagrangian cycle $M$ in $Y$ determined by the equations

$$
|\lambda|=|\rho|, \quad x \lambda=\bar{y} \rho .
$$

By construction, $M$ intersects the zero section $C_{0}$ along the circle $|\lambda|=|\rho|$, dividing it into two discs $\mathrm{D}_{ \pm}$with common boundary. The terms weighted by $\operatorname{Tr}\left(V^{n}\right)$, $\operatorname{Tr}\left(V^{-n}\right)$ in the in the exponent of (2.14) represent open Gromov-Witten invariants with positive, respectively negative winding numbers along the circle $|\lambda|=|\rho|$. This was confirmed by virtual localization computations in [18, 23]. In particular, the terms with positive winding numbers are obtained by summing over multicovers of $\mathrm{D}_{+}$while those with negative winding numbers are obtained from multicovers of $\mathrm{D}_{-}$.

The main difficulty in extending the above results to more general knots in $S^{3}$ resides in the identification of the lagrangian cycle $M$ in $Y$ associated to a given knot $K$. Ideally there should be a natural geometric relation between the cycle $M \subset Y$ and the specialization $L_{0} \subset X_{0}$ of $L_{\mu} \subset X_{\mu}$ as $\mu \rightarrow 0$, exploiting the fact that the conifold transition is a basic example of symplectic surgery [38, 37]. In symplectic geometry the blow-up of $X_{0}$ as a symplectic manifold depends on a positive real parameter $\epsilon \in \mathbb{R}_{>0}$ which measures the symplectic area of the exceptional curve $C_{0} \subset Y$. More precisely let $\omega_{0}$ denote the symplectic form $\left.\omega_{\mathbb{C}^{4}}\right|_{X_{0} \backslash\{0\}}$ on the complement of the conifold singularity in $X_{0}$. Then the blow-up of $X_{0}$ is a family of symplectic Kähler manifolds $Y_{\epsilon}=\left(Y, \omega_{Y, \epsilon}\right)$ such that the resulting family of symplectic Kähler forms $\left.\omega_{Y, \epsilon}\right|_{Y \backslash\left\{C_{0}\right\}}$ on the complement of $C_{0}$ degenerates to $\sigma^{*} \omega_{0}$ at $\epsilon=0$. This yields a more symmetric picture of the conifold transition transition, involving two families of symplectic manifolds $X_{\mu}, Y_{\epsilon}$ satisfying a natural 
compatibility condition at $\mu=0, \epsilon=0$ respectively. This process is schematically summarized by the following diagram.

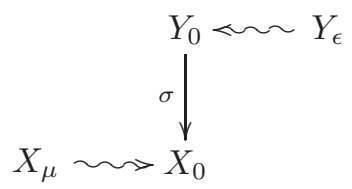

where $\sigma: Y_{0} \rightarrow X_{0}$ is the blow-up map. Note that all $Y_{\epsilon}$ with $\epsilon \geq 0$ are identical as complex manifolds, but not as symplectic manifolds. The symplectic structure is degenerate at $\epsilon=0$ since $C_{0}$ has zero symplectic area with respect to $\omega_{0}$.

In this framework, a natural formulation of large $N$ duality for knots requires two families of lagrangian cycles $X_{\mu} \subset X_{\mu}, M_{\epsilon} \subset Y_{\epsilon}$ such that the degenerations $L_{0}, M_{0}$ are related by $M_{0}=\sigma^{*} L_{0}$, at least on the complement of the exceptional curve $C_{0}$. Schematically, such a process would be captured by an enhanced diagram

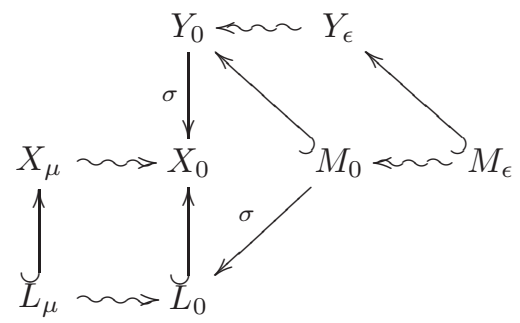

In the case of the unknot reviewed above, the specialization of the cycle $L_{\mu}$ in equation (2.12) at $\mu=0$ is the singular lagrangian cone $L_{0} \subset X_{0}$ determined by

$$
y=\bar{w}, \quad|x|=|y| .
$$

At the same time, the cycle $M$ constructed in equation (2.15) is lagrangian with respect to any symplectic Kähler form $\omega_{Y, \epsilon}$ because it is the fixed point set of an antiholomorphic involution. The image of $M$ via the blow-up map $\sigma$ is precisely the singular lagrangian cycle $L_{0}$ determined by the same equations (2.18). Therefore the compatibility condition at $\mu=0, \epsilon=0$ is satisfied. For illustration, the resulting geometric picture is represented in figure (11).

Since the knot $K$ is contracted in this process it is not clear how such a construction can be extended to more general knots especially such that the resulting open string Gromov-Witten theory on $Y$ is tractable. A related problem is that the analytic continuation required by a proper enumerative interpretation of the partition function does not have a direct geometric interpretation.

Both these problems lead to the idea [29, 2] that a better formulation of large $N$ duality would be obtained using lagrangian cycles supported in the complement of the zero section $C_{0} \subset Y$, respectively $S^{3} \subset X_{\mu}$. Said differently, this means that the lagrangian cycle $L=N_{K}^{*}$ must be lifted to a lagrangian cycle disjoint from the zero section prior to the transition. Accordingly, the corresponding lagrangian cycle in $Y$ will be lifted to a cycle disjoint from the zero section $C_{0}$. Moreover, once properly lifted, these cycles should form families naturally related by symplectic surgery as explained above. That there is such a lift can be argued as follows: Assume that with the proper choice of metric, the lagrangian cycle $L$ is actually special lagrangian [40. In this case it is known that the dimension of moduli of $L$ is equal to the dimension of $H_{1}$. This is rigorously the case for compact lagrangians, 

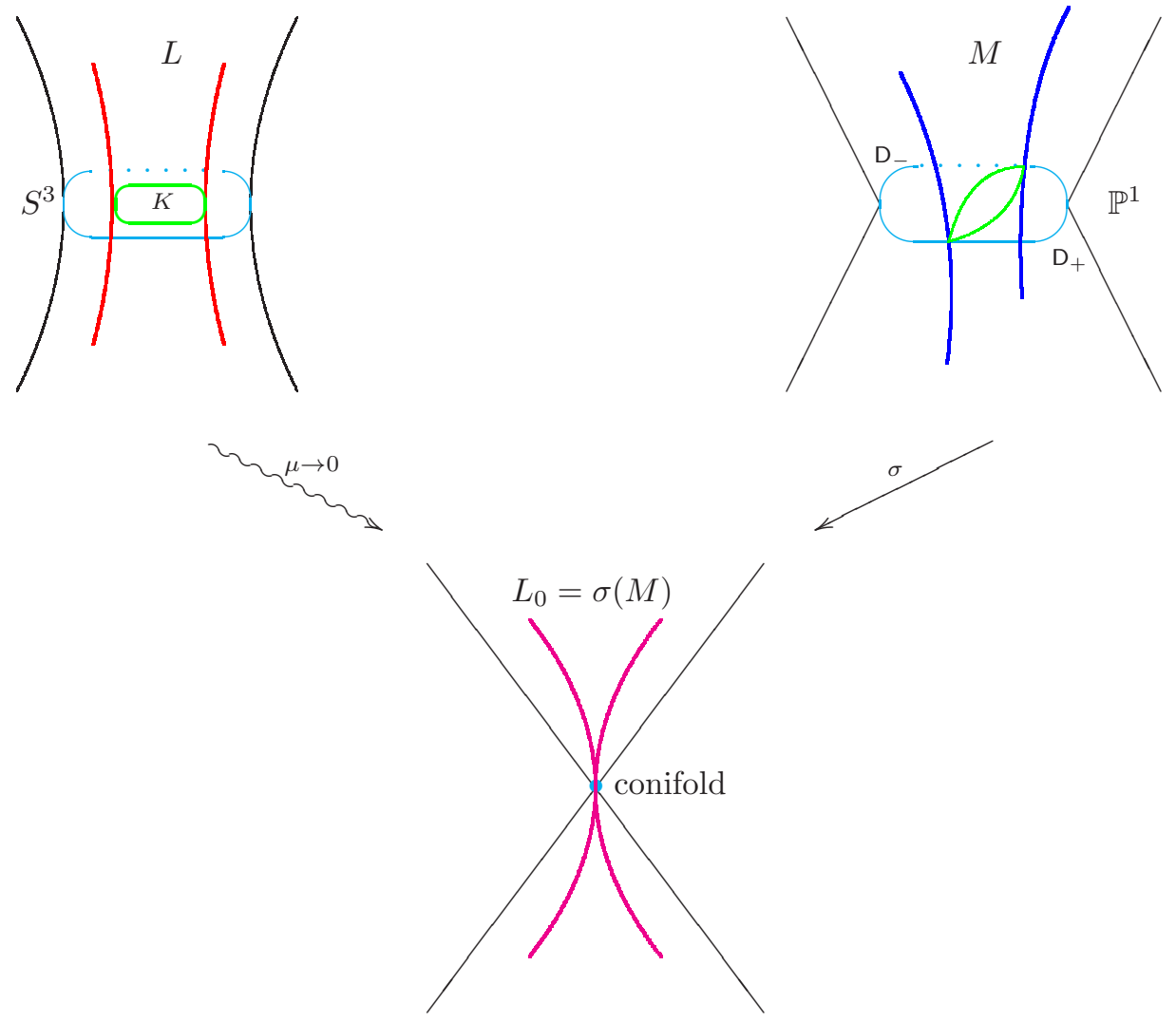

Figure 1. Conifold transition for unlifted lagrangian cycles.

and we assume it to hold for non-compact ones as well where we have imposed sutiable finiteness conditions on the norm of deformations of the lagrangian. Since the topology of $L$ is $R^{2} \times S^{1}$, there is exactly 1 deformation. This corresponds to moving the special lagrangian in the 1 -form dual the $S^{1}$, by identifying the infinitesimal normal deformation to the lagrangian with its cotangent space. It is this deformation that lifts the $L$ off of $S_{\mu}$. Moreover it suggests that there is a unique such canonical lift for special lagrangian cycles. Even though we will mainly deal with just lagrangian ones, this suggests that the choice of the special lagrangian ones make the constructions more 'canonical'.

Accepting the idea of lifting lagrangian cycles, a legitimate question is how can one then obtain the Wilson loop corrections (2.9), given that $L_{\mu}$ and $S_{\mu}$ do not intersect. This is also natural. Lifting off of the lagrangian brane off of $S_{\mu}$ is simply giving the bi-fundamental particles a mass given by the amount of lifting. In other words, the annuli which whose dual channel corresponded to bi-fundamental strings, now have a finite width depending on the amount of lift. These corrections can now be interpreted as 'honest' intantons, i.e. holomorphic cylinders which on the one hand end on $S_{\mu}$ and on the other hand on $L_{\mu}$. Such corrections were predicted 

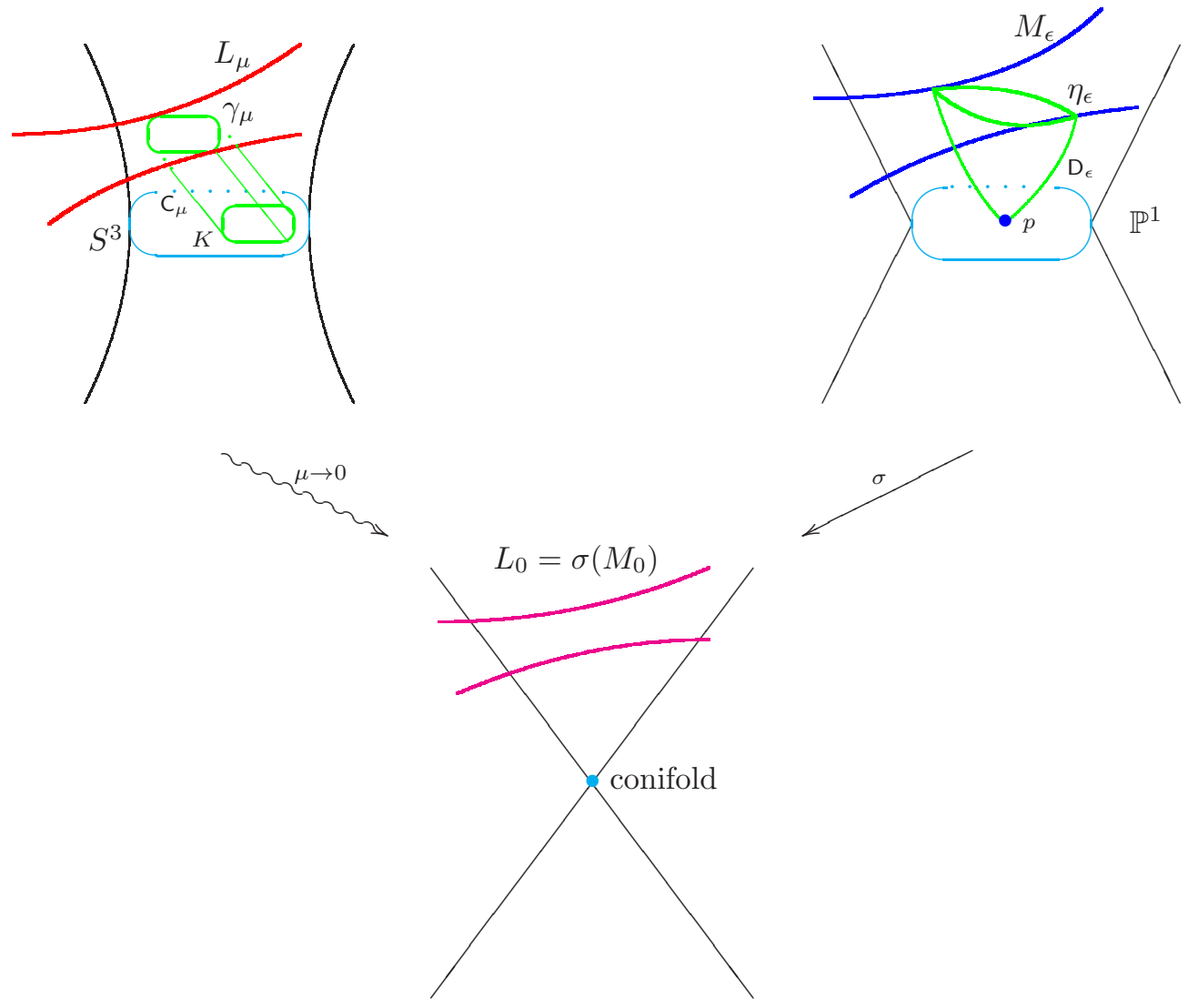

Figure 2. Conifold transition for lifted lagrangian cycles.

in 43 assuming that there are finitely many rigid holomorphic Riemann surfaces $\mathrm{C}_{\mu}^{(\alpha)}$ in $X_{\mu}$ with boundary components on $S_{\mu}, L_{\mu}$. Each such surface gives rise to a series of Wilson loop corrections by summing over multicovers. In particular, a rigid holomorphic cylinder $\mathrm{C}_{\mu}$ in $X_{\mu}$ with boundary components in $S_{\mu}, L_{\mu}$ yields a series of instanton corrections

$$
\sum_{n \geq 1} \frac{e^{-t_{C}}}{n} \operatorname{Tr}\left(U^{n}\right) \operatorname{Tr}\left(V^{n}\right)
$$

where $t_{C}$ is the symplectic area of the cylinder $C_{\mu}$, and can be interpreted as the mass of the bi-fundamental state (where we have changed the variables by $V \mapsto V^{-1}$ ). Note that the factor $e^{-t_{c}}$ can be absorbed by a redefinition of the holonomy variable $V$, hence it will be omitted from now on. Figure (2) is a schematic representation of the surgery process in terms of lifted lagrangian cycles.

To summarize, for a given knot $K \in S^{3}$, large $N$ duality requires a family of lagrangian cycles $L_{\mu} \subset X_{\mu}$, disjoint from $S_{\mu}$, such that there is a unique rigid holomorphic holomorphic cylinder $\mathrm{C}_{\mu}$ in $X_{\mu}$ with boundary components in $S_{\mu}, L_{\mu}$. Moreover, the boundary component in $S_{\mu}$ must be isotopic to the given knot $K$. 
Note that the rigidity assumption on $\mathrm{C}_{\mu}$ is not needed if there exists a torus action on $X_{\mu}$ preserving $L_{\mu}$. In this case it suffices to require $\mathrm{C}_{\mu}$ to be the unique torus invariant holomorphic cylinder satisfying these boundary conditions. Then the series (2.19) follows by a virtual localization computation analogous to [18, as shown for example in [10. A concrete construction of such families of lagrangian cycles for algebraic knots is presented in section (3). By analogy with the unknot, the cycles $L_{\mu}$ will be obtained by taking inverse images $\phi_{\mu}^{-1}(L)$ of a fixed lift $L \subset X$ of $N_{K}^{*}$ in $T^{*} S^{3}$. Uniqueness and rigidity of the associated holomorphic cylinders will be proven only for torus knots in section (6) and conjectured to hold for all algebraic knots.

The family of lagrangian cycles $M_{\epsilon} \subset Y$ related to $L_{\mu}$ by geometric transition is expected to have a similar property. Namely there should exist a unique holomorphic disc $\mathrm{D}_{\epsilon}$ in $Y$ with boundary $\eta_{\epsilon} \subset M_{\epsilon}$. Note that $\mathrm{D}_{\epsilon}$ may have isolated singularities away from the boundary. Again, if there is a torus action on $Y$ preserving $M_{\epsilon}$, it suffices for $\mathrm{D}_{\epsilon}$ to be the unique torus invariant disc with boundary on $M_{\epsilon}$. Then large $N$ duality predicts an identification between the Chern-Simons partition function on $S^{3}$, including the instanton corrections (2.19), and the partition function of Gromov-Witten theory on $Y$ with lagrangian boundary conditions on $M_{\epsilon}$.

As a first example, the above program will be carried out in detail in the next subsection for an unknot of the form (2.11). In this case the cycles $M_{\epsilon}, L_{\mu}$ will be explicitly constructed employing toric methods 4 It will be shown that both cycles are preserved by a circle action determined by an action on $\mathbb{C}^{4}$ of the form

$$
(x, y, z, w) \mapsto\left(e^{-i n_{1} \varphi} x, e^{-i n_{2} \varphi} y, e^{i n_{1} \varphi} z, e^{i n_{2} \varphi} w\right) .
$$

Note that the action on $Y$ is uniquely determined by the condition that the blow-up equations (2.2) be left invariant. In particular it yields the circle action

$$
[\lambda, \rho] \mapsto\left[\lambda, e^{i\left(n_{1}+n_{2}\right) \varphi} \rho\right]
$$

on $\mathbb{P}^{1}$. Assuming the unknot trivially framed, the Chern-Simons expectation value of the instanton corrections (2.19) is

$$
\exp \left[i \sum_{n \geq 1} \frac{\left(1-e^{i n \lambda}\right)}{2 n \sin \left(n g_{s} / 2\right)} \operatorname{Tr}\left(V^{-n}\right)\right]
$$

The open Gromov-Witten invariants with boundary condition on $M_{\epsilon}$ can be computed in close analogy with [18. As explained in [18, the result depends on the choice of a torus action, reflecting the fact that the moduli space of stable maps with lagrangian boundary conditions is non-compact. This dependence is related by large $N$ duality to the framing dependence of knot invariants in Chern-Simons theory [29]. Choosing the torus action $\left(n_{1}, n_{2}\right)=(1,0)$, which corresponds to the trivial framing, the result takes the simple form

$$
\exp \left[i \sum_{n \geq 1} \frac{\left(1-Q^{n}\right)}{2 n \sin \left(n g_{s} / 2\right)} \operatorname{Tr}\left(V^{-n}\right)\right]
$$

This is in agreement with equation (2.21) via the change of variable $Q=e^{i \lambda}$. Note that the term involving a single $\operatorname{Tr}\left(V^{-1}\right)$ in the exponent has the form (up to an 
overall factor of $q^{1 / 2}$ )

$$
(1-Q) /(1-q)=\frac{1}{(1-q)}-\frac{Q}{(1-q)}
$$

where $q=\exp \left(i g_{s}\right)$. Each of these two terms was interpreted in [34] as the contribution of an M2 brane ending on the Lagrangian brane corresponding to the unknot. The two term differ by a factor of $Q$ indicating that one of the two M2 branes is in addition wrapped around the $\mathbf{P}^{1}$. The minus sign in front of the second term can be interpreted as the fermion number associated with the M2 wrapped around $\mathbf{P}^{1}$. Moreover the term

$$
\frac{1}{(1-q)}=1+q+q^{2}+\ldots
$$

signifies the fact that an M2 brane particle has one mode for each positive integer $n>0$. Each such $n$ corresponds to the spin of the M2 brane on a plane, in the presence of a magnetic flux. Moreover in the type IIA perspective since the rotation around the 11-th circle is identified with the rotation on the 2-plane, $n$ can also be identified with the D0 brane charge [1, 3, 6]. The fact that there are two BPS states for the unknot will be explained in the next subsection.

2.2. Toric lagrangian cycles in the resolved conifold. The construction of the lifted lagrangian cycles $M_{\epsilon}, L_{\mu}$ will be carried out in detail below for the unknot using toric geometry as in [4. The gauged linear sigma model which flows to $Y$ is a two dimensional $U(1)$ gauge theory containing four chiral superfields $Z_{1}, \ldots, Z_{4}$ with charges

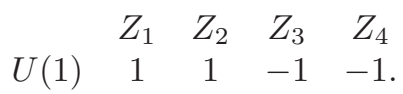

and trivial superpotential. The $\mathrm{D}$-term equation is

$$
\left|Z_{1}\right|^{2}+\left|Z_{2}\right|^{2}-\left|Z_{3}\right|^{2}-\left|Z_{4}\right|^{2}=\epsilon,
$$

where $\epsilon \in \mathbb{R}_{>0}$ is an FI parameter. The symplectic quotient construction yields a family of symplectic Kähler manifolds $Y_{\epsilon}=\left(Y, \omega_{Y, \epsilon}\right)$. The exceptional curve $C_{0}$ is given by $Z_{3}=Z_{4}=0$, and has symplectic area proportional to $\epsilon$. The contraction map $\sigma: Y \rightarrow X_{0}$ is determined by the $U(1)$-invariant monomials

$$
x=Z_{3} Z_{1}, \quad y=Z_{4} Z_{1}, \quad z=Z_{4} Z_{2}, \quad w=Z_{3} Z_{2}
$$

which satisfy the relation $x z=y w$.

Lagrangian cycles in $Y$ are constructed by a linear gauged linear sigma model with boundary, which is expected to flow to a boundary conformal field theory in the infrared. In particular consider the cycles $M_{\epsilon}$ be defined by the boundary D-term equations

$$
\left|Z_{2}\right|^{2}-\left|Z_{4}\right|=0, \quad\left|Z_{3}\right|^{2}-\left|Z_{4}\right|^{2}=c,
$$

where $c \in \mathbb{R}_{>0}$ is a boundary FI parameter, and the phase condition

$$
Z_{1} \cdots Z_{4}=\left|Z_{1} \cdots Z_{4}\right| \text {. }
$$

On the open subset $Z_{i} \neq 0$, where all angular coordinates $\theta_{i}, i=1, \ldots, 4$, are well defined this condition is equivalent to $\theta_{1}+\cdots+\theta_{4}=0$. A detailed construction of the boundary gauged linear sigma models has been carried out in [16, 30, 14, 15. The boundary FI parameter $c>0$ will be kept fixed throughout this discussion. 
In order to understand the geometry of $M_{\epsilon}$, note that equations (2.23), (2.24) imply

$$
\left|Z_{1}\right|^{2}-\left|Z_{3}\right|^{2}=\epsilon
$$

Since $\xi, c>0$, it follows that $Z_{1}, Z_{3}$ cannot vanish on $M_{\epsilon}$. Then the phase $\theta_{1}$ can be set to 0 by $U(1)$ gauge transformations, and the phase relation (2.25) reduces to

$$
Z_{2} \cdots Z_{4}=\left|Z_{2} \cdots Z_{4}\right|
$$

As emphasized in the previous subsection, it is important to note that $M_{\epsilon}$ is preserved by any circle action $S^{1} \times Y \rightarrow Y$ of the form

$$
\left(Z_{1}, \ldots, Z_{4}\right) \mapsto\left(Z_{1}, e^{i\left(n_{1}+n_{2}\right) \varphi} Z_{2}, e^{-i n_{1} \varphi} Z_{3}, e^{-i n_{2} \varphi} Z_{4}\right)
$$

with $n_{1}, n_{2} \in \mathbb{Z}$. It is straightforward to check that this is in agreement with the action (2.20) on the invariant monomials. It is also important to note that $M_{\epsilon}$ intersects the plane $Z_{2}=0$ along a circle $S_{c}^{1}$ given by

$$
\left|Z_{2}\right|=\left|Z_{4}\right|=0, \quad\left|Z_{3}\right|^{2}=c, \quad\left|Z_{1}\right|^{2}=\epsilon+c .
$$

Since $Z_{2}, Z_{4}$ are set to 0 , the intersection is indeed a circle parameterized by the angular variable $\theta_{3}$. Moreover, there is a holomorphic disc $D_{\epsilon}$ with boundary on $M_{\epsilon}$ defined by

$$
\left|Z_{2}\right|=\left|Z_{4}\right|=0, \quad\left|Z_{3}\right|^{2} \leq c, \quad\left|Z_{1}\right|^{2}=\epsilon+c .
$$

Reasoning by analogy with [9, 10, it can be checked that $\mathrm{D}_{\epsilon}$ is the only Riemann surface in $Y$ with boundary on $M_{\epsilon}$ preserved by a torus action of the form (2.26) with $n_{1} \neq 0$.

Next note that setting $\epsilon=0$ in the above construction yields a lagrangian cycle $M_{0}$ on the singular conifold $X_{0}$. In terms of the invariant monomials $(x, y, z, w)$, the defining equations of $M_{0}$ in $X_{0}$ are

$$
y-\bar{w}=0, \quad|x|-|z|=c .
$$

Since $c>0, x$ cannot vanish, hence $M_{0}$ is contained in the complement of the singular point $x=y=z=w=0$. Moreover, it is easy to check that $M_{0}$ is lagrangian with respect to the symplectic form $\omega_{0}$ obtained by restricting the standard symplectic form $\omega_{\mathbb{C}^{4}}$ to the complement of the singular point in $X_{0}$. Note also that equation (2.27) yields equation (2.15) at $c=0$, confirming that the present construction is a lifted version of the previous one.

The family of lagrangian cycles $L_{\mu} \subset X_{\mu}, \mu>0$ is defined by the same equations, (2.27), now interpreted as equations on the deformation $X_{\mu}$. It is straightforward to check that $L_{\mu}$ is lagrangian with respect to the symplectic form $\left.\omega_{\mathbb{C}^{4}}\right|_{X_{\mu}}$ and it is preserved by the torus action (2.20). The resulting transition between lagrangian cycles is schematically represented in figure (3).

Again, comparison with equation (2.12) shows that the cycle $L_{\mu}$ is a lift of the (inverse image of the) conormal bundle $\phi_{\mu}^{-1}\left(N_{K}^{*}\right)$. Moreover there is a unique torus invariant holomorphic cylinder $C_{\mu}$ in $X_{\mu}$ with one boundary component in $L_{\mu}$ and the second contained in the vanishing cycle $S_{\mu}$. This is obtained intersecting the two lagrangian cycles, $L_{\mu}, S_{\mu}$ with the holomorphic curve $C_{\mu} \subset X_{\mu}$ given by

$$
y=0, \quad x z=\mu .
$$




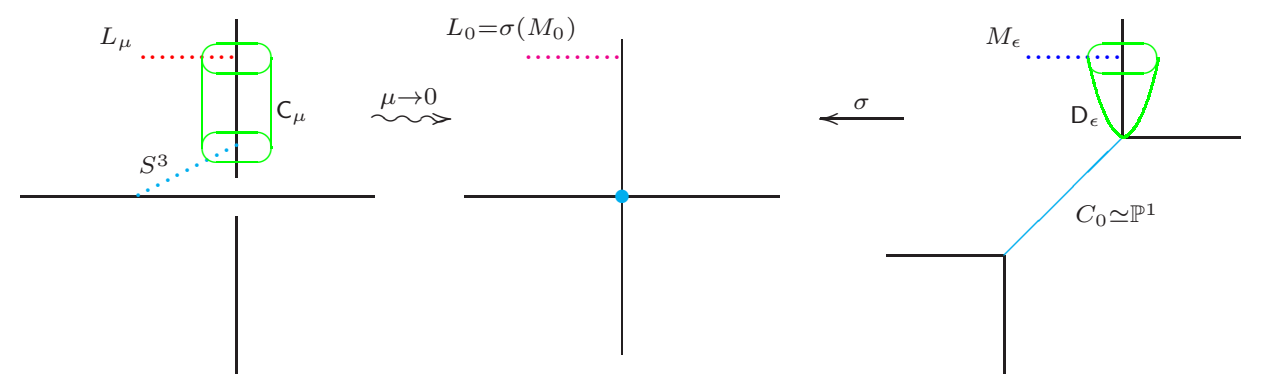

Figure 3. Conifold transition for toric lagrangian cycles.

One then finds two circles determined by the equations

$$
\begin{array}{ll}
C_{\mu} \cap L_{\mu}: & y=w=0, \quad x z=\mu, \quad|x|=\frac{c+\sqrt{c^{2}+4 \mu^{2}}}{2}, \\
C_{\mu} \cap S_{\mu}: & |x|=|z|=\sqrt{\mu}, \quad y=w=0 .
\end{array}
$$

The cylinder $C_{\mu}$ is given by

$$
y=w=0, \quad x z=\mu, \quad \sqrt{\mu} \leq|x| \leq \frac{c+\sqrt{c^{2}+4 \mu^{2}}}{2} .
$$

A different construction of lagrangian cycles for more general knots will be presented in the next section.

\section{Algebraic knots, Lagrangian CyCles And CONIFOld transitions}

The goal of this section is to present a construction of lagrangian cycles in $T^{*} S^{3}$ lifting the conormal bundle $N_{K}^{*}$ of any knot $K \subset S^{3}$. Note that such a construction was previously carried out in [21, where it was also proven that the resulting lagrangian cycles are related to totally real cycles on the resolved conifold via the conifold transition. Moerover, there is a well defined Gromov-Witten theory with boundary conditions on the totally real cycles, constructed in 21 via symplectic methods. As discussed in more detail below, the construction employed in this paper is a generalization of 21] motivated by the large $\mathrm{N}$ duality considerations explained in section (2.1). In particular, in this approach the lagrangian cycles associated to algebraic knots are naturally equipped with holomorphic cylinders with one boundary component in the lifted conormal bundle, the second boundary component being a knot in $S^{3}$ in the isotopy class of $K$. It will also be shown that these cycles are related by the conifold transition to lagrangian cycles in the small resolution of the conifold. For $K$ algebraic, the construction also yields a singular holomorphic discs $D_{\epsilon}$ in the resolved conifold with boundary on the corresponding lagrangian cycles. Furthermore, if $K$ is a torus knot, the resulting Gromov-Witten theory on the resolution with lagrangian boundary conditions turns out to be computable using a virtual localization approach similar to [18] and [23].

The notation and geometric set-up is as in the previous section. The total space of the cotangent bundle $T^{*} S^{3}$ is denoted by $X$ and will be identified with the subspace of $\mathbb{R}^{4} \times \mathbb{R}^{4}$ determined by equations (2.4). In this presentation, the canonical symplectic form $\omega_{X}$ is given by equation (2.5). The natural projection map $X \rightarrow S^{3}$ is denoted by $\pi$ and the zero section is denoted by $S$. 
3.1. Knots and lagrangian cycles in $T^{*} S^{3}$. Consider a smooth closed curve $\gamma: S^{1} \rightarrow X$ such that the projection $\pi \circ \gamma: S^{1} \rightarrow S^{3}$ is a smooth knot $K$ in $S^{3}$. In particular, $\gamma$ intersects each fiber of $X \rightarrow S^{3}$ at most once, otherwise its projection to $S^{3}$ would have self-intersection points. Suppose the map $\gamma$ is given by

$$
\theta \in S^{1} \rightarrow(\vec{u}, \vec{v})=(\vec{f}(\theta), \vec{g}(\theta)),
$$

where $\vec{f}(\theta)=\left(f_{j}(\theta)\right), \vec{g}(\theta)=\left(g_{j}(\theta)\right), j=1, \ldots, 4$, are smooth periodic functions of $\theta$.

The total space of the conormal bundle $N_{K}^{*}$ to $K$ in $S^{3}$ is defined by the equations

$$
\vec{u}=\vec{f}(\theta), \quad \dot{\vec{f}}(\theta) \cdot \vec{v}=0 .
$$

where $\dot{\vec{f}}(\theta)=d \vec{f}(\theta) / d \theta$. Then a straightforward computation yields

$$
\left.\omega_{X}\right|_{N_{K}}=\left.\left(d \sum_{j=1}^{4} v_{j} d u_{j}\right)\right|_{N_{K}}=d\left(\sum_{j=1}^{4} v_{j} \dot{f}_{j} d \theta\right)=0,
$$

confirming that $N_{K}^{*}$ is a lagrangian cycle in $X$.

Now consider the three-cycle $L_{\gamma} \subset T^{*} S^{3}$ determined by the equations

$$
\vec{u}=\vec{f}(\theta), \quad \dot{\vec{f}}(\theta) \cdot(\vec{v}-\vec{g}(\theta))=0 .
$$

By construction $L_{\gamma}$ is a cycle in the total space of the restriction $\left.T^{*} S^{3}\right|_{K}$. The restriction of the canonical projection $\pi: T^{*} S^{3} \rightarrow S^{3}$ yields a projection $\pi_{L_{\gamma}}$ : $L_{\gamma} \rightarrow K$. The fiber of $\pi_{L_{\gamma}}$ over a point $p \in K$ is the two plane in $T_{p}^{*} S^{3}$ determined by the second equation in (3.1), which is linear in $v_{j}$. Basically, $L_{\gamma}$ is obtained by a fiberwise translation of $N_{K}$ by a translation vector depending on the point $p \in K$. The restriction of the canonical symplectic form to $L_{\gamma}$ is given by

$$
\left.\omega_{X}\right|_{L_{\gamma}}=\left.\left(d \sum_{j=1}^{4} v_{j} d u_{j}\right)\right|_{L_{\gamma}}=d\left(\sum_{j=1}^{4} v_{j} \dot{f}_{j}(\theta) d \theta\right)
$$

Using the second equation in (3.1),

$$
\sum_{j=1}^{4} v_{j} \dot{f}_{j}(\theta) d \theta=\sum_{j=1}^{4} g_{j}(\theta) \dot{f}_{j}(\theta) d \theta
$$

on $L_{\gamma}$. Therefore

$$
\left.\omega_{X}\right|_{L_{\gamma}}=d\left(\sum_{j=1}^{4} g_{j}(\theta) \dot{f}_{j}(\theta) d \theta\right)=0 .
$$

In conclusion, $L_{\gamma}$ is a lagrangian cycle on $T^{*} S^{3}$. Note that the intersection of $L_{\gamma}$ with the zero section $\vec{v}=0$ is determined by the equations

$$
\vec{u}=\vec{f}(\theta), \quad \dot{\vec{f}}(\theta) \cdot \vec{g}(\theta)=0 .
$$

For sufficiently generic $\vec{f}(\theta), \vec{g}(\theta)$ this intersection will be empty, such that $L_{\gamma}$ is a lift of the conormal bundle $N_{K}^{*}$ off the zero section.

Note also that the lift constructed in [21] is a special case of the above construction obtained by setting $\vec{g}(\theta)=\dot{\vec{f}}(\theta)$. The main reason for the above generalization is that at least for algebraic cycles it also yields specific holomorphic open string instantons interpolating between the lifted conormal bundle and the vanishing cycle $S^{3}$ in the deformed conifold. This will be explained next. 
3.2. Lagrangian cycles for algebraic knots. So far this construction is fairly general and can be applied to any knot in $K \subset S^{3}$, for any lift $\gamma: S^{1} \rightarrow X$ satisfying the above conditions. In the special case when $K$ is an algebraic knot there is a preferred construction of the lift $\gamma$ motivated by AdS/CFT correspondence. The main idea is to obtain a one-cycle $\gamma$ as in section (3.1) by intersecting an $S^{2}$ bundle $P_{a} \subset T^{*} S^{3}$ of radius $a>0$ with the image $\phi_{\mu}\left(C_{\mu}\right)$ of a certain holomorphic curve $C_{\mu} \subset X_{\mu}$ associated to $K$ as explained below. Here $\phi_{\mu}: X_{\mu} \rightarrow X$ is the symplectomorphism given in equation (2.6).

Suppose $K$ is the link of the plane curve singularity $f(x, y)=0$ in $\mathbb{C}^{2}$. For simplicity assume that the curve $f(x, y)=0$ is irreducible and smooth away from $x=y=0$, and $K$ is connected. Consider the complete intersection $Z_{\mu} \subset X_{\mu}$ determined by

$$
f(x, y)=0, \quad f(z,-w)=0 .
$$

Suppose that $f(x, y)$ is sufficiently generic such that $Z_{\mu}$ is smooth for generic $\mu>0$. Note that $Z_{\mu}$ may have several distinct connected components even though the plane curve $f(x, y)=0$ is assumed irreducible. For example consider the case of torus knots, $f(x, y)=x^{r}-y^{s}$ with $(r, s)$ coprime positive integers. Then equations (3.2) imply

$$
(x z)^{r}-(-y w)^{s}=0,
$$

and substitution in the deformed conifold equation, $x z-y w=\mu$, yields

$$
(x z)^{r}-(\mu-x z)^{s}=0 .
$$

Therefore $x z=\eta$, where $\eta$ is a solution of the polynomial equation $t^{r}-(\mu-t)^{s}=0$. Each such solution $\eta$ determines a connected component of $Z_{\mu}$ of the form

$$
(x, y, z, w)=\left(t^{s}, t^{r}, \eta t^{-s},(\mu-\eta) t^{-r}\right)
$$

with $t \in \mathbb{C} \backslash\{0\}$.

Obviously, if $f(x, y)$ is a polynomial with real coefficients, $Z_{\mu}$ is preserved by the antiholomorphic involution (2.8). This will be assumed to be the case from now on. Then each connected component of the intersection of $Z_{\mu}$ with the fixed point locus $S_{\mu}=X_{\mu}^{\tau_{\mu}}$ is isomorphic to the one-cycle

$$
|x|^{2}+|y|^{2}=\mu, \quad f(x, y)=0
$$

in $\mathbb{C}^{2}$. For sufficiently small $\mu>0$, this is the link of the plane curve singularity $f(x, y)=0$ in $\mathbb{C}^{2}$. Note that the symplectomorphism $\phi_{\mu}$ maps $S_{\mu}$ to the zero section $S=\{\vec{v}=0\}$ in $X=T^{*} S^{3}$.

Now let $P_{a}=\{|\vec{v}|=a\}, a>0$, be the sphere bundle of radius $a$ in $X=T^{*} S^{3}$, and $B_{a} \subset X$ be the bounding disc bundle,

$$
B_{a}=\{(\vec{u}, \vec{v})|| \vec{v} \mid \leq a\} .
$$

Suppose there is a connected component $C_{\mu}$ of $Z_{\mu}$ with nontrivial intersection with the vanishing cycle $S_{\mu}$. As observed above each connected component of the intersection must be isomorphic to the link of the plane curve singularity $f(x, y)=$ 0 . Since $\phi_{\mu}\left(C_{\mu}\right)$ has nontrivial intersection with the zero section $S \subset X$, it will also intersect all sphere bundles $P_{a} \subset X$ for sufficiently small values of $a \in \mathbb{R}_{>0}$. In fact for sufficiently small $a>0$ the intersection $\phi_{\mu}\left(C_{\mu}\right) \cap B_{a}$ will be foliated by disjoint connected one-cycles $\gamma_{\mu, a^{\prime}}=\phi_{\mu}\left(C_{\mu}\right) \cap P_{a^{\prime}}, 0 \leq a^{\prime} \leq a$. Then applying the construction in section (3.1) to $\gamma_{\mu, a}$ yields a lagrangian cycle $L_{\gamma_{\mu, a}} \subset X$. The inverse image $L_{\mu, a} \subset X_{\mu}=\phi_{\mu}^{-1}\left(L_{\gamma_{\mu, a}}\right)$ is a lagrangian cycle in $X_{\mu}$ intersecting $C_{\mu}$ 
along the one-cycle $\phi_{\mu}^{-1}\left(\gamma_{\mu, a}\right)$. Moreover, by construction there is a holomorphic cylinder in $\mathrm{C}_{\mu, a} \subset X_{\mu}$ contained in $C_{\mu}$, with one boundary component in $S_{\mu}$ and the second boundary component in $L_{\mu, a}$. This is precisely the basic set-up of large $N$ duality in terms of lifted lagrangian cycles described in section (2.1), above equation (2.19). In order to keep the notation simple $L_{\mu, a}, \mathrm{C}_{\mu, a}$ will be simply denoted by $L_{\mu}, \mathrm{C}_{\mu}$ the $a$-dependence being implicitly understood.

Two important questions must be addressed at this point. The first issue is whether the holomorphic cylinder $\mathrm{C}_{\mu}$ with lagrangian boundary conditions on $S_{\mu}$, $L_{\mu}$ is unique and rigid, at least up to a torus action. This question will be answered affirmatively for torus knots in section (6), being left open at the moment for more general algebraic knots.

The second problem is whether one can construct a family of lagrangian cycles $M_{\epsilon}$ on $Y$ completing the geometric transition picture represented in (2.17). This will be shown to be the case for any algebraic knot in the next subsection, with the caveat that the resulting Gromov-Witten theory with lagrangian boundary conditions on $M_{\epsilon}$ is again tractable only for torus knots.

A first step towards completing the diagram (2.17) is to understand the specialization of the above construction at $\mu=0$. The specialization of $Z_{\mu}$ is a reducible curve $Z_{0}$ in the singular conifold $X_{0}$ with at least two irreducible components $C^{ \pm}$ given by

$$
f(x, y)=0, \quad z=w=0,
$$

respectively

$$
f(z,-w)=0, \quad x=y=0 .
$$

These components meet at the conifold singularity, which is also a singular point of $Z_{0}$. Since $f(x, y)$ is assumed real, the antiholomorphic involution $\tau_{0}: X_{0} \rightarrow X_{0}$ exchanges $C^{ \pm}$.

For concreteness, consider again the example of torus knots, $f(x, y)=x^{r}-y^{s}$. In this case the defining equations of $Z_{0}$ imply that $t=x z$ must be a solution of the polynomial equation $t^{r}-(-t)^{s}=0$. Therefore $x z=0$ or $x z=\eta$ with $\eta^{r-s}=$ $(-1)^{s+1}$. This implies that $Z_{0}$ has $r-s+1$ connected components. The connected component corresponding to $x z=0$ is the union of the two irreducible components $C^{ \pm}$defined above, which intersect at the singular point $x=y=z=w=0$. Each connected component corresponding to $x z=\eta$ is determined by the equations

$$
x z=y w=\eta, \quad x^{r}=y^{s} .
$$

Since these equations are invariant under the $\mathbb{C}^{\times}$-action

$$
(x, y, z, w) \mapsto\left(\alpha^{s} x, \alpha^{r} y, \alpha^{-s} z, \alpha^{-r} w\right)
$$

and $x, y, z, w$ cannot vanish, each such component is isomorphic to $\mathbb{C}^{\times}$.

Returning to the general case, let $\gamma^{ \pm}$be the one-cycles obtained by intersecting the inverse images $\phi_{0}\left(C^{ \pm} \backslash\{0\}\right)$ with the sphere bundle $P_{a}$. It is straightforward to check that $\tau_{0}$ exchanges the image cycles $\phi_{0}\left(\gamma^{ \pm}\right)$. Applying the construction of section (3.1) to the cycle $\gamma^{+}$, one obtains a lagrangian cycle $L_{\gamma^{+}}$in $X$. The inverse image $L_{0}=\phi_{0}^{-1}\left(L_{\gamma^{+}}\right)$is a lagrangian cycle in $X_{0}$. For sufficiently small $\mu \in \mathbb{R}_{>0}$ there exists an irreducible component $C_{\mu}$ of $Z_{\mu}$ such that the intersection $\phi_{\mu}\left(C_{\mu}\right) \cap P_{a}$ has a connected component $\gamma_{\mu}$ which specializes to $\gamma^{+}$at $\mu=0$. The resulting family of lifted lagrangian cycles $L_{\mu}$ specializes to $L_{0}$ at $\mu=0$. 
This completes the bottom part of diagram (2.17). The remaining part will be constructed in the next subsection.

3.3. Lagrangian cycles in the resolved conifold. Recall the resolved conifold $Y$ is determined by equations (2.2) in $\mathbb{C}^{4} \times \mathbb{P}^{1}$ and $\sigma: Y \rightarrow X_{0}$ denotes the natural contraction map to the singular conifold. The family of symplectic manifolds $Y_{\epsilon}$ in diagram (2.17) is determined by the symplectic forms

$$
\omega_{Y, \epsilon}=\left.\left(\omega_{\mathbb{C}^{4}}+\epsilon^{2} \omega_{\mathbb{P}^{1}}\right)\right|_{Y}
$$

where $\omega_{\mathbb{C}^{4}}$ is the standard symplectic form on $\mathbb{C}^{4}$ and $\omega_{\mathbb{P}^{1}}$ is the Fubini-Study form on $\mathbb{P}^{1}$.

The family of lagrangian cycles $M_{\epsilon} \subset Y_{\epsilon}$ will be constructed using 31, Lemm. 7.11], which provides a geometric relation between the symplectic structures on $Y_{\epsilon}$, $X_{0}$. First it will be helpful to recall the statement of [31, Lemm. 7.11] for the one-point blow-up $\eta: \widetilde{\mathbb{C}}^{2} \rightarrow \mathbb{C}^{2}$ at the origin. Consider the following one parameter family of symplectic forms on the blow-up

$$
\omega_{\widetilde{\mathbb{C}}^{2}, \epsilon}=\left.\left(\omega_{\mathbb{C}^{2}}+\epsilon^{2} \omega_{\mathbb{P}^{1}}\right)\right|_{\widetilde{\mathbb{C}}^{2}} .
$$

For any $\epsilon \in \mathbb{R}_{>0}$ let $B(\epsilon) \subset \mathbb{C}^{2}$ be the ball $|z|^{2}+|y|^{2} \leq \epsilon^{2}$ and $\widetilde{B}(\epsilon)=\eta^{-1}(B(\epsilon))$ be its inverse image in $\widetilde{\mathbb{C}}^{2}$. Note that there is a radial map $\rho_{\epsilon}: \mathbb{C}^{2} \backslash\{0\} \rightarrow \mathbb{C}^{2} \backslash B(\epsilon)$,

$$
\rho_{\epsilon}(y, z)=\frac{\sqrt{|z|^{2}+|y|^{2}+\epsilon^{2}}}{\sqrt{|z|^{2}+|y|^{2}}}(y, z)
$$

Then [31, Lemm. 7.11] proves that the map $\psi_{\epsilon}: \widetilde{\mathbb{C}}^{2} \backslash E \rightarrow \mathbb{C}^{2} \backslash B(\epsilon)$,

$$
\psi_{\epsilon}=\left.\rho_{\epsilon} \circ \eta\right|_{\widetilde{\mathbb{C}}^{2} \backslash E}
$$

is a symplectomorphism for any $\epsilon \in \mathbb{R}_{>0}$, where $E \subset \widetilde{\mathbb{C}}^{2}$ denotes the exceptional curve.

In order to apply [31, Lemm. 7.11] to the present situation, note that $Y$ can be regarded as the quadric hypersurface $x \lambda=w \rho$, in the fourfold $Z$ determined by $z \rho=y \lambda$ in $\mathbb{C}^{4} \times \mathbb{P}^{1}$. Obviously, $Z \simeq \mathbb{C}^{2} \times \widetilde{\mathbb{C}}^{2}$ where $\widetilde{\mathbb{C}}^{2}$ is the one point blow-up of $\mathbb{C}^{2}$ at the origin. Next note that the map $\varrho_{\epsilon}=1_{\mathbb{C}^{2}} \times \rho_{\epsilon}: \mathbb{C}^{2} \times\left(\mathbb{C}^{2} \backslash\{0\}\right) \rightarrow$ $\mathbb{C}^{2} \times\left(\mathbb{C}^{2} \backslash B(\epsilon)\right)$ preserves the nodal threefold $X_{0} \subset \mathbb{C}^{2} \times \mathbb{C}^{2}$, mapping $X_{0} \backslash\{0\}$ to the open subset $X_{0}(\epsilon)=X_{0} \backslash X_{0} \cap\left(\mathbb{C}^{2} \times B(\epsilon)\right)$. Note also that the exceptional $(-1,-1)$ curve $C_{0} \subset Y$ coincides with the curve $\{0\} \times E \subset \mathbb{C}^{2} \times \widetilde{\mathbb{C}}^{2}$. This implies that the complement of the zero section $Y \backslash C_{0}$ coincides with the open subset $Y \cap \mathbb{C}^{2} \times\left(\widetilde{\mathbb{C}}^{2} \backslash E\right)$. Then the map

$$
\phi_{\epsilon}: Y \backslash C_{0} \rightarrow X_{0}(\epsilon), \quad \phi_{\epsilon}=\left.\left(\varrho_{\epsilon} \circ \sigma\right)\right|_{Y \backslash C_{0}} .
$$

is a symplectomorphism.

Returning to the construction of the lagrangian cycle $M_{\epsilon} \subset Y_{\epsilon}$, recall that the family of complete intersection curves $C_{\mu} \subset X_{\mu}$ given by

$$
f(x, y)=0, \quad f(z,-w)=0
$$

specializes to a reducible curve at $\mu=0$ with two components

$$
\begin{array}{ll}
C^{+}: & f(x, y)=0, \quad z=w=0 \\
C^{-}: & f(z,-w)=0, \quad x=y=0 .
\end{array}
$$


The intersection of $\phi_{0}\left(C^{+}\right)$with the sphere bundle $P_{a}$ yields a one-cycle $\gamma^{+}$, the limit of the cycles $\gamma_{\mu}^{+}$as $\mu \rightarrow 0$. The corresponding lagrangian cycle $L_{\gamma^{+}}$is the limit of $L_{\mu}^{+}$as $\mu \rightarrow 0$.

Now consider the one-cycle

$$
\gamma_{\epsilon}^{+}=\phi_{0} \circ \varrho_{\epsilon} \circ \phi_{0}^{-1} \circ \gamma^{+}: S^{1} \rightarrow X
$$

on $X$ obtained by applying the radial map to the inverse image $\phi_{0}^{-1} \circ \gamma^{+}$of the path $\gamma^{+}$. Then set

$$
M_{\epsilon}=\phi_{\epsilon}^{-1}\left(\phi_{0}^{-1}\left(L_{\gamma_{\epsilon}^{+}}\right)\right)=\sigma^{-1}\left(\varrho_{\epsilon}^{-1}\left(\phi_{0}^{-1}\left(L_{\epsilon}\right)\right)\right),
$$

where $L_{\gamma_{\epsilon}^{+}} \subset X$ is the lagrangian cycle obtained by applying the construction of section (3.1) to $\gamma_{\epsilon}^{+}$. By construction $L_{\gamma_{\epsilon}^{+}}$intersects the dilation $\left.\varrho_{\epsilon}\left(\phi_{0}\left(C^{+}\right)\right)\right)$of the curve $\phi_{0}\left(C^{+}\right)$along the cycle $\gamma_{\epsilon}^{+}$. Therefore the inverse image $\varrho_{\epsilon}^{-1}\left(\phi_{0}^{-1}\left(L_{\gamma_{\epsilon}^{+}}\right)\right)$ intersects the plane curve $C^{+} \subset X_{0}$ along the cycle $\phi_{0}^{-1} \circ \gamma_{\epsilon}^{+}$. Since $\sigma: Y \backslash C_{0} \rightarrow$ $X_{0} \backslash\{0\}$ is an isomorphism of complex manifolds, it follows that $M_{\epsilon}$ intersects the strict transform $C \subset Y$ of $C^{+}$along the cycle $\eta_{\epsilon}=\sigma^{-1} \circ \phi_{0}^{-1} \circ \gamma_{\epsilon}^{+}$. The strict transform $C$ is the plane singular curve cut by the equations

$$
f(x, y)=0, \quad \lambda=0
$$

on $Y$. Therefore it is a singular plane curve isomorphic to $C^{+}$, contained in the fiber $\lambda=0$ of $Y$ over $\mathbb{P}^{1}$. The singular point $p \in C$ is the unique point of intersection with the zero section, $x=y=0, \lambda=0$. The cycle $\eta_{\epsilon}$ divides $C$ into two connected components, the component containing $p$ being a singular holomorphic disc $\mathrm{D}_{\epsilon}$ in $Y_{\epsilon}$ with boundary $\eta_{\epsilon} \subset M_{\epsilon}$. This is precisely the geometric set-up outlined in diagram (2.17). In order to obtain a complete large $N$ duality picture, one should prove that the holomorphic disc $\mathrm{D}_{\epsilon}$ is rigid, which is a difficult technical question for general algebraic knots. Section (6) will provide an affirmative answer for torus knots, leaving the general case for future work.

Assuming that $D_{\epsilon}$ is rigid, the next problem is the computation of its multicover contributions to the Gromov-Witten theory with lagrangian boundary conditions on $M_{\epsilon}$. One angle on this problem is to try to generalize the computations of [18] based on stable maps with lagrangian boundary conditions to the present case. This approach requires a torus action preserving $M_{\epsilon}, \mathrm{D}_{\epsilon}$, which is the case only for torus knots. In this case, the details of the virtual localization computation are presented in section (6), the resulting formulas being in agreement with large $N$ duality predictions.

A second approach follows from string duality considerations as in [12, 34, converting the calculation of of topological open A-model amplitudes to D-brane bound state counting. In this framework, the topological amplitudes are expressed in terms of BPS states as in Donaldson-Thomas type invariants, making a direct connection with the 32. This will be discussed next.

\section{D-brane bound states and the Hilbert scheme}

The goal of this section is to provide a physical explanation for the work of Oblomkov and Shende [32] on plane curve singularities in the framework of large $N$ duality. The geometric set-up will be the same as in section (3.3), namely a lagrangian cycle $M_{\epsilon} \subset Y_{\epsilon}$ intersecting a singular plane curve $C \subset Y$ along a 
smooth connected one-cycle $\eta: S^{1} \rightarrow M_{\epsilon}$. The curve $C$ is given by

$$
f(x, y)=0, \quad \lambda=0,
$$

on $Y$ and it will be assumed that it has only one singular point $p$, given by $x=y=0$, $\lambda=0$. The cycle $\eta_{\epsilon}$ divides $C$ into two connected components, the component containing $p$ being a holomorphic disc $\mathrm{D}_{\epsilon}$ with boundary on $M_{\epsilon}$. Note that $M_{\epsilon} \simeq$ $\mathbb{R}^{2} \times S^{1}$ and the cycle $\eta_{\epsilon}$ is a generator of $H_{1}\left(M_{\epsilon}\right) \simeq \mathbb{Z}$. It will be assumed that $\mathrm{D}_{\epsilon}$ is rigid, which is in fact proven in section (6) for curves of the form $f(x, y)=x^{r}-y^{s}$. The subscript $\epsilon$ will be dropped in this section because all considerations below hold for any fixed arbitrary value of $\epsilon>0$.

According to cite [34, 22, 29, string duality transformations show that open topological string amplitudes with lagrangian boundary conditions on $M$ are determined by counting supersymmetric M2-brane or D2-D0 bound states in different duality frames. This is achieved by studying the low-enery effective action for type IIA D4-brane wrapped on the lagrangian cycle $M$, resulting in a string-like object in the four transverse dimensions. Open topological string amplitudes with boundary conditions on $M$ determine certain holomorphic couplings in the low energy effective action of this string. Since $H_{1}(M) \simeq \mathbb{Z}$ is generated by $\eta$, open string instantons with fixed genus $g \in \mathbb{Z}_{>0}$ and $h=1$ boundary components are topologically classified by the wrapping number $d \in \mathbb{Z}_{>0}$ on the holomorphic curve $C_{0}$ and the winding number $k \in \mathbb{Z}_{\geq 1}$ about the cycle $\eta$. The corresponding Gromov-Witten invariants with lagrangian boundary conditions will be denoted by $G W_{g, 1}(d, k)$. Only topological open string amplitudes with winding number $k=1$ will be considered in the following, because we are interested in the Wilson loop observables in the fundamental representation. Moreover we can assume, with no loss of generality that we have only 1 spectator lagrangian A-brane and we replace $\operatorname{Tr} V$ with $V$. According to 34, these amplitudes determine terms of the form

$$
\int d^{4} x d^{4} \theta \delta^{(2)}(x) \delta^{(2)}(\theta) F_{g, 1}(t, V)\left(W^{2}\right)^{g}
$$

in the effective action of the string, where

$$
F_{g, 1}(t, V)=\sum_{d \geq 0} g_{s}^{2 g-1} e^{-d t} G W_{g, 1}(d, 1) V
$$

Here $W_{\alpha \beta}$, where $\alpha, \beta$ are symmetric spinor indices, denotes the four dimensional graviphoton multiplet, and $t$ denotes the vector multiplet whose top component is the Kähler modulus of the zero section $C_{0} \subset Y$. As in section (2.1), $V$ is the holonomy of a background flat $U(1)$ gauge field on the D4-brane. The four dimensional superspace integral is restricted to the string world-sheet by the $\delta$ functions $\delta^{(2)}(x), \delta^{(2)}(\theta)$.

The M-theory lift of this configuration is an M5-brane wrapping the same lagrangian cycle $M$. Holomorphic IIA world-sheet instantons lift to open M2-branes with boundary on $M$ wrapping the disc $\mathrm{D}$ and the zero section $C_{0}$. The low-energy effective theory of the M5-brane is now a three dimensional theory containing a spectrum of supersymmetric particles corresponding to bound states of open M2branes. The low energy degrees of freedom include a three-dimensional $N=2$ $U(1)$ vector multiplet, the reduction of the M5-brane self-dual tensor multiplet on a harmonic generator of $H^{1}\left(M_{\epsilon}\right) \simeq \mathbb{Z}$. In addition the space-time effective action includes a $U(1)$ gauge field field in the supergravity multiplet. The three-dimensional 
BPS particles carry integer charges $(k, d) \in \mathbb{Z}$ with respect to these gauge fields. Geometrically, $k, d$ are the M2-brane multiplicity on the disc D, respectively the zero section $C_{0}$. The five dimensional $S O(4)$ little group is broken to $U(1) \times U(1)$ by the M5-brane, the first factor being the little group in the M5 three dimensional effective theory. The second factor is generated by rotations in the two transverse dimensions. Therefore the BPS spectrum is graded by two spin quantum numbers $\sigma, j \in \mathbb{Z}+1 / 2$. The degeneracies of BPS states will be denoted accordingly by $N_{k, d, \sigma, j}$. By analogy with [12] a Schwinger computation shows that the couplings $F_{g, 1}(t, V)$ are given by

$$
F_{g, 1}(t, V)=\sum_{d \geq 0} \sum_{\sigma \in \mathbb{Z}} \frac{N_{d, \sigma}}{2 \sin \left(g_{s} / 2\right)} e^{-d t+i \sigma g_{s}} V
$$

where $N_{d, \sigma}$ is a BPS index given by

$$
N_{d, \sigma}=\sum_{j \in \mathbb{Z}+1 / 2}(-1)^{2 j+1} N_{1, d, \sigma, j} .
$$

In particular note that the coefficients $N_{d, \sigma}$ have to be integral but not necessarily positive.

The above expression is the restriction of 34, Eqn. 4.4] to open string amplitudes of winding number 1 , therefore the sum over the integer $n \geq 1$ corresponding to degree $n$ multicover contributions collapses to a single term, $n=1$. A more convenient form of equation (4.1) can be obtained by a change of variables $q=e^{i g_{s}}$, $Q=e^{-t}$, and a redefinition of the spin quantum number, $\sigma=s+1 / 2, s \in \mathbb{Z}$. Then equation (4.1) becomes

$$
F_{g, 1}(t, V)=\sum_{d \geq 0} \sum_{\sigma \in \mathbb{Z}} \frac{N_{d, s} q^{s} Q^{d}}{1-q} V .
$$

In order to compute the BPS numbers $N_{d, s}$, one has to count spin $s+1 / 2$ bound states of an open M2-brane wrapping the singular holomorphic disc D and $d$ M2branes wrapping the zero section $C_{0}$. Note that using the Large $\mathrm{N}$ duality this implies that the expectation value of the Wilson loop in the fundamental representation of the knot, which is known as the HOMFLY polynomial of the knot, is given by this expression (recalling that $\operatorname{Tr} U$ is paired up with $\operatorname{Tr} V$ ):

$$
\langle T r U\rangle=\sum_{d \geq 0} \sum_{\sigma \in \mathbb{Z}} \frac{N_{d, s} q^{s} Q^{d}}{1-q}
$$

It is known [13, 12 that $d$ M2-branes wrapping the compact curve $C_{0}$ form supersymmetric bound states only if $d=1$, in which case there is a single spin 0 state. Therefore the main problem is to understand bound state counting for open M2-branes wrapping the singular disc D. This is most efficiently done reducing the problem to counting D2-D0 bound states in a suitable weakly coupled Type IIA limit. More precisely, one can choose the M-theory circle such that that the M5-brane is mapped again to a D4-brane, but the M2-branes yield open D2-branes with boundary on the D4-brane. Furthermore the $d=0$ truncation of the right hand side of equation (4.2) is interpreted as a partition function of the form

$$
\sum_{n \geq 0} C_{n} q^{n}
$$


counting supersymmetric states of open D2-branes wrapping D bound to an arbitrary number $n$ of D0-branes [12, 19]. The coefficients $C_{n}$ in equation (4.4) are BPS indices counting states weighted by a sign determined by their spin. This index can be exactly computed in the semiclassical limit, in which case $C_{n}$ equals the Euler character of the moduli space of supersymmetric D-brane configurations. In order to understand the structure of such moduli spaces, it is helpful to consider first configurations of $n$ D0-brane bound to a D2-brane wrapping a fixed compact holomorphic curve $Z$ in some Calabi-Yau threefold. According to [19, 20, 35, such configurations are mathematically modeled by an abelian vortex configuration of degree $n$ on $Z$. The basics of this formalism will be reviewed in some detail below.

4.1. D2-D0 bound states, vortices, and stable pairs. A degree $n$ abelian vortex is a triple pair $(\mathcal{L}, A, s)$ where $\mathcal{L}$ is a complex line bundle on $Z$ with first Chern class $c_{1}(\mathcal{L})=n, A$ is a $U(1)$ connection on $\mathcal{L}$ and $s$ is a section of $\mathcal{L}$ satisfying $D_{A} s=0$. This naturally captures the dates of the choice of the gauge field on the D2 brane, as well as the geometry of D0 brane, which can be identified with $s$. It corresponds to the bifundamental field charged under the D2-brane $U(1)$ stretched between D0-brane and D2-brane. The moduli space of triples $(\mathcal{L}, A, s)$ modulo unitary gauge transformations is isomorphic to the moduli space of pairs $(\mathcal{L}, s)$ modulo complexified gauge transformations, where $\mathcal{L}$ is a holomorphic line bundle on $Z$ and $s \in H^{0}(Z, \mathcal{L})$ is a nontrivial holomorphic section. The relation between differential geometric and algebraic geometric data follows as usual observing that any connection $A$ on a $C^{\infty}$ complex line bundle $\mathcal{L}$ determines a Dolbeault operator $\bar{\partial}_{A}$.

In the algebraic formulation, note that the zero locus of $s$ is a degree $n$ effective divisor $(s)$ on $Z$, that is a formal linear combination of points $\sum_{i=1}^{k} n_{i} p_{i}$ where $n_{i} \geq 1$ and $\sum_{i=1}^{k} n_{i}=n$. The points $p_{1}, \ldots, p_{k}$ represent the locations of the D0-branes and the integers $n_{i} \geq 1, i=1, \ldots, k$ the D0-brane multiplicity at each point. Assigning to each pair $(\mathcal{L}, s)$ the divisor $(s)$ yields an isomorphism between the moduli space of isomorphism classes of pairs $(\mathcal{L}, s)$ and the symmetric product $S^{n}(Z)=Z^{n} / \mathcal{S}_{n}$, where $\mathcal{S}_{n}$ is the permutation group on letters.

From the sheaf theoretic point of view, a pair $(\mathcal{L}, s)$ can be uniquely characterized up to gauge transformations by specifying the germs of local holomorphic sections of $\mathcal{L}$ near each point $p$ of $Z$. The simplest case is the trivial vortex configuration, when $\mathcal{L}$ is isomorphic to the trivial line bundle $\mathcal{O}_{Z}$ on $Z$ and $s$ is constant. The germs of local sections of $\mathcal{O}_{Z}$ near each point $p \in Z$ are simply germs of local holomorphic functions with no restrictions on the vanishing order at $p$. For a configuration $(\mathcal{L}, s)$ with $n>0$ the same holds locally near any point $p \in Z, p \neq p_{i}, i=1, \ldots, k$. Near one of the points $p_{i}$, the germs of holomorphic sections of $\mathcal{L}$ are identified with germs of meromorphic functions with at most a pole of order $n_{i}$ at $p_{i}$. In this local picture the section $s$ corresponds to the natural inclusion of the local sections of $\mathcal{O}_{Z}$ in the set of local sections of $\mathcal{L}$. Note that the complement is a finite dimensional vector space of dimension $n_{i}$. In terms of a local coordinate $z_{i}$ centered at $p_{i}$, this vector space is generated by sections of the form $\left\{z_{i}^{-l}\right\}, l=1, \ldots, n_{i}$. More abstractly, the local sections of $\mathcal{L}$ near $p_{i}$ form a rank 1 module over the local ring of functions $\mathcal{O}_{Z}$ generated by $\left\{z_{i}^{-n_{i}}\right\}$.

It may be also helpful to note that there is a dual mathematical model for D2-D0 configurations. In the dual model, $n_{i}$ D0-branes located at $p_{i}$ are described by the set of local holomorphic functions which vanish at least to order $n_{i}$ at $p_{i}$. This set 
is the ideal generated by $z_{i}^{n_{i}}$ in the ring of local functions near $p_{i}$. The geometric object characterized by this local behavior is the dual line bundle $\mathcal{L}^{-1}$, which is a sub-sheaf of the trivial line bundle $\mathcal{O}_{Z}$. In more abstract language, $\mathcal{L}^{-1} \subset \mathcal{O}_{Z}$ is the defining ideal sheaf of the effective divisor $(s)=\sum_{i=1}^{k} n_{i} p_{i}$.

Similar considerations apply [35, 36, to a singular curve $Z$, abelian vortices being generalized to stable pairs. This essentially means that one has to allow the gauge field $A$ to develop singularities at the singular points of the curve $Z$. While a complete analytic treatment of such singularities would be quite difficult, the sheaf theoretic point of view discussed above leads to an efficient construction of the moduli space. A single D0-brane supported at a smooth point $p$ was previously identified with the module of local meromorphic functions with at most a simple pole at $p$. If $p$ is a singular point of $Z$, a single D0-brane at $p$ is still defined by a module of local meromorphic functions, but this module may have more than one generator. Conceptually, this may be easily understood employing the dual model. Consider for example the plane curve singularity $x^{3}=y^{2}$. A D0-brane with multiplicity 1 located at the singular point $x=y=0$ cannot be described as the zero locus of a single local holomorphic function. If one simply sets $x=0$ or $y=0$ the defining equation of the curve reduces to $y^{2}=0$, respectively $x^{3}=0$. According to the previous paragraph this is in fact a D0-brane configuration with multiplicity 2 , respectively 3. A single D0-brane is the zero locus of two local functions, $(x, y)$ which generate an ideal in the ring of local holomorphic functions. The dual stable pair is given by a local module over the ring of local functions generated by two elements.

Informally, the main idea of this construction is that at a singular point $p$ of $Z$ the rank of the Chan-Paton line bundle $\mathcal{L}$ on $Z$ is allowed to jump in a controlled way, depending on the analytic type of the singularity at $p$. Effectively, the single D2brane on $Z$ behaves locally at $p$ as a stack of D2-branes with higher multiplicity $m \geq$ 1. For a fixed number of D0-branes $m$ may take finitely many values determined by $n$ and the singularity type. This point of view will be very useful in understanding bound state formation for D2-branes wrapping different holomorphic curves with transverse intersection.

A consequence of the above discussion is that the moduli space of D2-D0-brane configurations supported on $Z$ is no longer isomorphic to the symmetric product $S^{n}(Z)$. It has been shown in 36 that the moduli space of D2-D0-brane configurations supported on $Z$ is in this case isomorphic to the Hilbert scheme $\mathcal{H}^{n}(Z)$ of $n$ points on $Z$. The rigorous definition of the Hilbert scheme is not needed for the purpose of the present discussion, but it may be helpful to note that there is a natural map $\pi: \mathcal{H}^{n}(Z) \rightarrow S^{n}(Z)$ forgetting the extra algebraic structure associated to each singular point. From a physical point of view this means that the D0-branes are treated simply as non-interacting particles ignoring interactions due to open string effects.

Analogous considerations hold for D2-branes wrapping a smooth holomorphic disc $\mathrm{D}$ with boundary on a lagrangian cycle. The holomorphic line bundle $\mathcal{L}$ must now be equipped with a trivialization on the boundary of the disc $\partial \mathrm{D} \simeq S^{1}$, which is part of the boundary conditions on the D2-brane fields. Complex line bundles on the disc with boundary trivialization are topologically classified by the first Chern class, which takes values in the relative homology group $H_{2}(\mathrm{D}, \partial \mathrm{D}) \simeq \mathbb{Z}$. Moreover, since the section $s \in H^{0}(\mathcal{L})$ must be compatible with the trivialization, 
the number of zeroes of $s$, counted with multiplicity must equal the first Chern class $n$. Summing over all Chern classes yields the partition function

$$
\sum_{n \geq 0} C_{n} q^{n}=\sum_{n \geq 0} \chi\left(S^{n}(\mathrm{D})\right) q^{n}=\frac{1}{1-q}
$$

since the symmetric power $S^{n}(\mathrm{D})$ is contractible for any $n \geq 0$. Note that this result is the same as the winding number one partition function of a single lagrangian brane in $\mathbb{C}^{3}$ given [1] by the topological vertex $C_{\emptyset, \emptyset, \square}(q)$. As observed in [17, Sect 4], the above formula can be alternatively interpreted as the Hilbert series of the ring $\mathbb{C}[t]$ of polynomial functions on the complex line $\mathbb{C}$. By definition, the Hilbert series of a polynomial ring $R$ is

$$
H_{R}(q)=\sum_{n \geq 0} c_{n}(R) q^{n}
$$

where $c_{n}(R)$ is the number of degree $n$ monomials in $R$. Obviously, $H_{\mathbb{C}[t]}(q)$ is equal to the above partition function. It was explained in [17, Sect 4], that $H_{\mathbb{C}[t]}(q)$ can be also interpreted as a counting function of states in the Hilbert space $\mathcal{H}$ of a single quantum harmonic oscillator.

Next suppose $D$ has singular points away from the boundary. Without any loss of essential information, one may assume that D has only one singular point $p$. Several singular points may be treated analogously with no new conceptual issues.

In this case the Chern class of a singular vortex configuration admits a splitting, $n+l$, where $n \in \mathbb{Z}$ is determined as above by the trivialization on the boundary, and $l \in \mathbb{Z}$ is a contribution supported at the singular point. A rigorous account of this splitting is provided at the end of section (5), where it is also shown that $l$ takes finitely many values. In addition, one has to specify the multiplicity $m \geq 1$ of the singular vortex at $p$, as discussed above. Therefore the partition function will be in general of the form

$$
\sum_{l \geq 0} \sum_{m \geq 1} f_{l, m}(q)
$$

where only finitely many terms are nontrivial. Note that each term $f_{l, m}(q)$ is a power series in $q$ because for fixed values of $(l, m)$ one has to sum over all possible boundary trivializations, as in the smooth case. More detailed information on the terms $f_{l, m}(q)$ requires a more involved technical analysis, as shown for specific examples in section (4.3). A more immediate task at this stage is however to explain how the above general reasoning can be applied to more general M2-brane configurations supported on intersecting curves.

4.2. Intersecting M2-brane bound states. The relevant intersecting curve configurations for large $N$ duality consist of a singular holomorphic disc $\mathrm{D}$ as above meeting a smooth $(-1,-1)$ rational curve $C_{0}$ at the singular point $p$. One then has to count bound states of $k=1$ open M2-branes wrapping $\mathrm{D}$ and $d$ closed M2-branes wrapping the zero section $C_{0}$. As shown in [13, 12, M2-branes wrapping a $(-1,-1)$ curve $C_{0}$ with multiplicity $d \geq 1$ form bound states only for $d=1$, in which case the spectrum consists of one BPS state of spin 0 .

In addition, when an M2-brane wrapping $\mathrm{D}$ is added to the system one can form new bound states binding a membrane wrapping $C_{0}$ to the membrane wrapping $\mathrm{D}$. If $\mathrm{D}$ were smooth, the intersection between the two M2-branes would be modeled by 
a curve with a simple nodal singularity $x y=0$. This configuration can be viewed as a limit of a single M2-brane wrapping the smooth curve $x y=\epsilon$ as $\epsilon \rightarrow 0$. Therefore two intersecting M2-branes form in this case a single bound state.

However in the case of interest here $\mathrm{D}$ is a singular disk, which has local multiplicity $m \geq 1$ at the singular point $p$, even though its generic multiplicity 1 . In other words $m$ counts the number of 'points' at $p$. What this means is that if we were to consider an annulus which ends on one end on the D2-D0 brane bound state on one side, and on a transverse D-brane intersecting the curve at $p$ on the other, $m$ counts the Witten index for it. Therefore a membrane wrapping $C_{0}$ may bind to the singular membrane in $m$ distinct ways, depending on which local branch it is attached to. This results into a spectrum of $m$ BPS states in the low energy effective action. More generally, $d$ membranes wrapping $C_{0}$ can bind in $\left(\begin{array}{c}m \\ d\end{array}\right)$ distinct ways to the singular open membrane, resulting in as many BPS particles. In particular, if $d>m$ no irreducible bound state may be constructed. Therefore the partition function for such configurations must take the general form

$$
\sum_{l \geq 0} \sum_{m \geq 1} \sum_{d=0}^{m}\left(\begin{array}{c}
m \\
d
\end{array}\right)(-Q)^{d} f_{l, m}(q)=\sum_{m \geq 1} f_{m}(q)(1-Q)^{m}
$$

where

$$
f_{m}(q)=\sum_{l \geq 0} f_{l, m}(q)
$$

Here we used the fact, already seen for the unknot, that the fermion parity of the M2 brane wrapping $\mathbf{P}^{1}$ is -1 leading to $-Q$ for each such state in the above formula. Moreover, equation (4.1) predicts that

$$
f_{m}(q)=\frac{g_{m}(q)}{1-q}
$$

with $g_{m}(q)$ a polynomial with integral coefficients. These predictions will be confirmed by explicit computations for plane curves of the form

$$
x^{r}-y^{s}=0
$$

in the next section.

For completeness, it is worth noting that the combinatorial factors $\left(\begin{array}{c}m \\ d\end{array}\right)$ admit a geometric interpretation in the a weakly coupled IIA limit mapping M2-branes to D2-branes. Then the massless spectrum of open string stretching between a D2-brane on D and $d$ D2-branes on $C_{0}$ consists of an $N=2, d=4$ hypermultiplet reduced to one dimension. The bosonic components are two complex scalar fields $\phi, \psi$ transforming in the bifundamental representation of the D-brane gauge fields and its dual. Again, the singular D2-brane has effectively multiplicity $m$ at the singular point even if it is generically of rank one. Therefore $\phi, \psi$ may be identified with linear maps $\phi: \mathbb{C}^{m} \rightarrow \mathbb{C}^{n}, \psi: \mathbb{C}^{n} \rightarrow \mathbb{C}^{m}$ respectively. Then the F-term equations are simply

$$
\psi \circ \phi=0, \quad \phi \circ \psi=0 .
$$

This implies that the moduli space of flat directions modulo gauge transformations is isomorphic to a moduli space of representations of a quiver of the form

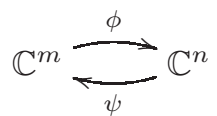


subject to the F-term equations. The stability conditions are determined as usual by the D-term equations,

$$
|\phi|^{2}-|\psi|^{2}=\xi
$$

The subtle aspect here is that even though the singular D2-brane has multiplicity $m$ at $p$, one should only mod out by $U(1) \times U(d)$ gauge transformations since the brane has generic of rank 1 . Moreover, since the diagonal $U(1)$ subgroup acts trivially on $\phi, \psi$, it suffices to mod out by $U(d)$ gauge transformations.

A straightforward analysis of the resulting stability condition shows that $\phi=0$ and $\psi$ must be surjective if $\xi<0$ and $\psi=0$ and $\phi$ must be surjective for $\xi>$ 0 . Therefore if $\xi>0$, the moduli space of stable representations modulo $U(d)$ gauge transformations is isomorphic to the grassmannian $G(m, d)$ of $d$-dimensional quotients of $\mathbb{C}^{m}$ if $d \leq m$ and empty if $d>m$. If $\xi<0$ the moduli space is just a point if $d \geq m$ and empty if $d<m$.

In string theory FI term $\xi$ is determined by the expectation value of the background fields on $Y$, such as the metric and B-field. The previous paragraph implies that for any choice of background fields such that $\xi>0$ the weakly coupled IIA analysis agrees with M-theory considerations. Namely, the moduli space of expectation values of open string modes is isomorphic to the grassmannian $G(m, d)$ which has Euler character $\left(\begin{array}{c}m \\ d\end{array}\right)$. This is precisely the number of bound states predicted by M-theory arguments.

4.3. Curves of type $(r, s)$. Returning to the setup described at the beginning of this section, consider a singular curve $C$ of the form

$$
x^{r}-y^{s}=0, \quad \lambda=0 .
$$

in a resolved conifold $Y$. Here $(r, s)$ are coprime positive integers and it will be assumed that $r>s \geq 1$. Note that $C$ has only one singular point $p$ given by $x=y=0, \lambda=0$. The construction of section (3.3) produces a lagrangian cycle $M \subset Y$ which intersects $C$ along a smooth connected one cycle $\eta$. Therefore $C$ is divided into two connected components, the holomorphic disc D being the component containing the singular point $p$. Note that $\mathrm{D}$ is preserved by the circle action

$$
(x, y, \zeta) \mapsto\left(e^{-i s \varphi} x, e^{-i r \varphi} y, e^{i(r+s) \varphi} \zeta\right)
$$

which fixes only the singular point $p$. This action yields a natural action on the moduli space of vortices, and Euler character computations localize to the fixed point set. As shown at the end of section (5), the fixed point set in the moduli space of vortices is discrete and consists of vortex configurations centered at the singular point $p$. Since $p$ is away from the boundary of $\mathrm{D}$, the localization computation of the partition function (4.6) yields the same answer as the localization computation for vortices on the open curve $C$. Therefore for computational purposes one may work with stable pairs on $C$. This yields an explicit computational algorithm for the terms $f_{l, m}(q)$ in (4.6) which is summarized below.

The first term $f_{0,1}(q)$ in (4.6) represents the contribution of topologically trivial gauge field configurations. All terms $f_{0, m}(q), m \geq 2$ are obviously zero since the trivial line bundle has multiplicity 1 at $p$. Just as in the smooth case, $f_{0,1}(q)$ is given by the Hilbert series of the ring $R_{C}$ of regular functions on $C$. Since $(r, s)$ are coprime, the curve $C$ may be given in parametric form as $(x, y)=\left(t^{s}, t^{r}\right)$. Therefore $R_{C}$ is isomorphic to the subring $\mathbb{C}\left[t^{r}, t^{s}\right] \subset \mathbb{C}[t]$ spanned polynomials of the form $p\left(t^{r}, t^{r}\right)$ with $p(x, y)$ an arbitrary polynomial of two variables. It will be convenient 
to identify the set of monomials $t^{n} \in \mathbb{C}\left[t^{r}, t^{s}\right]$ with the set of exponents $n \in \mathbb{Z}_{\geq 0}$, which will be denoted by $\Lambda(r, s)$. Note that the complement $\Xi(r, s)=\mathbb{Z}_{\geq 0} \backslash \Lambda(r, s)$ is a finite set. Therefore $f_{0,1}(q)$ can be identified with the germ turn is generated by $x, y$ with weights $s, r$ respectively, modulo a relation of degree $s r$ :

$$
f_{0,1}(q)=\sum_{n \in \Lambda(r, s)} q^{n}=\frac{\left(1-q^{r s}\right)}{\left(1-q^{r}\right)\left(1-q^{s}\right)}=\frac{1}{1-q}-\sum_{n \in \Xi(r, s)} q^{n} .
$$

By comparison with the formula (4.5) it follows that the effect of the singularity in the topologically trivial sector is to remove the states in the Hilbert space of the harmonic oscillator with quantum numbers $n \in \Xi(p, q)$.

For concretness, suppose $(r, s)=(4,3)$. Then $\Lambda(4,3)$ is the set

$$
0, \quad 3,4, \quad 6,7, \cdots
$$

and the complement $\Xi(3,4)$ is the finite set

$$
1,2, \quad 5 .
$$

Therefore in this case

$$
f_{0,1}(q)=\frac{1}{1-q}-\left(q+q^{2}+q^{5}\right)=\frac{1-q+q^{3}-q^{5}+q^{6}}{1-q} .
$$

The terms $f_{l, m}(q)$ corresponding to topologically nontrivial sectors are constructed in a similar manner in terms of partial fillings of $\Lambda(r, s)$. A partial filling of $\Lambda(r, s)$ is a subset

$$
\Lambda(r, s) \subseteq \Lambda^{\prime}(r, s) \subseteq \mathbb{Z}_{\geq 0}
$$

with the property that if $\Lambda^{\prime}(r, s)$ contains some $n^{\prime} \in \Xi(r, s)$, then it must contain all its translates $n^{\prime}+n$ by arbitrary elements $n \in \Lambda(r, s)$. Each partial filling is obtained by adding finitely many elements in $\Xi(r, s)$ to $\Lambda(r, s)$ subject to this selection rule. For example all possible partial fillings in the case $(r, s)=(4,3)$ are

$$
\begin{array}{ll}
\Lambda^{\prime}(4,3)_{(1)}: & \mathbf{0 ,} \underline{\mathbf{1}}, \quad 3,4, \underline{\mathbf{5}}, 6,7, \ldots \\
\Lambda^{\prime}(4,3)_{(2)}: & \mathbf{0}, \quad \underline{\mathbf{2}}, 3,4, \underline{\mathbf{5}}, 6,7, \ldots \\
\Lambda^{\prime}(4,3)_{(3)}: & \mathbf{0}, \quad 3,4, \underline{\mathbf{5}}, 6,7, \ldots \\
\Lambda^{\prime}(4,3)_{(4)}: & \mathbf{0 ,} \underline{\mathbf{1}}, \underline{\mathbf{2}}, 3,4, \underline{\mathbf{5}}, 6,7, \ldots
\end{array}
$$

the extra elements being underlined in each case. What this means is the for example for $\Lambda^{\prime}(4,3)_{(1)}$ the line bundle has one additional section $s^{\prime}$ represented by $\underline{1}$, which does not vanish at the origin as we put $x=y=0$. The additional element in the ring given by $s^{\prime} y$ given by $\underline{\underline{5}}$, does vanish at the origin, as it vanishes as we set $y=0$. Similar considerations apply to the rest. A disallowed filling is for example

$$
0,1, \quad 3,4, \quad, 6,7, \ldots
$$

since the translation of 1 by 4 is $1+4=5$, which is missing in the above sequence. This is consistent with the fact that we can multiply a section by the holomorphic functions of $x, y$ and still get a section of the same bundle, and so 5 should also have been in the sequence of the sections of the line bundle.

Note that any partial filling $\Lambda^{\prime}(r, s)$ contains a unique finite subset $\Gamma(r, s)$ consisting of all elements $n^{\prime}$ which cannot be decomposed as

$$
n^{\prime}=n^{\prime \prime}+n
$$


D.-E. DIACONESCU, V. SHENDE, C. VAFA

with $n^{\prime \prime} \in \Lambda^{\prime}(r, s)$ and $n \in \Lambda(r, s), n \neq 0$. Moreover it is easy to show that any element $n^{\prime} \in \Lambda^{\prime}(r, s)$ can be written as $n^{\prime}=n^{\prime \prime}+n$ with $n^{\prime \prime} \in \Gamma(r, s)$ and $n \in \Lambda(r, s)$. The elements of $\Gamma(r, s)$ will be called the generators of $\Lambda^{\prime}(r, s)$. In the above example the generators are marked in each case with boldface characters.

The first Chern class $l$ of the vortex corresponding to $\Lambda^{\prime}(r, s)$ is the number of elements in the complement $\Lambda^{\prime}(r, s) \backslash \Lambda(r, s)$, which is the same as the number of additional sections we have introduced while the multiplicity $m$ is the number of generators, which is also the number of sections which do not vanish at $p$ as we set $x=y=0$. In the above example,

$$
l_{(1)}=l_{(2)}=2, \quad l_{(3)}=1, \quad l_{(4)}=3 .
$$

and

$$
m_{(1)}=m_{(2)}=m_{(3)}=2, \quad m_{(4)}=3 .
$$

Note that 0 is always a generator, and never an extra element. The pair $(l, m)$ assigned to a partial filling $\Lambda^{\prime}(r, s)$ will be called below the type of the partial filling.

The terms $f_{(l, m)}(q)$ are then obtained by summing the Hilbert series of all modules associated to partial fillings $\Lambda^{\prime}(r, s)$ of fixed type $(l, m)$. That is

$$
f_{(l, m)}(q)=q^{l} \sum_{\Lambda^{\prime}(r, s)} \sum_{n \in \Lambda^{\prime}(r, s)} q^{n}
$$

The factor $q^{l}$ reflects the fact that all such configurations have first Chern class $l$. For $(r, s)=(4,3)$ the resulting contributions are

$$
\begin{aligned}
& f_{1,2}(q)=q \sum_{n \in \Lambda^{\prime}(4,3)_{(3)}} q^{n}=q\left(\frac{1}{1-q}-q-q^{2}\right) \\
& f_{2,2}(q)=q^{2} \sum_{n \in \Lambda^{\prime}(4,3)_{(1)}} q^{n}+q^{2} \sum_{n \in \Lambda^{\prime}(4,3)_{(2)}} q^{n}=\left(\frac{1}{1-q}-q^{2}\right)+q^{2}\left(\frac{1}{1-q}-q\right) \\
& f_{3,3}(q)=q^{3} \sum_{n \in \Lambda^{\prime}(4,3)_{(4)}} q^{n}=\frac{q^{3}}{1-q}
\end{aligned}
$$

all other terms being trivial. Then the coefficients $f_{m}(q)$ in equation (4.7) are

$$
\begin{aligned}
& f_{1}(q)=\frac{1-q+q^{3}-q^{5}+q^{6}}{1-q} \\
& f_{2}(q)=\frac{q+q^{2}-q^{3}+q^{4}+q^{5}}{1-q} \\
& f_{3}(q)=\frac{q^{3}}{1-q}
\end{aligned}
$$

The HOMFLY polynomial of the $(r, s)$ torus knot is given by

$$
H_{(r, s)}(q, Q)=\left(\frac{Q}{q}\right)^{(r-1)(s-1) / 2} \frac{1}{1-q^{r}} \sum_{j=0}^{r-1} \frac{q^{s j+(r-1-j)(r-j) / 2}}{[j] ![r-1-j] !} \prod_{i=j+1-r}^{j}\left(q^{i}-Q\right) .
$$


where $[0] !=1$ and $[j] !=\left(1-q^{j}\right)[j-1]$ ! for all $j \geq 1$. Then a straightforward computation yields

$$
\sum_{m=1}^{3}(1-Q)^{m} f_{m}(q)=\left(\frac{q}{Q}\right)^{6} H_{(4,3)}(q, Q),
$$

confirming large $N$ duality for the $(4,3)$ torus knot. Note that [32, Thm. 19] proves the agreement between formula (4.10) and the stable pair localization computation for all $(r, s)$. The examples considered in this section are meant to explain the localization computation in a physical context.

The next case treated explicitly here is $(r, s)=(2,2 k+1), k \geq 1$. Then the HOMFLY polynomial (4.10) reduces to

$$
\begin{aligned}
H_{(2,2 k+1)}(q, Q) & =\left(\frac{Q}{q}\right)^{k} \frac{1-Q}{1-q} \frac{1-q^{2 k+2}-q Q\left(1-q^{2 k}\right)}{1-q^{2}} \\
& =\left(\frac{Q}{q}\right)^{k} \frac{1-Q}{1-q}\left[1+(q-Q) \sum_{j=0}^{k-1} q^{2 j}\right] .
\end{aligned}
$$

The subset $\Lambda(2 k+1,2) \subset \mathbb{Z}_{\geq 0}$ consists of the following elements

$$
0, \quad 2, \quad \cdots \quad 2 k, 2 k+1,2 k+2, \cdots
$$

its complement $\Xi(2 k+1,2)$ being

$$
1, \quad 3, \quad \cdots \quad 2 k-1 .
$$

There are $k+1$ partial fillings $\Lambda^{\prime}(2 k+1,2)_{(j)}, 0 \leq j \leq k+1$ as follows

$$
\begin{aligned}
\Lambda^{\prime}(2 k+1,2)_{(0)} & =\Lambda(2 k+1,2), \\
\Lambda^{\prime}(2 k+1,2)_{(1)} & =\Lambda(2 k+1,2) \cup\{1, \ldots, 2 k-1\}, \\
& \vdots \\
\Lambda^{\prime}(2 k+1,2)_{(j)} & =\Lambda(2 k+1,2) \cup\{2 j-1, \ldots, 2 k-1\}, \\
& \vdots \\
\Lambda^{\prime}(2 k+1,2)_{(k)} & =\Lambda(2 k+1,2) \cup\{2 k-1\} .
\end{aligned}
$$

Each $\Lambda^{\prime}(2 k+1,2)_{(j)}, 1 \leq j \leq k$ has two generators, $0,2 j-1$ and the complement of $\Lambda(2 k+1,2)$ contains $k-j+1$ elements. Therefore

$$
l_{(j)}=k-j+1, \quad m_{(j)}=2
$$

for all $1 \leq j \leq k$. Obviously, $l_{(0)}=0, m_{(0)}=1$. Therefore

$$
\begin{array}{r}
f_{1}(q)=\sum_{n \in \Lambda(2 k+1,2)} q^{n}=\frac{1}{1-q}-\sum_{j=1}^{k} q^{2 j-1}=\frac{1+q^{2 k+1}}{1-q^{2}} \\
f_{2}(q)=\sum_{j=1}^{k} q^{k-j+1}\left(\frac{1}{1-q}-\sum_{i=1}^{j-1} q^{2 i-1}\right) \\
=\sum_{j=1}^{k} q^{k-j+1} \frac{1+q^{2 j-1}}{1-q^{2}}=\frac{q\left(1-q^{2 k}\right)}{(1-q)\left(1-q^{2}\right)} .
\end{array}
$$


Note that

$$
f_{1}(q)+f_{2}(q)=\frac{1-q^{2 k+2}}{(1-q)\left(1-q^{2}\right)}
$$

Then a straightforward computation yields

$$
\sum_{m=1}^{2}(1-Q)^{m} f_{m}(q)=\left(\frac{q}{Q}\right)^{k} H_{(2,2 k+1)}(q, Q)
$$

\section{A SUMMARY FOR MATHEMATICIANS}

This section recapitulates the previous, in language perhaps more amenable to mathematicians. As before, the goal is to explain how a conjecture of Oblomkov and the second author 32 is related to a certain series of string dualities. On the one hand, this provides a physics proof of the conjecture. On the other, the conjecture was proven (in the mathematical sense) for torus knots in 32. This may then be viewed as confirmatory evidence for the string dualities which occur in the discussion below.

We recall the conjecture in question. Let $C$ be a curve in $\mathbb{C}^{2}$, say given by $f(x, y)=0$. Assume $C$ passes through the origin. Then the intersection of $C$ with the boundary of a small ball around the origin gives a link in the 3-sphere. Note this link has a natural orientation since it bounds a complex variety, and in fact a natural framing (though we will not use this). Recall that the HOMFLY polynomial is an invariant of links which assigns to a link $L$ a certain rational function $H(L)$ in the variables $q^{ \pm 1 / 2}, Q^{ \pm 1 / 2}$, characterized by the following skein relation:

$$
\begin{aligned}
Q^{1 / 2} H\left(\chi^{\searrow}\right)-Q^{-1 / 2} H(\nwarrow) & =\left(q^{1 / 2}-q^{-1 / 2}\right) H()() \\
Q^{1 / 2}-Q^{-1 / 2} & =\left(q^{1 / 2}-q^{-1 / 2}\right) H(\bigcirc)
\end{aligned}
$$

On $C$, we consider the moduli space $C^{[n]}$ parameterizing pairs $(F, s)$, where $F$ is a torsion free sheaf, $s$ is a section $s: \mathcal{O}_{C} \rightarrow F$, and $\operatorname{dim} F / s \mathcal{O}_{C}=n$. Note that in 32 the same notation was used for the Hilbert scheme of $n$ points on $C$; as shown in [36] these spaces are isomorphic for Gorenstein (and in particular planar) curves $C$. By $C_{0}^{[n]}$ we denote the space of such pairs in which the section vanishes only at the origin. By $C_{0}^{[n] ; m}$ we denote the locus where $m=\operatorname{dim}_{\mathbb{C}} F /(x, y) F$. Let $\mu=\operatorname{dim} \mathbb{C}[[x, y]] /\left(\partial_{x} f, \partial_{y} f\right)$ be the Milnor number of the singular point. We can now state:

Conjecture 5.1. 32.

$$
H(\text { the link of } C)=\left(\frac{Q}{q}\right)^{\mu-1} \sum_{n, m} q^{n}(1-Q)^{m} \chi\left(C_{0}^{[n] ; m}\right)
$$

One contribution of the present article is to explain how a certain sequence of string dualities connects the left of the conjecture to the right. The HOMFLY polynomial enters physics through Witten's observation 42. that it computes the expectation value of the knot viewed as a Wilson line in the Chern-Simons gauge theory on the three sphere. Here the gauge group is $U(N)$, and its holonomy around the knot is traced in the fundamental representation. Witten later [43] explained that this theory was equivalent to the type IIA topological string theory on $T^{*} S^{3}$, with $N$ lagrangian D-branes on $S^{3}$. The Wilson loop expectations (and hence the 
HOMFLY polynomial) are reproduced by introducing 34 the conormal bundle of the knot as a lagrangian brane $N^{*} K \subset T^{*} S^{3}$ and counting open strings with one end on $N^{*} K$ and the other on $S^{3}$.

As $N$ grows large one may take the view 13 that the $S^{3}$ shrinks and the space $X=T^{*} S^{3}$ is ultimately replaced by the small resolution of the conifold, i.e., by the total space $Y$ of the bundle $\mathcal{O}(-1) \oplus \mathcal{O}(-1)$ over $\mathbb{P}^{1}$. The D-branes on $S^{3}$ vanish along with the $S^{3}$. Attempting to follow $N^{*} K$ through the conifold transition is problematic, since it meets the collapsing $S^{3}$. Instead, it is better to first deform it off the $S^{3}$. In the case that the knot $K$ arose as an algebraic knot, we have explicitly constructed such a deformation in section 3.2 and followed it through the conifold transition. The essential feature of the resulting $L_{K} \subset Y$ is that it intersects the fiber over infinity in a single circle; the unique holomorphic curve passing through this circle is the singular curve itself. Conjecturally this curve is in fact the only irreducible curve with boundary on $Y$; this is proven in the next section in the case where $K$ arose from the curve $x^{r}=y^{s}$.

At this stage we see that the HOMFLY polynomial of $K$ should be computed by counting curves in $Y$ with boundary on $L_{K}$. The mathematical foundations of open Gromov-Witten theory are not presently available, but nonetheless for torus knots it is possible to describe the inevitable result of torus localization of the virtual class as in 18. This is done in the subsequent section, yielding agreement with known formulas for the HOMFLY polynomial in these cases.

According to [12, 34, 22, 29, we may lift to $M$-theory. Indeed, the topological string computes certain supersymmetric quantities in the full type IIA string theory on $Y \times \mathbb{R}^{3,1}$, which in turn is viewed as a limit of M-theory on $Y \times \mathbb{R}^{4,1}$. The variables work out so that the coefficient of $q^{r} Q^{s}$ in $(1-q) H$ counts certain M2-branes. More precisely, one considers an M5-brane $L \times \mathbb{R}^{2,1}$ for some $\mathbb{R}^{2,1} \subset \mathbb{R}^{4,1}$. Note that this brane breaks the symmetry group of the $\mathbb{R}^{4,1}$ to $\operatorname{Spin}(2,1) \times \operatorname{Spin}(2)$; we will only be interested in the $\operatorname{Spin}(2) \times \operatorname{Spin}(2)$ action. This group acts on all spaces of BPS states of M2 branes with boundary on this M5 brane, so these acquire a bigrading by the characters $q, t$ of the group. (Here $t$ is the character of the rotation transverse to the Lagrangian.) The M2 brane states also carry two additional gradings, corresponding to the class of the brane in $H_{2}(Y, L)=\mathbb{Z} \mathbb{P}^{1} \oplus \mathbb{Z D}$, where $\mathrm{D}$ is the class of the singular disc bounding the lagrangian. Writing $N_{d^{\prime}, d, \sigma, j}$ for the space of states of character $q^{\sigma} t^{j}$ and homology class $d \mathbb{P}^{1}+d^{\prime} \mathrm{D}$, the prediction of the above dualities is that the HOMFLY polynomial is (upto an appropriate $q^{\circ} Q^{*}$ ) given by

$$
H(q, Q)=\frac{1}{1-q} \sum N_{1, d, \sigma, j} Q^{d} q^{\sigma}(-1)^{2 j+1}
$$

The geometry of $M 2$-branes is not well enough understood that the $N_{1, d, \sigma, j}$ may be computed directly. However, according to [12, 34, 22, 29] the above index may be computed in a different type IIA limit of the M-theory, in which one of the dimensions of the $\mathbb{R}^{4}$ is compactified on a circle, and the different momenta modes around this circle are converted into bound states of D0-branes to D2-branes. The D2-branes must of course still have boundary along the Lagrangian. (The reason one is free to compute in any limit one likes is that all the states in question are BPS.) In the large volume limit, the space of D2-D0 branes is understood to be mathematically modelled by the space of stable pairs [19, 20, 35, and the index above is just its (appropriately weighted) Euler characteristic. These spaces are 
not identical to the spaces $C_{0}^{[n] ; m}$. In an upcoming paper of the first author, they will be shown to be related by wall crossing, and as a consequence conjecture 5.1 will be deduced from (5.3).

Here we argue instead at the level of M-theory. Since the coefficient of $Q^{d}$ is counting bound states formed by one M2-brane wrapping D and $d$ M2 branes wrapping $\mathbb{P}^{1}$, we must analyse how this configuration may occur. It is already known that M2 branes wrapping the $\mathbb{P}^{1}$ may not bind to each other; this for instance follows by running through the above series of dualities for the partition function of ChernSimons theory itself; the consequence being that in the absence of Lagrangians there is a unique BPS state consisting of a single M2 brane wrapping the $\mathbb{P}^{1}$. Thus each of the M2 branes wrapping the $\mathbb{P}^{1}$ must bind to $\mathrm{D}$.

Let $g_{m}(q)$ be the generating polynomial of which the coefficient of $q^{n}$ is the number of spin $n$ M2 branes wrapping $\mathrm{D}$ which can bind up to $m \mathrm{M} 2$ branes on $\mathbb{P}^{1}$. Then, since the M2 branes on $\mathbb{P}^{1}$ are indistinguishable and have fermion number -1 , the generating polynomial of bound states of such a brane to some number of branes wrapping $\mathbb{P}^{1}$ is just $(1-Q)^{m} g_{m}(q)$. Thus (5.3) can be rewritten as

$$
H(q, Q)=\frac{1}{1-q} \sum_{m} g_{m}(q)(1-Q)^{m}
$$

To relate this to conjecture [5.1] it remains only to explain why

$$
\frac{g_{m}(q)}{1-q}=\sum_{n} q^{n} \chi\left(C_{0}^{[n] ; m}\right)
$$

i.e., why the BPS M2-branes which can bind exactly $m$ M2 branes on $\mathbb{P}^{1}$ may be computed by stable pairs $s: \mathcal{O}_{C} \rightarrow F$ where $\operatorname{dim} F /(x, y) F=m$. As mentioned above, the relation to stable pairs and the appearance of the $1 /(1-q)$ is standard: the M2 brane has momentum modes around the circle which become D2-D0 bound states, and the particular index being computed becomes the Euler number of the stable pairs space. One might worry about the appropriate boundary conditions for the sheaf and the section. But whatever boundary conditions are chosen we will surely want all zeroes of the section $s$ to lie in the connected component $\mathrm{D}$ of $C \backslash L$ containing the origin. Assuming we choose $L$ sufficiently near the origin that $\mathrm{D}$ is contractible, then the space of such pairs contracts to $C_{0}^{[n]}$.

The essential thing to explain is what binding to $m$ branes on $\mathbb{P}^{1}$ has to do with the number of generators $\operatorname{dim} F /(x, y) F=m$. To count the number of ways an M2 brane on $\mathbb{P}^{1}$ may bind to a given M2 brane on $\mathrm{D}$, we first pass to the IIA theory and compute instead the number of ways the $\mathrm{D} 2$ brane on $\mathbb{P}^{1}$ may bind to a $\mathrm{D} 2$ brane $F$. The heuristic given in the previous section is that the virtual number of points on $F$ at the origin which are available for the branes on $\mathbb{P}^{1}$ is just $\operatorname{dim} F /(x, y) F$. To elaborate on this slightly, the "open strings are Ext" philosophy here specializes to the statement that the space of open strings from $F$ to a brane $\mathcal{O}_{\mathbb{P}^{1}}$ is $\operatorname{Ext}_{Y}^{1}\left(F, \mathcal{O}_{\mathbb{P}^{1}}\right)$. This immediately localizes to the intersection of $\mathbb{P}^{1}$ and $\mathrm{D}$; since this is a point the local to global spectral sequence collapses and we are reduced to computing Ext of modules in the complete local ring. Let us give coordinate $z$ to the $\mathbb{P}^{1}$ direction; then we are computing

$$
\operatorname{Ext}_{\mathbb{C}[[x, y, z]]}^{1}(F, \mathbb{C}[[z]])=\operatorname{Hom}_{\mathbb{C}[[x, y]]}(F, \mathbb{C})=(F /(x, y) F)^{\vee}
$$


One can also compute open strings in the other direction, $\operatorname{Ext}_{Y}^{1}\left(\mathcal{O}_{\mathbb{P}^{1}}, F\right)$; the result is that this canonically parameterizes the nontrivial syzygies of the completion of $F$ as a module over $\mathbb{C}[x, y]$. Because the curve is planar, this space has the same dimension as that parameterizing the generators.

We make a quick note about the sample computations in the previous section of the right hand side of conjecture 5.1. The main point is a certain invariant introduced there did not in fact require the existence of a torus action. Let $R$ be the complete local ring of the singularity; then the Jacobian factor parameterizes $R$-modules $M$ such that $\mathbb{C}[[t]] \supset M \supset R$. Note that given a stable pair $(M, s)$ one has an abstract isomorphism $M \otimes_{R} \mathbb{C}((t))=\mathbb{C}((t))$; requiring $s \mapsto 1$ fixes the isomorphism. In other words a stable pair with quotient supported at 0 is equivalent data to a rank one $R$-submodule of $\mathbb{C}((t))$. Let $M$ be such a module, then $M \mathbb{C}[[t]]=t^{-k} \mathbb{C}[[t]]$ for some $k$, and $\mathbb{C}[[t]] \subset t^{k} M \subset R$. Thus there is a map from the space of stable pairs to the Jacobian factor. The fiber over some module $M$ is just the set of elements in $M$, up to constant multiple. It is straightforward to see that the space of elements with leading term $t^{a}$ is a vector space and hence has Euler characteristic one. On the other hand $\operatorname{dim}_{\mathbb{C}} M / t^{a} R=a+\operatorname{dim}_{\mathbb{C}} M / R$. Thus the contribution of $M$ to the Euler numbers of pairs spaces is $q^{\operatorname{dim}_{\mathbb{C}} M / R} \mathcal{H}_{M}(q)$, where $\mathcal{H}_{M}(q)$ is the Hilbert function of $M$. Note that $\operatorname{dim}_{\mathbb{C}} M / R$ is the "l(M)" of the previous section. Let us also write $m(M)$ for the number of generators, and $\mathcal{J}^{l ; m}$ for the locus in the Jacobian factor of modules with $m$ generators. Then the $f_{m}$ of the previous section are

$$
f_{m}(q)=\sum_{n} q^{n} \chi\left(C_{0}^{[n] ; m}\right)=\int_{\mathcal{J} ; m} q^{\ell(M)} \mathcal{H}_{M}(q) d \chi(M)
$$

The integral is with respect to Euler characteristic, and has the meaning that we sum possible $\mathcal{H}_{M}(q)$ weighted by the Euler characteristic of the locus of modules with this Hilbert series. One may as desired further stratify by $l$, and introduce

$$
f_{l ; m}=\int_{\mathcal{J}^{l ; m}} \mathcal{H}_{M}(q) d \chi(M)
$$

in order to write

$$
\sum_{m, n} q^{n}(1-Q)^{m} \chi\left(C_{0}^{[n] ; m}\right)=\sum_{l, m} q^{l}(1-Q)^{m} f_{l, m}(q)
$$

\section{LARGE $N$ DUALITY AND TOPOLOGICAL AMPLITUdES FOR TORUS KNOTS}

The main goal of this section is to generalize the large $N$ duality results for the unknot reviewed in section (2) to arbitrary $(s, r)$ torus knots. The open topological A-model amplitudes for lagrangian cycles associated to torus knots will be explicitly computed on both sides of the transition employing an equivariant virtual localization approach analogous to [18. Note that the mirror topological B-model has been studied in [5], reproducing the HOMFLY polynomials via a matrix model approach.

The first task, carried out in section (6.1), is to write down an explicit analytic presentation the lagrangian cycles $L_{\mu} \subset X_{\mu}$, constructed in section (3) and show 
that they are preserved by the circle action

$$
(x, y, z, w) \mapsto\left(e^{i s \varphi} x, e^{i r \varphi} y, e^{-i s \varphi} z, e^{-i r \varphi} w\right) .
$$

Then it shown in section (6.2) that on the deformation side the open string instanton corrections to Chern-Simons theory are encoded in a formula of the form (2.19). The next section (6.3) contains the virtual localization computation of GromovWitten invariants on the resolution $Y$ with lagrangian boundary conditions on $M_{\epsilon}$. In particular it is shown that $M_{\epsilon}$ is preserved by the circle induced by (6.1) and the disc $D_{\epsilon}$ obtained in section (3.3) is the only circle invariant Riemann surface in $Y$ with boundary in $M_{\epsilon}$. The tangent-obstruction complex for circle invariant stable maps with lagrangian boundary conditions on $M_{\epsilon}$ is derived by linearizing the defining equations of $M_{\epsilon}$ near the one-cycle $\eta_{\epsilon}=\partial \mathrm{D}_{\epsilon}$. A by-product of this computation is an argument proving that the disc $D_{\epsilon}$ is rigid as a Riemann surface with boundary on $M_{\epsilon}$, even in the absence of the circle action. The final details of the localization computation are given in section (6.4). The main result is that the winding number one $\mathbf{A}$-model partition function of the lagrangian cycle $M_{\epsilon}$ is in agreement with the HOMFLY polynomial of the $(s, r)$ torus knot up to an overall sign depending on orientations. The proof is essentially an open A-model reflection of the Chern-Simons $S$-matrix formula [39, 5] relating the HOMFLY polynomial of a torus knot to the colored invariants of the unknot [39, [5].

6.1. Lagrangian cycles for torus knots. Lagrangian cycles for torus knots are obtained as a special case of the construction explained in section (3.2) for general algebraic knots. Consider the family of the curves $Z_{\mu} \subset X_{\mu}$

$$
Z_{\mu}: \quad x^{r}-\alpha^{r} y^{s}=0, \quad z^{r}-\alpha^{r}(-w)^{s}=0
$$

where $(r, s)$ are coprime integers with $r>s \geq 1$ or $r=s=1$, and $\alpha \in \mathbb{R} \backslash\{0\}$ is a fixed nonzero real number. As explained in section (3.2), the specialization of $Z_{\mu}$ at $\mu=0$ has $r-s+1$ connected components classified by the distinct roots of the equation $\eta^{r}-\alpha^{2 r}(-\eta)^{s}=0$. The component corresponding to $\eta=0$ is a union of two irreducible components $C^{ \pm}$given by

$$
x^{r}-\alpha^{r} y^{s}=0, \quad z=w=0,
$$

respectively

$$
z^{r}-\alpha^{r}(-y)^{s}=0, \quad x=y=0 .
$$

The remaining $r-s$ are disjoint smooth components isomorphic to $\mathbb{C}^{\times}$. Let $\gamma^{+}$: $S^{1} \rightarrow X$ be a parametric presentation of the intersection $\phi_{0}\left(C^{+} \backslash\{0\}\right) \cap P_{a}$, where $P_{a} \subset X$ is the sphere bundle $|\vec{v}|=a$. Its inverse image $\phi_{0}^{-1} \circ \gamma^{+}: S^{1} \rightarrow X_{0}$ has a parametric presentation of the form

$$
(x, y, z, w)=\left(\alpha b_{1}^{s} e^{i s \theta}, b_{1}^{r} e^{i r \theta}, 0,0\right)
$$

where $b_{1}$ must be a solution of the equation

$$
b_{1}^{2 r}+\alpha^{2} b_{1}^{2 s}=4 a .
$$

Some elementary real analysis shows that this equation has a unique positive real solution for any fixed $\alpha \neq 0, a>0$. Then the construction of section (3.1) then yields a lagrangian cycle $L_{\gamma^{+}} \subset X$. It will be checked below that $L_{\gamma^{+}}$does not intersect the zero section, hence its inverse image $L_{0}=\phi_{0}^{-1}\left(L_{\gamma^{+}}\right)$is a lagrangian cycle on $X_{0}$ supported away from the conifold singularity. 
For $\mu>0$ the connected components of $Z_{\mu}$ are in one-to-one correspondence with distinct roots of the equation $\eta^{r}-\alpha^{2 r}(\mu-\eta)^{s}=0$. Each such component is given by

$$
(x, y, z, w)=\left(t^{s}, t^{r}, \eta t^{-s},(\mu-\eta) t^{-r}\right) .
$$

In particular for sufficiently small $\mu>0$ there exists a continuous family $\eta(\mu)$ of roots specializing to $\eta=0$ at $\mu=0$. Let $C_{\mu} \subset X_{\mu}$ be the corresponding components of $Z_{\mu}$. Each connected component of the intersection $C_{\mu} \cap \phi_{\mu}^{-1}\left(P_{a}\right)$ must be an orbit of the circle action (6.1) since both $C_{\mu}, \phi_{\mu}^{-1}\left(P_{a}\right) \subset X_{\mu}$ are invariant cycles. Taking into account the parametric presentation of $C_{\mu}$, each intersection component must be of the form

$$
(x, y, z, w)=\left(\alpha b_{1}^{s} e^{i s \theta}, b_{1}^{r} e^{i r \theta}, \alpha b_{2}^{s} e^{-i s \theta},-b_{2}^{r} e^{-i r \theta}\right)
$$

with $\theta$ an angular coordinate on $S^{1}$ and $b_{1}, b_{2} \in \mathbb{R}_{>0}$. The parameters $b_{1}, b_{2}$ must satisfy the condition

$$
\alpha^{2}\left(b_{1}^{s}+b_{2}^{s}\right)^{2}+\left(b_{1}^{r}+b_{2}^{r}\right)^{2}=2\left(\mu+\sqrt{\mu^{2}+4 a^{2}}\right)
$$

which follows from the defining equation of $\phi_{\mu}^{-1}\left(P_{a}\right) \subset X_{\mu}$, and

$$
\alpha^{2}\left(b_{1} b_{2}\right)^{s}+\left(b_{1} b_{2}\right)^{r}=\mu,
$$

which follows from $x z-y w=\mu$. By continuity, for sufficiently small $\mu>0$ the intersection of $\phi_{\mu}\left(C_{\mu}\right)$ with $P_{a}$ consists of two connected one-cycles $\gamma_{\mu}^{ \pm}$conjugate under the antiholomorphic involution $\tau_{\mu}$ defined in (2.8). Moreover $\gamma_{\mu}^{+}$specializes to the cycle $\gamma^{+}$constructed above, while $\gamma_{\mu}^{-}$specializes to its conjugate, which is the intersection of $\phi_{0}\left(C^{-} \backslash\{0\}\right)$ with $P_{a}$. In fact this picture can be confirmed by detailed analytic computations which will be omitted in the interest of brevity.

Applying the construction of section (3.2) to the one-cycles $\gamma_{\mu}^{+}: S^{1} \rightarrow X$ yields a family of lagrangian cycles $L_{\gamma_{\mu}^{+}} \subset X$. The lagrangian cycles $L_{\mu} \subset X_{\mu}$ are the inverse images, $L_{\mu}=\phi_{\mu}^{-1}\left(L_{\gamma_{\mu}^{+}}\right)$, via the symplectomorphisms $\phi_{\mu}: X_{\mu} \rightarrow X$.

The next task is to check that the lagrangian cycles $L_{\mu}, L_{0}$ are invariant under the circle action (6.1) and do not intersect the zero section. Since the arguments are very similar, it suffices to present the details in one case only, say $L_{\mu}$. The explicit form of the circle action on $X$ is

$$
\begin{array}{ll}
{\left[\begin{array}{l}
u_{1} \\
u_{2}
\end{array}\right] \mapsto R(s \varphi)\left[\begin{array}{l}
u_{1} \\
u_{2}
\end{array}\right],} & {\left[\begin{array}{l}
u_{3} \\
u_{4}
\end{array}\right] \mapsto R(r \varphi)\left[\begin{array}{l}
u_{3} \\
u_{4}
\end{array}\right]} \\
{\left[\begin{array}{l}
v_{1} \\
v_{2}
\end{array}\right] \mapsto R(s \varphi)\left[\begin{array}{l}
v_{1} \\
v_{2}
\end{array}\right],} & {\left[\begin{array}{l}
v_{3} \\
v_{4}
\end{array}\right] \mapsto R(r \varphi)\left[\begin{array}{l}
v_{3} \\
v_{4}
\end{array}\right]}
\end{array}
$$

where

$$
R(\varphi)=\left[\begin{array}{cc}
\cos (\varphi) & -\sin (\varphi) \\
\sin (\varphi) & \cos (\varphi)
\end{array}\right]
$$

According to equations (3.1), the defining equation of $L_{\gamma_{\mu}^{+}}$is

$$
\vec{u}=\vec{f}(\theta), \quad \dot{\vec{f}}(\theta) \cdot(\vec{v}-\vec{g}(\theta))=0,
$$

where the functions $\vec{f}(\theta), \vec{g}(\theta)$ are determined by equation (6.5). One then finds (6.9)

$$
\left[\begin{array}{l}
f_{1}(\theta) \\
f_{2}(\theta)
\end{array}\right]=\alpha \frac{b_{1}^{s}+b_{2}^{s}}{c} R(s \theta)\left[\begin{array}{l}
1 \\
0
\end{array}\right] \quad\left[\begin{array}{l}
f_{3}(\theta) \\
f_{4}(\theta)
\end{array}\right]=-\frac{b_{1}^{r}+b_{2}^{r}}{c} R(r \theta)\left[\begin{array}{l}
1 \\
0
\end{array}\right]
$$


and

$$
\left[\begin{array}{l}
g_{1}(\theta) \\
g_{2}(\theta)
\end{array}\right]=-\alpha \frac{b_{1}^{s}-b_{2}^{s}}{4} c R(s \theta)\left[\begin{array}{l}
0 \\
1
\end{array}\right] \quad\left[\begin{array}{l}
g_{3}(\theta) \\
g_{4}(\theta)
\end{array}\right]=\frac{b_{1}^{r}-b_{2}^{r}}{4} c R(r \theta)\left[\begin{array}{l}
0 \\
1
\end{array}\right]
$$

with $c=\sqrt{2\left(\mu+\sqrt{\mu^{2}+4 a^{2}}\right)}$. Then it is straightforward to check that $L_{\gamma_{\mu}^{+}}$is preserved by the torus action using the elementary identity $R(\varphi) R\left(\varphi^{\prime}\right)=R\left(\varphi+\varphi^{\prime}\right)$. In fact $L_{\gamma_{\mu}^{+}}$admits a parameterization of the form

$$
\vec{u}=\vec{f}(\theta), \quad\left[\begin{array}{l}
v_{1} \\
v_{2}
\end{array}\right]=R(s \theta)\left[\begin{array}{l}
v_{10} \\
v_{20}
\end{array}\right] \quad\left[\begin{array}{l}
v_{3} \\
v_{4}
\end{array}\right]=R(r \theta)\left[\begin{array}{l}
v_{30} \\
v_{40}
\end{array}\right],
$$

where $\left(v_{10}, \ldots, v_{40}\right)$ are real parameters satisfying

$$
\begin{aligned}
\alpha\left(b_{1}^{s}+b_{2}^{s}\right) v_{10}-\left(b_{1}^{r}+b_{2}^{r}\right) v_{30} & =0 \\
s \alpha\left(b_{1}^{s}+b_{2}^{s}\right) v_{20}-r\left(b_{1}^{r}+b_{2}^{r}\right) v_{40} & =-\frac{c}{4}\left[\alpha s\left(b_{1}^{2 s}-b_{2}^{2 s}\right)+r\left(b_{1}^{2 r}-b_{2}^{2 r}\right)\right] .
\end{aligned}
$$

The first equation in (6.11) follows from the defining equation $\vec{u} \cdot \vec{v}=0$ of $X$, and the second from the equation $\dot{\vec{f}}(\theta) \cdot(\vec{v}-\vec{g}(\theta))=0$. Note that equations (6.11) define a real 2-plane in the fiber of $T^{*} S^{3}$ over the point $\vec{u}_{0}=\vec{f}(0)$. The points in this plane are in one-to-one correspondence with orbits of the circle action on the lagrangian cycle.

Note also that the intersection of $L_{\gamma_{\mu}^{+}}$with the zero section $\vec{v}=0$ is determined by the equation

$$
\dot{\vec{f}}(\theta) \cdot \vec{g}(\theta)=0
$$

which yields

$$
s \alpha^{2}\left(b_{1}^{2 s}-b_{2}^{2 s}\right)+r\left(b_{1}^{2 r}-b_{2}^{2 r}\right)=0 .
$$

Since $b_{1}, b_{2}$ satisfy simultaneously equations (6.6)-(6.7), equation (6.12) will have no solutions for generic values of $\mu, a>0$. Therefore in the generic case, this intersection with the zero section is empty.

6.2. Open string A-model on the deformation. Now consider an open Amodel with target space $X_{\mu}$ and lagrangian branes on the lagrangian cycles $L_{\mu}, S_{\mu}$, where $L_{\mu}$ is defined in equations (6.9), (6.10) and $S_{\mu} \simeq S^{3}$ is the fixed point set of the antiholomorphic involution (2.8) on $X_{\mu}$. Note that both cycles are preserved by the circle action (6.1). Moreover, $L_{\mu}$ intersects an irreducible component of the curve (6.2) along an orbit (6.5) of the $S^{1}$-action. Then it follows that the holomorphic cylinder $C_{\mu}$ given by

$$
(x, y, z, w)=\left(\alpha b_{1}^{s} t^{s}, b_{1}^{r} t^{r}, \alpha b_{2}^{s} t^{-s},-b_{2}^{r} t^{-r}\right),
$$

with

$$
\sqrt{\frac{b_{2}}{b_{1}}} \leq|t| \leq 1
$$

has boundary components on $S_{\mu}, L_{\mu}$ respectively. Obviously, $\mathrm{C}_{\mu}$ is preserved by the circle action (6.1). Set $\alpha=1$ in the following.

Equation (6.13) describes a circle invariant genus zero stable map to $X_{\mu}$ with two boundary components mapped to $S_{\mu}, L_{\mu}$. According to [43] such instantons are expected to generate Wilson loop corrections to the Chern-Simons action. If $C_{\mu}$ is the only torus invariant holomorphic cylinder in $X_{\mu}$ with boundary components 
on $L_{\mu}, S_{\mu}$, these corrections can be easily evaluated by a virtual localization computation analogous to [18. Such a computation has been carried out for example in [10] in a similar context. As required in section (2.1), the final formula for the instanton series is of the form

$$
Z_{\text {op-inst }}\left(g_{s}, \mathrm{q}\right)=\sum_{n \geq 1} \frac{e^{-t_{\mathrm{c}}}}{n} \operatorname{Tr}\left(U^{n}\right) \operatorname{Tr}\left(V^{n}\right)
$$

where $U, V$ are the holonomy of the gauge fields on $S_{\mu}, L_{\mu}$ about the boundary components of $C_{\mu}$, and $t_{C}$ is the symplectic area of $C_{\mu}$.

It remains to show that $C_{\mu}$ is indeed the unique torus invariant cylinder in $X_{\mu}$ with boundary components in $S_{\mu}, L_{\mu}$ respectively. The argument is analogous with [9], although more technically involved since the present torus action allows continuous families of invariant curves on $X_{\mu}$. The main steps will be summarized below omitting many computational details.

First note that any invariant map $\mathbb{C}^{\times} \rightarrow X_{\mu}$ must be of the form

$$
t \mapsto(x, y, z, w)=\left(\alpha_{1} t^{s}, \alpha_{2} t^{r}, \alpha_{3} t^{-s},-\alpha_{4} t^{-r}\right)
$$

where $\alpha_{1}, \ldots, \alpha_{4}$ are constant parameters satisfying

$$
\alpha_{1} \alpha_{3}+\alpha_{2} \alpha_{4}=\mu \text {. }
$$

Let $C_{\vec{\alpha}} \subset X_{\mu}, \vec{\alpha}=\left(\alpha_{1}, \ldots, \alpha_{4}\right)$ denote the image of this map. Since $L_{\mu}, S_{\mu}$ are preserved by the circle action, any connected of the intersection of $C_{\vec{\alpha}}$ with the lagrangian cycles must be an orbit of the form $t=\rho e^{i \theta}$, with $\rho \in \mathbb{R}_{>0}$. Then equations (6.9)-6.11) for for $L_{\mu}$ imply the following conditions

$$
\rho_{L}^{s} \alpha_{1}+\rho_{L}^{-s} \bar{\alpha}_{3}=\frac{A}{c}\left(b_{1}^{s}+b_{2}^{s}\right), \quad \rho_{L}^{r} \alpha_{2}+\rho_{L}^{-r} \bar{\alpha}_{4}=\frac{A}{c}\left(b_{1}^{r}+b_{2}^{r}\right) .
$$

where $\rho_{L}$ is the radius of a component of the intersection $C_{\vec{\alpha}} \cap L_{\mu}$ and $A=$ $\sqrt{\left(\left|\alpha_{1} \rho_{L}^{s}+\bar{\alpha}_{3} \rho_{L}^{-s}\right|^{2}+\left|\alpha_{2} \rho_{L}^{r}+\bar{\alpha}_{4} \rho_{L}^{-r}\right|^{2}\right) / 2}$. At the same time any connected component of the intersection $C_{\vec{\alpha}} \cap L_{\mu}$ must satisfy

$$
\alpha_{1} \rho_{S}^{2 s}=\bar{\alpha}_{3}, \quad \alpha_{2} \rho_{S}^{2 r}=\bar{\alpha}_{4},
$$

where $\rho_{S}$ denotes again the radius of the orbit. Equations 6.17), 6.18 imply that $\alpha_{i}, i=1, \ldots, 4$ must be non-zero real numbers, if $C_{\vec{\alpha}}$ intersects both $S_{\mu}, L_{\mu}$ nontrivially. For example, if $\alpha_{1}=0$, it follows easily that all the remaining coefficients $\alpha_{2}, \ldots, \alpha_{4}$ must be also trivial, which contradicts relation (6.16). Then by a reparametrization of the domain, the map (6.15) can be set in the form

$$
t \rightarrow\left(\alpha \beta_{1}^{s} t^{s}, \beta_{2}^{r} t^{r}, \alpha \beta_{2}^{s} t^{-s},-\beta_{2}^{r} t^{-r}\right)
$$

with $\beta_{1}, \beta_{2} \in \mathbb{R}_{>0}, \alpha \in \mathbb{R} \backslash\{0\}$, the intersections with $S_{\mu}, L_{\mu}$ being given by

$$
|t|=\sqrt{\frac{\beta_{2}}{\beta_{1}}}, \quad|t|=1
$$

respectively. Using again equations (6.9)-6.11) for for $L_{\mu}$, one finds the following nonempty intersection conditions

$$
\begin{gathered}
\frac{\alpha}{c^{\prime}}\left(\beta_{1}^{s}+\beta_{2}^{s}\right)=\frac{1}{c}\left(b_{1}^{s}+b_{2}^{s}\right), \quad \frac{1}{c^{\prime}}\left(\beta_{1}^{r}+\beta_{2}^{r}\right)=\frac{1}{c}\left(b_{1}^{r}+b_{2}^{r}\right) . \\
-\frac{\alpha c^{\prime}}{4}\left(\beta_{1}^{s}-\beta_{2}^{s}\right)=v_{20}, \quad \frac{c^{\prime}}{4}\left(\beta_{1}^{r}-\beta_{2}^{r}\right)=v_{40} .
\end{gathered}
$$




$$
\begin{gathered}
s\left(b_{1}^{s}+b_{2}^{s}\right) v_{20}-r\left(b_{1}^{r}+b_{2}^{r}\right) v_{40}=-\frac{c}{4}\left[s\left(b_{1}^{2 s}-b_{2}^{2 s}\right)+r\left(b_{1}^{2 r}-b_{2}^{2 r}\right)\right] \\
v_{10}=v_{30}=0, \quad v_{20}^{2}+v_{40}^{2}=a^{\prime 2} .
\end{gathered}
$$

where $\vec{v}_{0}$ is a vector in the plane (6.11) parametrizing a common circle orbit of $L_{\mu}$ and $C_{\vec{\alpha}}$, and

$$
a^{\prime}=\sqrt{a^{2}+\left|\vec{v}_{0}\right|^{2}}, \quad c^{\prime}=\sqrt{2\left(\mu+\sqrt{\mu^{2}+4 a^{\prime 2}}\right)} .
$$

Recall that the coefficients $b_{1}, b_{2}$ are given functions of $(\mu, a)$ determined by equations (6.6)-(6.7), as explained in section (6.1).

Next note that it suffices to show that the orbit parametrized by the vector $\vec{v}_{0}$ coincides with the boundary of the built in cylinder $\mathrm{C}_{\mu}$, since then the two cylinders must coincide by holomorphy. This follows by an elementary but fairly tedious computation in real analysis. The strategy is to solve for $\left(v_{20}, v_{40}\right)$ in equations (6.22) and substitute the solutions in equations (6.20)- (6.21). Then one solves for $\left(\alpha \beta_{1}^{s}, \alpha \beta_{2}^{s}\right)$ respectively $\left(\beta_{1}^{r}, \beta_{2}^{r}\right)$ in the resulting equations imposing at the same time the positivity conditions $\beta_{1}, \beta_{2}>0$. Note that this will yield a priori independent expressions of the form

$$
\beta_{i}^{r}=F_{i}\left(c^{\prime}, b_{1}, b_{2}\right), \quad \alpha \beta_{i}^{s}=G_{i}\left(c^{\prime}, b_{1}, b_{2}\right),
$$

$i=1,2$, where $F_{i}\left(c^{\prime}, a, \mu\right), G_{i}\left(c^{\prime}, a, \mu\right), i=1,2$ are explicit functions of $\left(c^{\prime}, a, \mu\right)$. Moreover the expressions (6.23) must satisfy the obvious compatibility condition

$$
F_{1}\left(c^{\prime}, b_{1}, b_{2}\right)^{s} G_{2}\left(c^{\prime}, b_{1}, b_{2}\right)^{r}=F_{2}\left(c^{\prime}, b_{1}, b_{2}\right)^{s} G_{1}\left(c^{\prime}, b_{1}, b_{2}\right)^{r} .
$$

A straightforward but fairly long computation shows that the matching condition (6.24) is equivalent to

$$
F_{+}(\eta)^{s} G_{+}(\eta)^{r}=F_{-}(\eta)^{s} G_{-}(\eta)^{r}
$$

where $\eta=c^{\prime 2}$,

$$
\begin{aligned}
& F_{ \pm}(\eta)=\left(1+\frac{r^{2} D^{2}}{s^{2}}\right) \frac{D \eta}{2 c} \mp \frac{r}{s} B D \pm\left[\left(1+\frac{r^{2} D^{2}}{s^{2}}\right)\left(1+D^{2}\right)\left(\frac{\eta^{2}}{4 c^{2}}-\widetilde{\mu} \eta\right)-B^{2}\right]^{1 / 2} \\
& G_{ \pm}(\eta)=\left(1+\frac{r^{2} D^{2}}{s^{2}}\right) \frac{\eta}{2 c} \pm B \pm \frac{r D}{s}\left[\left(1+\frac{r^{2} D^{2}}{s^{2}}\right)\left(1+D^{2}\right)\left(\frac{\eta^{2}}{4 c^{2}}-\widetilde{\mu} \eta\right)-B^{2}\right]^{1 / 2}
\end{aligned}
$$

and

$$
D=\frac{b_{1}^{r}+b_{2}^{r}}{b_{1}^{s}+b_{2}^{s}} \quad B=\frac{c}{2}\left(\frac{b_{1}^{s}-b_{2}^{s}}{b_{1}^{s}+b_{2}^{s}}+\frac{r}{s} D \frac{b_{1}^{r}-b_{2}^{r}}{b_{1}^{s}+b_{2}^{s}}\right)
$$

On then has to analyze the monotonicity properties of the functions $F_{ \pm}(\eta), G_{ \pm}(\eta)$ on the intervals where $\beta_{1}, \beta_{2}>0$. Suppressing the details, which are quite elementary, it follows that for sufficiently small $\mu>0$ equation (6.24) admits only the solution $c^{\prime}=c$, if $a>0$ is in addition bounded above by a constant $a_{0}(r, s)$ depending only on $r, s$. Returning to the expressions (6.23), this implies in turn that $\beta_{i}=b_{i}$ for $i=1,2$. Therefore the two orbits indeed coincide. 
6.3. Open Gromov-Witten invariants on the resolution. The goal of this section is to compute the Gromov-Witten invariants for stable maps $f: \Sigma \rightarrow Y$ with lagrangian boundary conditions on the cycle $M_{\epsilon}$ constructed in section (3.3) for a polynomial $f(x, y)$ of the form

$$
f(x, y)=x^{r}-y^{s},
$$

with $r>s \geq 1$ coprime. These invariants will be computed assuming the existence of a virtual fundamental cycle and a virtual localization result for the moduli space of such maps, by analogy with [18.

Recall that the main steps in the construction of $M_{\epsilon} \subset Y$ are as follows. Let $C^{+} \subset X_{0}$ be the plane curve determined by

$$
f(x, y)=0, \quad z=w=0
$$

in the singular conifold $X_{0}$. Let $\gamma^{+}: S^{1} \rightarrow X=T^{*} S^{3}$ be the one-cycle obtained by intersecting the sphere bundle $P_{a}, a>0$ with the image $\phi_{0}\left(C^{+}\right)$, where $\phi_{0}$ : $X_{0} \rightarrow X$ is the symplectomorphism constructed below equation (2.6). Let $\gamma_{\epsilon}^{+}=$ $\phi_{0} \circ \varrho_{\epsilon} \circ \phi_{0}^{-1} \circ \gamma^{+}$be the the dilation of $\gamma^{+}$via the radial map $\varrho_{\epsilon}: X_{0} \backslash\{0\} \rightarrow X_{0}(\epsilon)$,

$$
\varrho_{\epsilon}(x, y, z, w)=\left(x, \frac{\sqrt{|z|^{2}+|y|^{2}+\epsilon^{2}}}{\sqrt{|z|^{2}+|y|^{2}}} y, \frac{\sqrt{|z|^{2}+|y|^{2}+\epsilon^{2}}}{\sqrt{|z|^{2}+|y|^{2}}} z, w\right) .
$$

Applying the construction in section (3.1) to $\gamma_{\epsilon}^{+}$yields a lagrangian cycle $L_{\epsilon} \subset X$. As shown in equation (3.4), $M_{\epsilon}$ is the inverse image $\sigma^{-1} \circ \varrho_{\epsilon}^{-1} \circ \phi_{0}^{-1}\left(L_{\epsilon}\right)$.

The cycle $L_{\epsilon} \subset X$ admits an explicit parametric presentation analogous to the presentation of the cycles $L_{\mu} \subset X_{\mu}$ in section (6.1). Note that the one-cycle $\phi_{0}^{-1}\left(\gamma_{\epsilon}^{+}\right)=\phi_{0}^{-1}\left(\varrho_{\epsilon} \circ \gamma^{+}\right)$is parametrically given by

$$
(x, y, z, w)=\left(b_{1}^{s} e^{i s \theta}, \sqrt{b_{1}^{2 r}+\epsilon^{2}} e^{i r \theta}, 0,0\right),
$$

where $b_{1}=b_{1}^{+}(a)$ is the unique positive real solution of the equation

$$
b_{1}^{2 s}+b_{1}^{2 r}=4 a .
$$

Then $L_{\epsilon} \subset X$ is given by equations of the form

$$
\vec{u}=\vec{f}(\theta), \quad \dot{\vec{f}}(\theta) \cdot(\vec{v}-\vec{g}(\theta))=0
$$

where

$$
\begin{array}{cl}
{\left[\begin{array}{l}
f_{1}(\theta) \\
f_{2}(\theta)
\end{array}\right]=\frac{b_{1}^{q}}{c} R(s \theta)\left[\begin{array}{l}
1 \\
0
\end{array}\right]} & {\left[\begin{array}{l}
f_{3}(\theta) \\
f_{4}(\theta)
\end{array}\right]=-\frac{\sqrt{b_{1}^{2 r}+\epsilon^{2}}}{c} R(r \theta)\left[\begin{array}{l}
1 \\
0
\end{array}\right]} \\
{\left[\begin{array}{l}
g_{1}(\theta) \\
g_{2}(\theta)
\end{array}\right]=\frac{b_{1}^{s}}{4} c R(s \theta)\left[\begin{array}{l}
0 \\
1
\end{array}\right]} & {\left[\begin{array}{l}
g_{3}(\theta) \\
g_{4}(\theta)
\end{array}\right]=-\frac{\sqrt{b_{1}^{2 r}+\epsilon^{2}}}{4} c R(p \theta)\left[\begin{array}{l}
0 \\
1
\end{array}\right]}
\end{array}
$$

and $c=\sqrt{4 a+\epsilon^{2}}$. Using the above formulas, it is straightforward to show that $L_{\epsilon}$ is invariant under the circle action (6.8).

Now recall that the defining equations of $Y$ in $\mathbb{C}^{4} \times \mathbb{P}^{1}$ are

$$
x \lambda=w \rho, \quad y \lambda=z \rho
$$

where $[\lambda, \rho]$ are homogeneous coordinates on $\mathbb{P}^{1}$. There are two affine coordinate patches on $Y, U$ given by $\rho \neq 0$ with coordinates

$$
x, y, \zeta=\frac{\lambda}{\rho},
$$


and $U^{\prime} \subset y$ given by $\lambda \neq 0$, with coordinates

$$
z, w, \zeta^{\prime}=\frac{\rho}{\lambda} .
$$

Obviously, the transition functions are

$$
w=x \zeta, \quad z=y \zeta, \quad \zeta^{\prime}=\frac{1}{\zeta} .
$$

The strict transform $C \subset Y$ of $C^{+}$is contained in the first patch and has defining equations

$$
f(x, y)=0, \quad \zeta=0 .
$$

Moreover, note that equation (6.1) also defines a circle action on singular threefold $X_{0}$ which preserves $C^{+}$. This lifts to a circle action $S^{1} \times Y \rightarrow Y$,

$$
(x, y, z, w) \times[\lambda, \rho] \mapsto\left(e^{i s \varphi} x, e^{i r \varphi} y, e^{-i q \alpha} z, e^{-i p \alpha} w\right) \times\left[e^{-i(p+q) \alpha} \lambda, \rho\right],
$$

which preserves $C$. Since the blow-up map $\sigma: Y \rightarrow X_{0}$ and the dilation map (6.25) are equivariant, it follows that the action (6.28) preserves $M_{\epsilon}$. Therefore it also preserves the singular holomorphic disk $\mathrm{D}_{\epsilon}$ with boundary on $M_{\epsilon}$ obtained by intersecting $M_{\epsilon}$ and $C$. Note that $\mathrm{D}_{\epsilon}$ is given in parametric form by

$$
(x, y, \zeta)=\left(t^{s}, t^{r}, 0\right), \quad|t| \leq b_{1} .
$$

Next one has to show that (6.29) is the unique torus invariant disk instanton $f: \Delta \rightarrow Y$ with lagrangian boundary conditions on $M_{\epsilon}$. Using equations (6.27) it is straightforward to check that the only coordinate hyperplane in $Y$ intersecting $M_{\epsilon}$ nontrivially is $\lambda=0$, in which case the intersection is the one-cycle $\eta_{\epsilon}=\mathrm{D}_{\epsilon}$. All other coordinate hyperplanes, $x=0, y=0, \rho=0$ do not intersect $M_{\epsilon}$. In particular this implies the image $f(\Delta)$ of such a map cannot be contained in the surface $\rho=0$. Then torus invariance implies that $f(\Delta)$ is either disjoint from the surface $\rho=0$, or intersects it transversely at the torus fixed point $z=w=0, \rho=0$. In the first case the fixed point $t=0$ in the domain must be mapped to the fixed point $x=y=0, \lambda=0$ in the target. Moreover, in both cases, the restriction of the map $f$ to the punctured disk $\Delta \backslash\{0\}$ must be of the form

$$
(x, y, \zeta)=\left(\alpha_{1} t^{ \pm s}, \alpha_{2} t^{ \pm r}, \alpha_{3} t^{\mp(r+s)}\right)
$$

for some complex parameters $\left(\alpha_{1}, \alpha_{2}, \alpha_{3}\right)$.

If the first case holds, the map $f$ must be of the form

$$
(x, y, \zeta)=\left(\alpha_{1} t^{s}, \alpha_{2} t^{r}, 0\right)
$$

or

$$
(x, y, \zeta)=\left(0,0, \alpha_{3} t^{(r+s)}\right)
$$

since $f(0)=(0,0,0)$. The second subcase is ruled out because $M_{\epsilon}$ does not intersect the zero section $x=y=0$. In the first subcase the image $f(\Delta)$ is contained in the surface $\lambda=0$ which intersects $M_{\epsilon}$ along the boundary of $\mathrm{D}_{\epsilon}$. Therefore $f(\Delta)$ and $\mathrm{D}_{\epsilon}$ must have common boundary, which implies they must coincide.

The second case can hold only if $\alpha_{3} \neq 0$, which implies that the image $f(\Delta)$ cannot be contained in the surface $\lambda=0$. Then torus invariance implies that $f(\Delta)$ must be disjoint from the surface $\lambda=0$ since any common point would have to be a fixed point of the torus action. At the same time the only fixed point in the domain 
is mapped to the fixed point $z=w=0, \rho=0$. Therefore $f(\Delta)$ is contained in the coordinate chart $U^{\prime}$. In terms of the coordinates $\left(z, w, \zeta^{\prime}\right)$, equation (6.30) reads

$$
\left(z, w, \zeta^{\prime}\right)=\left(\alpha_{1} \alpha_{3} t^{\mp r}, \alpha_{2} \alpha_{3} t^{\mp s}, \alpha_{3}^{-1} t^{ \pm(r+s)}\right) .
$$

Since $\alpha_{3} \neq 0$, the condition $f(0)=(0,0,0)$ implies that $\alpha_{1}=\alpha_{2}=0$. This is again ruled out since $M_{\epsilon}$ does not intersect the zero section.

In conclusion, $\mathrm{D}_{\epsilon}$ is indeed the unique torus invariant holomorphic disc on $Y$ with boundary in $M_{\epsilon}$. Then the computation of Gromov-Witten invariants reduces to the computation of multicover contributions of $\mathrm{D}_{\epsilon}$ via a virtual localization theorem. One then requires an explicit form of lagrangian boundary conditions for an $S^{1}$-invariant stable map $f: \Sigma \rightarrow Y$ which factors through the disc $\mathrm{D}_{\epsilon} \subset Y$. Let Ann $\left.\left(M_{\epsilon}\right) \subset T^{*} Y\right|_{M_{\epsilon}}$ be the subbundle of the cotangent bundle of $Y$ which annihilates the tangent bundle $\left.T M_{\epsilon} \subset T Y\right|_{M_{\epsilon}}$. The boundary conditions are determined by a framing of $\left.\operatorname{Ann}\left(M_{\epsilon}\right)\right|_{\eta_{\epsilon}}$, that is three sections of $\left.T^{*} Y\right|_{\eta_{\epsilon}}$ which form a basis of $\operatorname{Ann}\left(M_{\epsilon}\right)$ at any point on $\eta_{\epsilon}=\partial \mathrm{D}_{\epsilon}$. This computation reduces basically to the linearization of the defining equations of $M_{\epsilon}$ in $Y$, which is standard differential geometry. Omitting the intermediate steps, the resulting generators are, in local coordinates $(x, y, \zeta)$,

$$
\begin{aligned}
\alpha=b_{1}^{s}[2 A C+ & \left.(s-r)\left(b_{1}^{2 r}+\epsilon^{2}\right) B+(s-r)^{2} b_{1}^{2 s}\left(b_{1}^{2 r}+\epsilon^{2}\right)\right]\left(e^{-i s \theta} d x+e^{i s \theta} d \bar{x}\right) \\
+b_{1}^{2 r}[B C & \left.-(s-r) b_{1}^{2 s} A+(s-r)^{2} b_{1}^{2 s}\left(b_{1}^{2 r}+\epsilon^{2}\right)\right]\left(e^{-i r \theta} d y+e^{i r \theta} d \bar{y}\right) . \\
\beta= & e^{-i(r+s) \theta} d \bar{\zeta}+\frac{b_{1}^{s} \sqrt{b_{1}^{2 r}+\epsilon^{2}}}{C}\left[\frac{B}{2 c^{2} b_{1}^{s}} e^{-i s \theta} d x+\frac{(s-r) b_{1}^{s}}{2 c^{2}} e^{i s \theta} d \bar{x}\right. \\
& -\frac{1}{4 c^{2} b_{1}^{r}}\left(\frac{2 b_{1}^{2 r}+\epsilon^{2}}{b_{1}^{2 r}+\epsilon^{2}} A+(s-r) \epsilon^{2}\right) e^{-i r \theta} d y \\
& \left.+\frac{1}{4 c^{2} b_{1}^{r}}\left(\frac{\epsilon^{2} A}{b_{1}^{2 r}+\epsilon^{2}}+(s-r)\left(2 b_{1}^{2 r}+\epsilon^{2}\right)\right) e^{i r \theta} d \bar{y}\right]
\end{aligned}
$$

where

$$
\begin{gathered}
A=2 s b_{1}^{2 s}+(r+s)\left(b_{1}^{2 r}+\epsilon^{2}\right), \quad B=(r+s) b_{1}^{2 s}+2 r\left(b_{1}^{2 r}+\epsilon^{2}\right) . \\
C=s b_{1}^{2 s}+r\left(b_{1}^{2 r}+\epsilon^{2}\right) .
\end{gathered}
$$

In particular, $\alpha$ is real and $\beta$ is complex.

6.3.1. Deformation theory. Let $\Delta \subset \mathbb{C}$ be the disk $|t| \leq b_{1}$. Let $f: \Delta \rightarrow Y$ be the map

$$
t \mapsto(x, y, \zeta)=\left(t^{s}, t^{r}, 0\right) .
$$

Obviously $f$ factors through the disk $C \subset Y$ mapping the boundary of the disk, $|t|=b_{1}$ to the boundary $\eta_{\epsilon}=\partial \subset \subset M_{\epsilon}$. Let $f_{\partial}$ denote the restriction of $f$ to the boundary. Let $\mathcal{T}_{(\Delta, f)}$ denote the sheaf of germs of holomorphic sections of the bundle $f^{*} T_{Y}$ satisfying the boundary conditions

$$
f_{\partial}^{*}(\alpha)\left(\left.s\right|_{\partial \Delta}\right)=0, \quad f_{\partial}^{*}(\beta)\left(\left.s\right|_{\partial \Delta}\right)=0,
$$

where $\alpha, \beta$ are the generators of the annihilator sub-bundle $\left.A n n\left(M_{\epsilon}\right)\right|_{\eta_{\epsilon}}$ given in equations (6.32)-6.33). Let $\mathcal{T}_{\Delta}$ be the sheaf of germs of holomorphic sections of the tangent bundle $T_{\Delta}$ satisfying the boundary condition

$$
\left.\gamma\right|_{\partial \Delta}\left(\left.s\right|_{\partial \Delta}\right)=0
$$


where $\gamma=t d \bar{t}+\bar{t} d t$.

The deformation complex of the stable map $(\Delta, f)$ with lagrangian boundary conditions along $M_{\epsilon}$ is

$$
\begin{aligned}
0 & \rightarrow H^{0}\left(\Delta, \mathcal{T}_{\Delta}\right) \rightarrow H^{0}\left(\Delta, \mathcal{T}_{(\Delta, f)}\right) \rightarrow \operatorname{Def}(\Delta, f) \\
& \rightarrow H^{1}\left(\Delta, \mathcal{T}_{\Delta}\right) \rightarrow H^{1}\left(\Delta, \mathcal{T}_{(\Delta, f)}\right) \rightarrow \operatorname{Obs}(\Delta, f) \rightarrow 0 .
\end{aligned}
$$

In particular one has to compute the Cech cohomology groups $H^{k}\left(\Delta, \mathcal{T}_{(\Delta, f)}\right)$ with $k=0,1$. This will be done below using the following open cover of $\Delta$

$$
U=\left\{t|0<| t \mid \leq b_{1}\right\}, \quad U^{\prime}=\left\{t|0 \leq| t \mid<b_{1}\right\} .
$$

Local sections over $U, U^{\prime}$ are of the form

$$
\begin{aligned}
& s=\sum_{n \in \mathbb{Z}}\left(a_{n} t^{n} \partial_{x}+b_{n} t^{n} \partial y+c_{n} t^{n} \partial_{\zeta}\right) \\
& s^{\prime}=\sum_{n \geq 0}\left(a_{n}^{\prime} t^{n} \partial_{x}+b_{n}^{\prime} t^{n} \partial y+c_{n}^{\prime} t^{n} \partial_{\zeta}\right)
\end{aligned}
$$

The coefficients $\left(a_{n}, n_{n}, c_{n}\right), n \in \mathbb{Z}$, are subject to boundary conditions of the form

$$
\begin{aligned}
\bar{c}_{-n} & =A_{1} a_{n-r}+A_{2} \bar{a}_{r+2 s-n}+B_{1} b_{n-s}+B_{2} \bar{b}_{2 r+s-n} \\
a_{s+n}+\bar{a}_{s-n} & =C_{1}\left(b_{r+n}+\bar{b}_{r-n}\right) .
\end{aligned}
$$

Changing the variable $n$ to $n+r+s$ in the first equation yields the equivalent formulation

$$
\begin{aligned}
\bar{c}_{-(n+r+s)} & =A_{1} a_{s+n}+A_{2} \bar{a}_{s-n}+B_{1} b_{r+n}+B_{2} \bar{b}_{r-n} \\
a_{s+n}+\bar{a}_{s-n} & =C_{1}\left(b_{r+n}+\bar{b}_{r-n}\right) .
\end{aligned}
$$

These conditions are derived from (6.35), the coefficients $A_{1}, A_{2}, B_{1}, B_{2}, C_{1}$ being determined from the explicit expressions of $\alpha^{\prime}, \beta^{\prime}$. The resulting functions of $\left(p, q, b_{1}, \epsilon\right)$ are fairly complicated, but explicit formulas for these coefficients will not be needed in the following. It suffices to note that the following conditions are satisfied

$$
\begin{gathered}
A_{1}^{2}-A_{2}^{2} \neq 0, \quad A_{1} C_{1}+B_{1} \neq 0, \quad A_{2} C_{2}+B_{2} \neq 0 \\
A_{1} C_{1}+B_{1}+A_{2} C_{2}+B_{2} \neq 0 .
\end{gathered}
$$

for sufficiently generic values of $\epsilon>0$. This will be assumed from now on.

The cohomology group $H^{0}\left(\Delta, \mathcal{T}_{(\Delta, f)}\right)$ is isomorphic to the kernel of the Cech differential, which consists of sections

$$
s=\sum_{n \geq \mathbb{Z}}\left(a_{n} t^{n} \partial_{x}+b_{n} t^{n} \partial y+c_{n} t^{n} \partial_{\zeta}\right)
$$

where $\left(a_{n}, b_{n}, c_{n}\right)$ are subject to the boundary conditions (6.36), and

$$
a_{n}=0, \quad b_{n}=0, \quad c_{n}=0
$$

for all $n<0$. The behavior of these equations depends on the value of $n$, resulting in several different cases. Recall that under the current assumption $r>s \geq 1$.

a) $n>r$. The boundary conditions (6.38) reduce to

$$
A_{1} a_{n+s}+B_{1} b_{n+r}=0, \quad a_{n+s}=C_{1} b_{n+r} .
$$

If $B_{1} \neq A_{1} C_{1}$, these equations admit only the trivial solution, hence $a_{n+s}=b_{n+r}=$ 0 for all $n>r$. 
b) $s<n \leq r$. The boundary conditions (6.38) reduce to

$$
A_{1} a_{n+s}+B_{1} b_{n+r}+B_{2} \bar{b}_{r-n}=0, \quad a_{n+s}=C_{1}\left(b_{n+r}+\bar{b}_{r-n}\right),
$$

which are equivalent to

$$
\left(A_{1} C_{1}+B_{1}\right) b_{n+r}+\left(A_{1} C_{1}+B_{2}\right) \bar{b}_{r-n}=0, \quad a_{n+s}=C_{1}\left(b_{n+r}+\bar{b}_{r-n}\right) .
$$

c) $-r \leq n<-s$. The boundary conditions become

$$
A_{2} \bar{a}_{s-n}+B_{1} b_{n+r}+B_{2} \bar{b}_{r-n}=0, \quad \bar{a}_{s-n}=C_{1}\left(b_{n+r}+\bar{b}_{r-n}\right),
$$

which are equivalent to

$$
\left(A_{2} C_{1}+B_{1}\right) b_{n+r}+\left(A_{2} C_{1}+B_{2}\right) \bar{b}_{r-n}=0, \quad \bar{a}_{s-n}=C_{1}\left(b_{n+r}+\bar{b}_{r-n}\right) .
$$

Now note that the first equations in (6.41) yield

$$
\left(A_{2} C_{1}+B_{1}\right) \bar{b}_{r-n}+\left(A_{2} C_{1}+B_{2}\right) b_{n+r}=0
$$

for all $s<n \leq r$, by complex conjugation and changing $n$ into $-n$. Therefore for any $s<n \leq r$ the following equations must hold simultaneously

$$
\begin{aligned}
& \left(A_{1} C_{1}+B_{1}\right) b_{n+r}+\left(A_{1} C_{1}+B_{2}\right) \bar{b}_{r-n}=0 \\
& \left(A_{2} C_{1}+B_{2}\right) b_{n+r}+\left(A_{2} C_{1}+B_{1}\right) \bar{b}_{r-n}=0
\end{aligned}
$$

This implies $b_{r+n}=b_{r-n}=0$ in this range, provided that

$$
\left(B_{1}-B_{2}\right)\left(\left(A_{1}+A_{2}\right) C_{1}+B_{1}+B_{2}\right) \neq 0,
$$

which is the case for generic $\epsilon$. The remaining equations in (6.40), (6.41) then imply that $a_{n+s}=0$ as well if $s<n \leq q$.

Therefore it has been proven so far that

$$
a_{n}=0, \quad \text { for } \quad n>2 s
$$

and

$$
b_{n}=0, \quad \text { for } n>r+s \quad \text { or } \quad 0 \leq n<r-s .
$$

$d)-(r+s)<n<-r$. Then the boundary condition yield

$$
A_{2} \bar{a}_{s-n}+B_{2} \bar{b}_{r-n}=0, \quad \bar{a}_{s-n}=C_{1} \bar{b}_{r-n} .
$$

As long as $A_{2} C_{1}+B_{2} \neq 0$, it follows that $b_{r-n}=0, a_{s-n}=0$ in this range. These results duplicate those obtained in case $(a)$.

$e)-s \leq n \leq s$. In this case the resulting equations are

$$
\begin{aligned}
A_{1} a_{n+s}+A_{2} \bar{a}_{q-n}+B_{1} b_{n+r}+B_{2} \bar{b}_{r-n} & =0 \\
a_{n+s}+\bar{a}_{s-n} & =C_{1}\left(b_{n+r}+\bar{b}_{r-n}\right) .
\end{aligned}
$$

Note that for any $-s \leq n \leq s$ the second equation in (6.43) is invariant under complex conjugation, followed by the reflection $n \rightarrow(-n)$. This is expected since it originates in the boundary condition given by the real one-form $\alpha^{\prime}$. For sufficiently generic coefficients, the first equation does not have this property. In fact, using this transformation, the first set of equations in (6.43) is equivalent to

$$
\begin{aligned}
A_{1} a_{n+s}+A_{2} \bar{a}_{s-n}+B_{1} b_{n+r}+B_{2} \bar{b}_{r-n} & =0, & & 0<n \leq s \\
A_{2} a_{n+s}+A_{1} \bar{a}_{s-n}+B_{2} b_{n+r}+B_{1} \bar{b}_{r-n} & =0, & & 0<n \leq s \\
A_{1} a_{s}+A_{2} \bar{a}_{s}+B_{1} b_{r}+B_{2} \bar{b}_{r} & =0, & &
\end{aligned}
$$


Assuming again $\epsilon$ to be sufficiently generic such that $A_{1}^{2} \neq A_{2}^{2}$, these equations determine $a_{n}, 0 \leq n \leq 2 s$ uniquely in terms of the variables $b_{n},(r-s) \leq n \leq r+s$ as follows:

$$
\begin{array}{ll}
a_{n+s}=\frac{\left(A_{2} B_{2}-A_{1} B_{1}\right) b_{n+r}+\left(A_{2} B_{1}-A_{1} B_{2}\right) \bar{b}_{r-n}}{A_{1}^{2}-A_{2}^{2}}, & 0 \leq n \leq q \\
\bar{a}_{s-n}=\frac{\left(A_{2} B_{1}-A_{1} B_{2}\right) b_{n+r}+\left(A_{2} B_{2}-A_{1} B_{1}\right) \bar{b}_{r-n}}{A_{1}^{2}-A_{2}^{2}}, & 0<n \leq s .
\end{array}
$$

Substituting in the second set of equations in (6.43) yields

$$
\left(\left(A_{1}+A_{2}\right) C_{1}+B_{1}+B_{2}\right)\left(b_{n+r}+\bar{b}_{r-n}\right)=0
$$

for $-s \leq n \leq s$. Since

$$
C_{1}\left(A_{1}+A_{2}\right)+B_{1}+B_{2} \neq 0
$$

generically, this implies the reality condition

$$
b_{n+r}+\bar{b}_{r-n}=0 .
$$

Therefore the space of solutions can be parameterized by the independent variables $b_{n}$ with $r-s \leq n \leq r$, where $b_{n} \in \mathbb{C}$ for $n \neq r$ and $b_{r} \in i \mathbb{R}$. The last case is

f) $n \leq-(r+s)$. Using the previous cases, the boundary conditions reduce to $c_{n}=0$ for all $n \geq 0$ and no additional conditions on $a_{n}, b_{n}$.

In conclusion the cohomology group $H^{0}\left(\Delta, \mathcal{T}_{(\Delta, f)}\right)$ is isomorphic to the space of sections of the form

$$
s=\sum_{n=0}^{2 s} a_{n} t^{n} \partial_{x}+\sum_{n=r-s}^{r+s} b_{n} t^{n} \partial_{y}
$$

where the coefficients $a_{n}, b_{n}$ are subject to conditions (6.44), (6.45).

Note that the above computation implies that the holomorphic cylinder D admits no finite deformations in $Y$ as a Riemann surface with boundary on $M_{\epsilon}$. The argument relies on the fact that the coefficients $c_{n}$ are zero for all infinitesimal deformations of the map $f: \Delta \rightarrow Y$. This implies that the disk $\mathrm{D}$ cannot be deformed in the normal directions to the plane $\zeta=0$. Any such deformation would yield by linearization an infinitesimal deformation with some $c_{n} \neq 0$. Therefore the disk $\mathrm{D}$ may admit only deformations in the plane $\zeta=0$. However note that the lagrangian cycle $M_{\epsilon}$ intersects the plane $\zeta=0$ along the boundary $\eta_{\epsilon}$ of $\mathrm{D}$. Hence any deformation of $\mathrm{D}$ would have to intersect $M_{\epsilon}$ along the same cycle $\eta_{\epsilon}$. Then the claim follows noting that any two irreducible holomorphic curves passing through the same circle must coincide.

The cohomology group $H^{1}\left(\Delta, \mathcal{T}_{(\Delta, f)}\right)$ is isomorphic to the cokernel of the Cech differential. The image of the differential map consists of sections of the form

$$
s=\sum_{n \in \mathbb{Z}}\left(a_{n} t^{n} \partial_{x}+b_{n} t^{n} \partial_{y}+c_{n} t^{n} \partial_{\zeta}\right)-\sum_{n \geq 0}\left(a_{n}^{\prime} t^{n} \partial_{x}+b_{n}^{\prime} t^{n} \partial_{y}+c_{n}^{\prime} t^{n} \partial_{\zeta}\right)
$$

on $U \cap U^{\prime}=\left\{t|0<| t \mid<b_{1}\right\}$, where the coefficients $\left(a_{n}, b_{n}, c_{n}\right), n \in \mathbb{Z}$, are subject to the conditions (6.38). The coefficients $\left(a_{n}^{\prime}, b_{n}^{\prime}, c_{n}^{\prime}\right), n \in \mathbb{Z}_{\geq 0}$ are arbitrary. In order to determine the cokernel, consider the equation

$$
s=\sum_{n \in \mathbb{Z}}\left(\alpha_{n} t^{n} \partial_{x}+\beta_{n} t^{n} \partial_{y}+\gamma_{n} t^{n} \partial_{\zeta}\right)
$$


in the variables $\left(a_{n}, b_{n}, c_{n}\right), n \in \mathbb{Z},\left(a_{n}^{\prime}, b_{n}^{\prime}, c_{n}^{\prime}\right), n \in \mathbb{Z}_{\geq 0}$, where $\left(\alpha_{n}, \beta_{n}, \gamma_{n}\right), n \in \mathbb{Z}$ are arbitrary coefficients. This implies

$$
\left(a_{n}, b_{n}, c_{n}\right)=\left(\alpha_{n}, \beta_{n}, \gamma_{n}\right)
$$

for all $n<0$ and

$$
\left(a_{n}-a_{n}^{\prime}, b_{n}-b_{n}^{\prime}, c_{n}-c_{n}^{\prime}\right)=\left(\alpha_{n}, \beta_{n}, \gamma_{n}\right)
$$

for all $n \geq 0$. The effect of boundary conditions must be analyzed again on a case by case basis, depending on the value of $n$.

$\left.a^{\prime}\right) r<n<r+s$. The boundary equations become

$$
\begin{aligned}
A_{1} a_{n+s}+A_{2} \bar{\alpha}_{s-n}+B_{1} b_{n+r}+B_{2} \bar{\beta}_{r-n} & =\bar{\gamma}_{-(n+r+s)} \\
a_{n+s}+\bar{\alpha}_{s-n} & =C_{1}\left(b_{n+r}+\bar{\beta}_{r-n}\right),
\end{aligned}
$$

which are equivalent to

$$
\begin{aligned}
\left(A_{1} C_{1}+B_{1}\right) b_{n+r}+\left(A_{2}-A_{1}\right) \bar{\alpha}_{s-n}+\left(A_{1} C_{1}+B_{2}\right) \bar{\beta}_{r-n} & =\bar{\gamma}_{-(n+r+s)} \\
a_{n+q}+\bar{\alpha}_{s-n}-C_{1}\left(b_{n+r}+\bar{\beta}_{r-n}\right) & =0 .
\end{aligned}
$$

$\left.b^{\prime}\right)-(r+s)<n<-r$. In this case the boundary conditions read

$$
\begin{aligned}
A_{1} \alpha_{n+s}+A_{2} \bar{a}_{s-n}+B_{1} \beta_{n+r}+B_{2} \bar{b}_{r-n} & =\bar{\gamma}_{-(n+r+s)} \\
\alpha_{n+s}+\bar{a}_{s-n} & =C_{1}\left(\beta_{n+r}+\bar{b}_{r-n}\right),
\end{aligned}
$$

and are equivalent to

$$
\begin{aligned}
\left(A_{2} C_{1}+B_{2}\right) \bar{b}_{r-n}+\left(A_{1}-A_{2}\right) \alpha_{n+s}+\left(A_{2} C_{1}+B_{1}\right) \beta_{n+r} & =\bar{\gamma}_{-(n+r+s)} \\
\bar{a}_{s-n}+\alpha_{n+s}-C_{1}\left(\beta_{n+r}+\bar{b}_{r-n}\right) & =0
\end{aligned}
$$

By complex conjugation and reflection, $n \rightarrow(-n)$, equations (6.49) yield

$$
\begin{aligned}
\left(A_{2} C_{1}+B_{2}\right) b_{n+r}+\left(A_{1}-A_{2}\right) \bar{\alpha}_{s-n}+\left(A_{2} C_{1}+B_{1}\right) \bar{\beta}_{r-n} & =\gamma_{n-(r+s)} \\
a_{n+s}+\bar{\alpha}_{s-n}-C_{1}\left(\bar{\beta}_{r-n}+b_{n+r}\right) & =0
\end{aligned}
$$

for all $r<n<r+s$. If

$$
A_{1} C_{1}+B_{1} \neq 0, \quad A_{2} C_{1}+B_{2} \neq 0,
$$

equations (6.49), (6.50) admit solutions if and only if the linear relation

$$
\begin{aligned}
& \left(A_{1} C_{1}+B_{1}\right)\left(A_{2} C_{1}+B_{2}\right)\left(\gamma_{n-(r+s)}-\bar{\gamma}_{-(n+r+s)}\right)= \\
& \left(B_{1}+B_{2}+C_{1}\left(A_{1}+A_{2}\right)\right)\left(\left(A_{1}-A_{2}\right) \bar{\alpha}_{s-n}+\left(B_{1}-B_{2}\right) \bar{\beta}_{r-n}\right) .
\end{aligned}
$$

holds.

$\left.c^{\prime}\right) n \geq r+s$. The boundary conditions are identical to case $\left(a^{\prime}\right)$ above.

$\left.d^{\prime}\right) n \leq-(r+s)$. The boundary conditions are very similar to case $\left(b^{\prime}\right)$, except the first equation in (6.49) now reads

$$
\left(A_{2} C_{1}+B_{2}\right) \bar{b}_{r-n}+\left(A_{1}-A_{2}\right) \alpha_{n+s}+\left(A_{2} C_{1}+B_{1}\right) \beta_{n+r}=\bar{c}_{-(n+r+s)} .
$$

By complex conjugation and reflection, $n \rightarrow(-n)$, this becomes

$$
\left(A_{2} C_{1}+B_{2}\right) b_{n+r}+\left(A_{1}-A_{2}\right) \bar{\alpha}_{s-n}+\left(A_{2} C_{1}+B_{1}\right) \bar{\beta}_{r-n}=c_{n-(r+s)} .
$$


This equation is very similar to the first equation in (6.50), except the right hand side is $c_{n-(r+s)}$ instead of $\gamma_{n-(r+s)}$. As a result the resulting system of linear equations in $b_{n+r}, c_{n-(r+s)}$,

$$
\begin{aligned}
\left(A_{1} C_{1}+B_{1}\right) b_{n+r}+\left(A_{2}-A_{1}\right) \bar{\alpha}_{s-n}+\left(A_{1} C_{1}+B_{2}\right) \bar{\beta}_{r-n} & =\bar{\gamma}_{-(n+r+s)} \\
\left(A_{2} C_{1}+B_{2}\right) b_{n+r}+\left(A_{1}-A_{2}\right) \bar{\alpha}_{s-n}+\left(A_{2} C_{1}+B_{1}\right) \bar{\beta}_{r-n}-c_{n-(r+s)} & =0
\end{aligned}
$$

admits solutions for any values of $\alpha_{s-n}, \beta_{r-n}$.

$\left.e^{\prime}\right) s<n \leq r$. In this case the boundary equations read

$$
\begin{aligned}
A_{1} a_{n+s}+A_{2} \bar{\alpha}_{s-n}+B_{1} b_{n+r}+B_{2} \bar{b}_{r-n} & =\bar{\gamma}_{-(n+r+s)} \\
a_{n+s}+\bar{\alpha}_{s-n} & =C_{1}\left(b_{n+r}+\bar{b}_{r-n}\right)
\end{aligned}
$$

and are equivalent to

$$
\begin{aligned}
\left(A_{1} C_{1}+B_{1}\right) b_{n+r}+\left(A_{1} C_{1}+B_{2}\right) \bar{b}_{r-n}+\left(A_{2}-A_{1}\right) \bar{\alpha}_{n-s} & =\bar{\gamma}_{-(n+r+s)} \\
a_{n+s}-C_{1}\left(b_{n+r}+\bar{b}_{r-n}\right)+\bar{\alpha}_{s-n} & =0 .
\end{aligned}
$$

$\left.f^{\prime}\right)-r \leq n<-s$.

$$
\begin{aligned}
A_{1} \alpha_{n+s}+A_{2} \bar{a}_{s-n}+B_{1} b_{n+r}+B_{2} \bar{b}_{r-n} & =\bar{\gamma}_{-(n+r+s)} \\
\alpha_{n+s}+\bar{a}_{s-n} & =C_{1}\left(b_{n+r}+\bar{b}_{r-n}\right)
\end{aligned}
$$

Again, by complex conjugation and reflection these equations become

$$
\begin{aligned}
A_{1} \bar{\alpha}_{s-n}+A_{2} a_{n+s}+B_{1} \bar{b}_{r-n}+B_{2} b_{n+r} & =\gamma_{n-(r+s)} \\
\bar{\alpha}_{s-n}+a_{n+s} & =C_{1}\left(\bar{b}_{r-n}+b_{n+r}\right)
\end{aligned}
$$

with $s<n \leq r$. They are equivalent to

$$
\begin{aligned}
\left(A_{2} C_{1}+B_{2}\right) b_{n+r}+\left(A_{2} C_{1}+B_{1}\right) \bar{b}_{r-n}+\left(A_{1}-A_{2}\right) \bar{\alpha}_{s-n} & =\gamma_{n-(r+s)} \\
a_{n+s}-C_{1}\left(\bar{b}_{r-n}+b_{n+r}\right)+\bar{\alpha}_{s-n} & =0 .
\end{aligned}
$$

Since $a_{n+s}, b_{n+r}, b_{r-n}$ are independent variables, equations 6.52), 6.53) admit solutions for any values of $\alpha_{r-n}, \gamma_{-(n+r+s)}, \gamma_{n-(r+s)}$. The remaining case is

$\left.g^{\prime}\right)-s \leq n \leq s$. In this range the boundary conditions read

$$
\begin{aligned}
A_{1} a_{n+s}+A_{2} \bar{a}_{s-n}+B_{1} b_{n+r}+B_{2} \bar{b}_{r-n} & =\bar{\gamma}_{-(n+r+s)} \\
a_{n+s}+\bar{a}_{s-n}-C_{1}\left(b_{n+r}+\bar{b}_{r-n}\right) & =0 .
\end{aligned}
$$

By complex conjugation and reflection, the first set of these equations is equivalent to

$$
\begin{array}{rlr}
A_{1} a_{n+s}+A_{2} \bar{a}_{s-n}+B_{1} b_{n+r}+B_{2} \bar{b}_{r-n} & =\bar{\gamma}_{-(n+r+s)}, \quad 0<n \leq s \\
A_{2} a_{n+s}+A_{1} \bar{a}_{s-n}+B_{2} b_{n+r}+B_{1} \bar{b}_{r-n} & =\gamma_{n-(r+s)}, \quad 0<n \leq s \\
A_{1} a_{s}+A_{2} \bar{a}_{s}+B_{1} b_{r}+B_{2} \bar{b}_{r}=\bar{\gamma}_{-(r+s)}, &
\end{array}
$$


If $A_{1}^{2} \neq A_{2}^{2}$, these equations yield

$$
\begin{array}{rlr}
a_{n+s}= & \frac{\left(A_{2} B_{2}-A_{1} B_{1}\right) b_{n+r}+\left(A_{2} B_{1}-A_{1} B_{2}\right) \bar{b}_{r-n}}{A_{1}^{2}-A_{2}^{2}}, & 0 \leq n \leq \\
& +\frac{A_{1} \bar{\gamma}_{-(n+r+s)}-A_{2} \gamma_{n-(r+s)}}{A_{1}^{2}-A_{2}^{2}} & \\
\bar{a}_{s-n}= & \frac{\left(A_{2} B_{1}-A_{1} B_{2}\right) b_{n+r}+\left(A_{2} B_{2}-A_{1} B_{1}\right) \bar{b}_{r-n}}{A_{1}^{2}-A_{2}^{2}}, & \\
& +\frac{A_{1} \gamma_{n-(r+s)}-A_{2} \bar{\gamma}_{-(n+r+s)}}{A_{1}^{2}-A_{2}^{2}}
\end{array}
$$

Substituting in the second set of equations in (6.54) yields

$$
\left(\left(A_{1}+A_{2}\right) C_{1}+B_{1}+B_{2}\right)\left(b_{n+r}+\bar{b}_{r-n}\right)=\gamma_{n-(r+s)}+\bar{\gamma}_{-(n+r+s)} .
$$

for $-s \leq n \leq s$. Since $b_{n+r}, b_{r-n}$ are independent variables, these equations admits solutions for any values of $\gamma_{n-(r+s)}, \bar{\gamma}_{-(n+r+s)}$ provided that $\left(A_{1}+A_{2}\right) C_{1}+B_{1}+$ $B_{2} \neq 0$.

Summarizing the above results, it follows that for sufficiently generic $\epsilon$ equation (6.47) admits solutions if and only if the coefficients $\left(\alpha_{n}, \beta_{n}, \gamma_{n}\right), n \in \mathbb{Z}$, satisfy the linear relations (6.51). This implies that the cohomology group $H^{1}\left(\Delta, \mathcal{T}_{(\Delta, f)}\right)$ is an $(s-1)$-dimensional complex vector space which can be identified with the space of sections of the form

$$
s=\sum_{n=1-s}^{-1} \gamma_{n} t^{n} \partial_{\zeta}
$$

on $U \cap U^{\prime}$. In particular, if $s=1$, this space is trivial.

For completeness, note that the computation of the cohomology groups $H^{i}\left(\Delta, \mathcal{T}_{\Delta}\right)$, $i=0,1$ is entirely analogous, and technically much simpler. One finds that $H^{0}\left(\Delta, \mathcal{T}_{\Delta}\right)$ is generated by sections of the form

$$
a_{-1} \partial_{t}+a_{0} t \partial_{t}+a_{1} t^{2} \partial_{t}
$$

with

$$
a_{-1}+\bar{a}_{1}=0, \quad a_{0}+\bar{a}_{0}=0 .
$$

The obstruction space $H^{1}\left(\Delta, \mathcal{T}_{\Delta}\right)$ is trivial.

6.3.2. Virtual localization. Now let $\overline{\mathcal{M}}_{g, 1}\left(Y, M_{\epsilon} ; d, 1\right)$ be the moduli space of genus $g \geq 0$ stable maps with $h=1$ boundary components mapped to $M_{\epsilon}$, in the relative homology class $d\left[C_{0}\right]+[\mathrm{C}] \in H_{2}\left(Y, M_{\epsilon}\right), d \in \mathbb{Z}_{\geq 0}$. The circle action

$$
(x, y, \zeta) \mapsto\left(e^{-i s \varphi} x, e^{-i r \varphi} y, e^{i(r+s) \varphi} \zeta\right)
$$

on $Y$ preserves $M_{\epsilon}$, hence it induces an action on the moduli space of stable maps. Let also $\bar{M}_{g, 1}(Y, d)$ denote the moduli space of genus $g$ stable maps to $Y$ with one marked point in the homology class $d\left[C_{0}\right] \in H_{2}(Y)$. This moduli space is equipped with a natural evaluation map at the marked point, ev $: \bar{M}_{g, 1}(Y, d) \rightarrow Y$.

A map $f: \Sigma \rightarrow Y$ determines a circle fixed point in the moduli space $\overline{\mathcal{M}}_{g, 1}\left(Y, M_{\epsilon} ; d, 1\right)$ if and only if there exists a circle action on the domain $\Sigma$ such that $f$ is equivariant. This implies that domain must be a union $\Sigma=\Sigma_{0} \cup_{\nu} \Delta$ where $\Sigma_{0}$ is a closed nodal Riemann surface without boundary which intersects the disk $\Delta$ at a single point $\nu$, which is a simple node of $\Sigma$. Moreover the image of the 
restriction $\left.f\right|_{\Delta}$ must coincide with the holomorphic disc $\mathrm{D}$, which has been shown below (6.29) to be the unique torus invariant disc in $Y$ with boundary in $M_{\epsilon}$. In more detail, the following conditions must hold

- $\Delta$ admits a parameterization $\Delta=\left\{|t| \leq r_{1}\right\}$ such that $\nu$ is identified with the point $t=0$ and

$$
\left.f\right|_{\Delta}(t)=\left(t^{s}, t^{r}, 0\right)
$$

The circle action on $\Delta$ is given by $t \mapsto e^{-i \varphi} t$.

- Note that there is an algebraic torus actions $\mathbb{C}^{\times} \times Y \rightarrow Y$ which agrees with above real torus action by restriction to the unit circle. Then the data $\left(\Sigma_{0}, f_{0}, \nu\right)$, with $f_{0}=\left.f\right|_{\Sigma}$ must be a $\mathbb{C}^{\times}$-invariant stable map to $Y$ such that $f_{0}(\nu)=p$, where $p \in Y$ is the point $x=y=\zeta=0$.

These conditions imply that the fixed locus $\overline{\mathcal{M}}_{g, 1}\left(Y, M_{\epsilon} ; d, 1\right)^{S^{1}}$ is isomorphic to the fixed subspace

$$
e v_{\nu}^{-1}(p)^{\mathbb{C}^{\times}} \subset \bar{M}_{g, 1}(Y, d)^{\mathbb{C}^{\times}}
$$

The deformation complex of a fixed stable map $(\Sigma, f)$ is

$$
\begin{aligned}
0 & \rightarrow \operatorname{Aut}(\Sigma) \rightarrow \operatorname{Def}(f) \rightarrow \operatorname{Def}(\Sigma, f) \\
& \rightarrow \operatorname{Def}(\Sigma) \rightarrow \operatorname{Obs}(f) \rightarrow \operatorname{Obs}(\Sigma, f) \rightarrow 0 .
\end{aligned}
$$

where the notation is self-explanatory. All terms carry natural circle actions since $(\Sigma, f)$ is a circle invariant map. The fixed part of the deformation complex determines the virtual fundamental cycle on the fixed locus, while the moving part determines the virtual normal bundle to the fixed locus. Each term will be analyzed below assuming that $\Sigma_{0}$ is nonempty. In the special case $\Sigma_{0}=\emptyset$ the deformation complex (6.57) reduces to (6.37) analyzed in the previous subsection.

Given the structure of fixed maps explained above, there is an exact sequence

$$
\begin{aligned}
0 & \rightarrow \operatorname{Def}(f) \rightarrow H^{0}\left(\Delta, \mathcal{T}_{\left(\Delta,\left.f\right|_{\Delta}\right)}\right) \oplus \operatorname{Def}\left(f_{0}\right) \rightarrow T_{p} Y \\
& \rightarrow \operatorname{Obs}(f) \rightarrow H^{1}\left(\Delta, \mathcal{T}_{\left(\Delta,\left.f\right|_{\Delta}\right)}\right) \oplus \operatorname{Obs}\left(f_{0}\right) \rightarrow 0 .
\end{aligned}
$$

This yields the following relations in the representation ring of the circle

$$
\begin{aligned}
\operatorname{Obs}(f)^{f}-\operatorname{Def}(f)^{f}= & H^{1}\left(\Delta, \mathcal{T}_{\left(\Delta,\left.f\right|_{\Delta}\right)}\right)^{f}-H^{0}\left(\Delta, \mathcal{T}_{(\Delta, f \mid \Delta)}\right)^{f} \\
& +\operatorname{Obs}\left(f_{0}\right)^{f}-\operatorname{Def}\left(f_{0}\right)^{f} \\
\operatorname{Obs}(f)^{m}-\operatorname{Def}(f)^{m}= & H^{1}\left(\Delta, \mathcal{T}_{\left(\Delta,\left.f\right|_{\Delta}\right)}\right)^{m}-H^{0}\left(\Delta, \mathcal{T}_{\left(\Delta,\left.f\right|_{\Delta}\right)}\right)^{m} \\
& +\operatorname{Obs}\left(f_{0}\right)^{m}-\operatorname{Def}\left(f_{0}\right)^{m}+T_{p} Y .
\end{aligned}
$$

Moreover standard arguments imply

$$
\begin{aligned}
\operatorname{Aut}(\Sigma)^{f, m} & =\operatorname{Aut}\left(\Sigma_{0}, \nu\right)^{f, m}+\operatorname{Aut}(\Delta, 0)^{f, m} \\
\operatorname{Def}(\Sigma)^{f} & =\operatorname{Def}\left(\Sigma_{0}, \nu\right)^{f} \\
\operatorname{Def}(\Sigma)^{m} & =\operatorname{Def}\left(\Sigma_{0}, \nu\right)^{m}+T_{\nu} \Sigma_{0} \otimes T_{0} \Delta
\end{aligned}
$$

while the cohomology groups $H^{0}\left(\Delta, \mathcal{T}_{\left(\Delta,\left.f\right|_{\Delta}\right)}\right)$ have been determined in equations (6.46), (6.56). There is however a discrete ambiguity in reading off their equivariant content, reflecting a choice of orientation on the moduli space of stable maps with lagrangian boundary conditions [18. As explained in [18, the difference between these choices is encoded in an overall sign which cannot be fixed in the absence of a rigorous construction of the moduli space equipped with a virtual cycle. Therefore the present computation will be a test of large $N$ duality up to sign. Given equations 
(6.44), 6.45), the deformation space (6.46) is isomorphic to a vector space of the form

$$
\mathbb{R}\left\langle\partial_{y}\right\rangle \oplus \bigoplus_{n=r-s}^{r-1} \mathbb{C}\left\langle t^{n} \partial_{y}\right\rangle .
$$

At the same time, the obstruction space (6.56) is naturally identified with the complex vector space

$$
\bigoplus_{n=1-s}^{-1} \mathbb{C}\left\langle t^{n} \partial_{\zeta}\right\rangle .
$$

This yields the following relations in the representation ring of $S^{1}$

$$
\begin{aligned}
H^{0}\left(\Delta, \mathcal{T}_{(\Delta, f \mid \Delta)}\right)^{m}=\sum_{n=1}^{s} R^{n}, & H^{1}\left(\Delta, \mathcal{T}_{(\Delta, f \mid \Delta)}\right)^{m} & =\sum_{n=1}^{s-1} R^{-(r+n)}, \\
H^{0}\left(\Delta, \mathcal{T}_{(\Delta, f \mid \Delta)}\right)^{f}=\mathbb{R}, & H^{1}\left(\Delta, \mathcal{T}_{(\Delta, f \mid \Delta)}\right)^{f} & =0,
\end{aligned}
$$

where $R$ is the canonical representation of $S^{1}$ on $\mathbb{C}$, and $\mathbb{R}$ denotes the trivial real representation. Note also that $\operatorname{Aut}(\Delta)$ is isomorphic to the space of sections of $\mathcal{T}_{\Delta}$ of the form $a \partial_{t}+b t \partial_{t}$ with $a \in \mathbb{C}, b \in i \mathbb{R}$. Therefore

$$
\operatorname{Aut}(\Delta)^{f}=\mathbb{R}, \quad \operatorname{Aut}(\Delta)^{m}=R .
$$

The subgroup of automorphisms preserving the origin, $\operatorname{Aut}(\Delta, 0)$ is generated by $t \partial_{t}$ over $\mathbb{R}$, therefore it has only a fixed part $\operatorname{Aut}(\Delta, 0)^{f}=\mathbb{R}$.

Collecting all the above results one obtains

$$
\begin{aligned}
\operatorname{Obs}(\Sigma, f)^{f}-\operatorname{Def}(\Sigma, f)^{f}= & \operatorname{Obs}\left(f_{0}\right)^{f}-\operatorname{Def}\left(f_{0}\right)^{f}+\operatorname{Aut}\left(\Sigma_{0}, \nu\right)^{f}-\operatorname{Def}\left(\Sigma_{0}, \nu\right)^{f} \\
= & \operatorname{Obs}\left(\Sigma_{0}, f_{0}\right)^{f}-\operatorname{Def}\left(\Sigma_{0}, f_{0}\right)^{f} . \\
\operatorname{Obs}(\Sigma, f)^{m}-\operatorname{Def}(\Sigma, f)^{m}= & \operatorname{Obs}\left(f_{0}\right)^{m}-\operatorname{Def}\left(f_{0}\right)^{m}+\operatorname{Aut}\left(\Sigma_{0}, \nu\right)^{m}-\operatorname{Def}\left(\Sigma_{0}, \nu\right)^{m} \\
& +\sum_{n=1}^{s-1} R^{-(r+n)}-\sum_{n=1}^{s} R^{n}+T_{p} Y-T_{\nu} \Sigma_{0} \otimes T_{0} \Delta
\end{aligned}
$$

This implies that the virtual fundamental cycle of the fixed locus is the restriction of the natural virtual cycle of the fixed locus $\left[\bar{M}_{g, 1}(Y, d)^{\mathbb{C}^{\times}}\right]^{v i r}$ with the subspace $e v^{-1}(p)^{\mathbb{C}^{\times}}$. The equivariant K-theory class of the virtual normal bundle is given by

$$
\begin{aligned}
N^{v i r}= & N_{\bar{M}_{g, 1}(Y, d)^{\mathrm{C} \times} / \bar{M}_{g, 1}(Y, d)}-T_{p} Y+R \mathbb{L}^{-1} \\
& +\sum_{n=1}^{s-1} R^{-(r+n)}-\sum_{n=1}^{s} R^{n}
\end{aligned}
$$

where $\mathbb{L}$ is the tautological line bundle on $\bar{M}_{g, 1}(Y, d)$ associated to the marked point. Then the residual formula for open Gromov-Witten invariants is

$$
\begin{aligned}
& G W_{g, 1}(d, 1)=(-1)^{s-1} \frac{\prod_{n=1}^{s-1}(r+n)}{s !} \frac{e_{\mathbb{C} \times}\left(T_{p} Y\right)}{\alpha}
\end{aligned}
$$

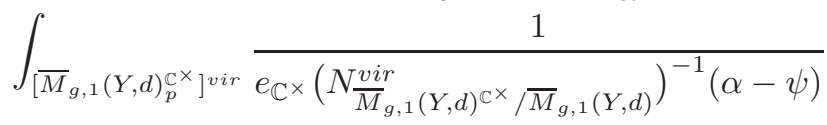


where $\bar{M}_{g, 1}(Y, d)_{p}^{\mathbb{C}^{\times}}$denotes the union of connected components of the fixed locus contained in $e v^{-1}(p)$. Standard formal manipulations show that this formula is equivalent to

$$
G W_{g, 1}(d, 1)=(-1)^{s-1} \frac{\prod_{n=1}^{s-1}(r+n)}{s !} \int_{\left[\bar{M}_{g, 1}(Y, d)\right]_{\mathbb{C} \times}^{v i r}} \frac{e v^{*} \phi_{\mathbb{C} \times}(p)}{\alpha(\alpha-\psi)}
$$

where $\left[\bar{M}_{g, 1}(Y, d)\right]_{\mathbb{C} \times}^{\text {vir }}$ denotes the equivariant virtual cycle of the moduli space, $\phi_{\mathbb{C}^{\times}}(p) \in H_{\mathbb{C}^{\times}}^{*}(Y)$ is the equivariant Thom class of $p \in Y$, and $\alpha=\operatorname{ch}(R)$.

6.4. Comparison with HOMFLY polynomial. The goal of this section is to compare the generating function for the open Gromov-Witten invariants $G W_{g, 1}\left(Y, M_{\epsilon} ; d\right)$ with the HOMFLY polynomial of $(s, r)$-torus knots. It will be shown that large $N$ duality for $(s, r)$ torus knots follows from known results on large $N$ duality for the unknot. The manipulations of enumerative invariants justifying this statement parallel similar manipulations in Chern-Simons theory relating invariants of $(s, r)$ torus knots to colored invariants of the unknot [39, 5].

The main observation is that the Gromov-Witten invariants given in (6.64) for some coprime $(r, s)$ can be expressed in terms of analogous invariants invariants determined by the curve

$$
x=z=w=0
$$

in $X_{0}$ and the associated lagrangian cycles. In order to emphasize the dependence on $(r, s)$, the lagrangian cycles used in the above construction will be denoted by $M_{\epsilon}^{(s, r)}$, and the corresponding invariants by $G W_{g, 1}^{(s, r)}(d, 1)$.

Consider the construction of lagrangian cycles carried out in sections (3.1) (3.3) for a curve $C$ of the form 6.65). By analogy with section 6.1 one can easily check that the lagrangian cycle $M_{\epsilon}^{(1,0)}$ obtained in this case is preserved by any circle action on $Y$ of the form

$$
(x, y, \zeta) \mapsto\left(e^{-i s \varphi} x, e^{-i r \varphi} y, e^{i(r+s) \varphi} \zeta\right)
$$

with $r, s \in \mathbb{Z}$. Moreover, $M_{\epsilon}^{(1,0)}$ intersects the strict transform of $C$ along an orbit of the torus action, obtaining a unique holomorphic circle invariant disk $\mathrm{D}_{0}$ on $Y$ with boundary on $M_{\epsilon}^{(1,0)}$. In this case $\mathrm{D}_{0}$ is smooth and Gromov-Witten invariants with boundary conditions on $M_{\epsilon}^{(1,0)}$ can be constructed in close analogy with [18. Let $\overline{\mathcal{M}}_{g, 1}\left(Y, M_{\epsilon} ; d, k\right)$ be the moduli space of genus $g \geq 0$ stable maps with $h=1$ boundary components mapped to $M_{\epsilon}^{(1,0)}$, in the relative homology class $d\left[C_{0}\right]+k\left[\mathrm{D}_{0}\right] \in H_{2}\left(Y, M_{\epsilon}\right), d \in \mathbb{Z}_{\geq 0}, k \in \mathbb{Z}_{>0}$. In contrast with the previous section, the winding number $k$ will be allowed to take arbitrary values in the present context. Then there is a residual formula of the form

$$
G W_{g, 1}^{(1,0)}(d, k)=(-1)^{k-1} \frac{\prod_{n=1}^{k-1}(r k+n)}{(k-1) !} \int_{\left[\bar{M}_{g, 1}(Y, d)\right]_{\mathbb{C}^{\text {v }}}^{\text {ir }}} \frac{e v^{*} \phi_{\mathbb{C} \times}(p)}{k \alpha(k \alpha-s \psi)}
$$

In particular, setting $k=s$ in equation 6.67), it follows that

$$
G W_{g, 1}^{(s, r)}(d, 1)=s G W_{g, 1}^{(1,0)}(d, s) .
$$

Now define the generating functions with fixed winding numbers 1 , respectively $s$,

$$
F_{1}^{(s, r)}\left(g_{s}, Q, V\right)=\sum_{g \geq 0} \sum_{d \geq 0} g_{s}^{2 g-1} Q^{d} G W_{g, 1}^{(s, r)}(d, 1) \operatorname{Tr}(V)
$$




$$
F_{s}^{(1,0)}\left(g_{s}, Q, V\right)=\sum_{g \geq 0} \sum_{d \geq 0} g_{s}^{2 g-1} Q^{d} G W_{g, 1}^{(1,0)}(d, s) \operatorname{Tr}\left(V^{s}\right)
$$

where the open string Gromov-Witten are defined by residual formulas (6.64), (6.67) with respect to a circle action of the form (6.66).

Large $N$ duality for the unknot yields the following identity [29, Eqn. (5.6)]

$$
F_{q}^{(1,0)}\left(g_{s}, Q, V\right)=\frac{(-1)^{s-1}}{s} \sum_{R} \chi_{R}\left(C_{(s)}\right) e^{i(r / s) \kappa_{R} g_{s} / 2} W_{R}^{(1,0)}(q, Q) \operatorname{Tr}\left(V^{s}\right),
$$

the terms in the right hand side being explained below.

- The sum in the right hand side of (6.69) is over all Young diagrams $R$ and $\chi_{R}\left(\mathcal{C}_{(s)}\right)$ denotes the character of the conjugacy class determined by the vector $\vec{k}=\left(k_{j}\right)_{j \geq 1}$, with $k_{j}=1$ if $j=s$ and $k_{j}=0$ otherwise in the representation determined by $R$. See [29, Sect. 4.1] for more details.

- $W_{R}^{(1,0)}(q, Q)$ is the HOMFLY polynomial colored by the representation $R$ of $U(N)$, expressed as a function of the large $N$ Chern-Simons theory on $S^{3}$,

$$
g_{s}=\left(\frac{2 \pi}{k+N}\right), \quad \lambda=\left(\frac{2 \pi N}{k+N}\right), \quad q=e^{i g_{s}} \quad Q=e^{i \lambda} .
$$

Up to a normalization factor, $W_{R}^{(1,0)}(q, Q)$ is given by the quantum dimension of $R$,

$$
W_{R}^{(1,0)}(q, Q)=Q^{-|R| / 2} \operatorname{dim}_{q}(R) .
$$

where $|R|$ is the total number of boxes in the Young diagram $R$.

- For any Young diagram $R$, the number $\kappa_{R}$ is defined by

$$
\kappa_{R}=|R|+\sum_{i=1}^{l_{R}}\left(l_{i}^{2}-2 i l_{i}\right)
$$

where $l_{R}$ is the number of rows of $R$ and $l_{i}$ is the length of the $i$-th row, $i=1, \ldots, l_{R}$.

As explained in [29, Sect. 3.2] the factor $e^{i m \kappa_{R} g_{s} / 2}$ encodes the framing dependence of colored HOMFLY polynomials, $m$ being the framing of the knot with respect to the canonical framing. The expression $e^{i(r / s) \kappa_{R} g_{s} / 2} W_{R}^{(1,0)}(q, Q)$ in the right hand side of equation (6.69) must therefore be interpreted as a colored HOMFLY polynomial with fractional framing. The relation between quantum knot invariants with fractional framing and residual open string Gromov-Witten invariants has been observed in a similar context in 6 , 8.

Formula (6.69) was initially tested in specific examples for the free term in the $\lambda$-expansion of $W_{R}^{(1,0)}(q, Q)$. The higher order terms were implicitly tested in [8] in the process of finding an enumerative interpretation of the topological vertex [1]. In fact formula (6.69) follows rigorously using more recent results in the mathematical literature 24, 33, 25, on one and two-partition Hodge integrals. Details will be omitted because this is a standard virtual localization computation.

The important fact for the present goal is to note that equations (6.68), (6.69) yield an identity of the form

$$
F_{1}^{(s, r)}\left(g_{s}, q, Q\right)=(-1)^{s-1} \sum_{R} \chi_{R}\left(C_{(s)}\right) e^{i(r / s) \kappa_{R} g_{s} / 2} W_{R}^{(1,0)}(q, Q) .
$$


Now recall that according to [39, Sect 3.3], [5, Eqn (2.43)], the HOMFLY polynomials of $(s, r)$ torus knots is expressed in terms of colored HOMFLY polynomials of the unknot as follows

$$
W_{\square}^{(q, p)}(q, Q)=\sum_{R} \chi_{R}\left(\mathcal{C}_{(s)}\right) e^{2 \pi i(r / s) h_{R}} \operatorname{dim}_{q}(R) .
$$

Next note that

which implies

$$
h_{R}=\frac{N|R|}{2(k+N)}+\frac{\kappa_{R}}{2(k+N)}
$$

$$
e^{2 \pi i(r / s) h_{R}}=e^{i(r / s) \lambda|R| / 2} e^{i(r / s) \kappa_{R} g_{s}} .
$$

Since only diagrams $R$ with $q$ boxes contribute to the right hand side of (6.70), (6.71), it follows that

$$
F_{1}^{(s, r)}\left(g_{s}, q, Q\right)=q^{-r / 2}(-1)^{s-1} W_{\square}^{(s, r)}(q, Q) \operatorname{Tr}(V) .
$$

This is the expected large $N$ duality prediction for torus knots. The factor $(-1)^{s-1}$ reflects a specific choice of orientation of the moduli space of stable maps with lagrangian boundary conditions, as explained above.

\section{REFERENCES}

[1] M. Aganagic, A. Klemm, M. Mariño, and C. Vafa. The topological vertex. Comm. Math. Phys., 254(2):425-478, 2005.

[2] M. Aganagic, M. Marino, and C. Vafa. All loop topological string amplitudes from ChernSimons theory. Commun.Math.Phys., 247:467-512, 2004.

[3] M. Aganagic, H. Ooguri, C. Vafa, and M. Yamazaki. Wall Crossing and M-theory. Publ.Res.Inst.Math.Sci.Kyoto, 47:569, 2011.

[4] M. Aganagic and C. Vafa. Mirror symmetry, D-branes and counting holomorphic discs. 2000. hep-th/0012041.

[5] A. Brini, B. Eynard, and M. Marino. Torus knots and mirror symmetry. arXiv:1105.2012.

[6] S. Cecotti, A. Neitzke, and C. Vafa. R-Twisting and 4d/2d Correspondences. 2010. arXiv:1006.3435.

[7] D.-E. Diaconescu and B. Florea. Large $N$ duality for compact Calabi-Yau threefolds. Adv. Theor. Math. Phys., 9(1):31-128, 2005.

[8] D.-E. Diaconescu and B. Florea. Localization and gluing of topological amplitudes. Commun.Math.Phys., 257:119-149, 2005.

[9] D.-E. Diaconescu, B. Florea, and A. Grassi. Geometric transitions and open string instantons. Adv.Theor.Math.Phys., 6:619-642, 2003.

[10] D.-E. Diaconescu, B. Florea, and A. Grassi. Geometric transitions, del Pezzo surfaces and open string instantons. Adv. Theor.Math.Phys., 6:643-702, 2003.

[11] R. Dijkgraaf, C. Vafa, and E. Verlinde. M-theory and a topological string duality. 2006.

[12] R. Gopakumar and C. Vafa. M-theory and topological strings. I. 1998. hep-th/9809187.

[13] R. Gopakumar and C. Vafa. On the gauge theory/geometry correspondence. Adv. Theor. Math. Phys., 3(5):1415-1443, 1999.

[14] S. Govindarajan, T. Jayaraman, and T. Sarkar. Disc instantons in linear sigma models. Nucl.Phys., B646:498-523, 2002.

[15] S. Hellerman, S. Kachru, A. E. Lawrence, and J. McGreevy. Linear sigma models for open strings. JHEP, 0207:002, 2002.

[16] K. Hori. Linear models of supersymmetric D-branes. pages 111-186, 2000.

[17] A. Iqbal, C. Kozcaz, and C. Vafa. The refined topological vertex. JHEP, 10:069, 2009.

[18] S. Katz and C.-C. M. Liu. Enumerative geometry of stable maps with Lagrangian boundary conditions and multiple covers of the disc. Adv. Theor. Math. Phys., 5(1):1-49, 2001.

[19] S. H. Katz, A. Klemm, and C. Vafa. M theory, topological strings and spinning black holes. Adv.Theor.Math.Phys., 3:1445-1537, 1999.

[20] T. Kawai. String and vortex. Publ.Res.Inst.Math.Sci.Kyoto, 40:1063-1091, 2004. 
[21] S. Koshkin. Conormal bundles to knots and the Gopakumar-Vafa conjecture. Adv. Theor. Math. Phys., 11(4):591-634, 2007.

[22] J. M. F. Labastida, M. Marino, and C. Vafa. Knots, links and branes at large N. JHEP, 11:007, 2000.

[23] J. Li and Y. Song. Open string instantons and relative stable morphisms. In The interaction of finite-type and Gromov-Witten invariants (BIRS 2003), volume 8 of Geom. Topol. Monogr., pages 49-72. Geom. Topol. Publ., Coventry, 2006.

[24] C.-C. M. Liu, K. Liu, and J. Zhou. A proof of a conjecture of Mariño-Vafa on Hodge integrals. J. Differential Geom., 65(2):289-340, 2003.

[25] C.-C. M. Liu, K. Liu, and J. Zhou. A formula of two-partition Hodge integrals. J. Amer. Math. Soc., 20(1):149-184 (electronic), 2007.

[26] K. Liu and P. Peng. New Structure of Knot Invariants. arXiv:1012.2636.

[27] K. Liu and P. Peng. Proof of the Labastida-Marino-Ooguri-Vafa conjecture. arXiv:0704.1526.

[28] K. Liu and P. Peng. On a proof of the Labastida-Marino-Ooguri-Vafa conjecture. Math.Res.Lett., 17:493-506, 2010.

[29] M. Marino and C. Vafa. Framed knots at large N. hep-th/0108064.

[30] P. Mayr. N=1 mirror symmetry and open / closed string duality. Adv. Theor.Math.Phys., 5:213-242, 2002.

[31] D. McDuff and D. Salamon. Introduction to symplectic topology. Oxford Mathematical Monographs. The Clarendon Press Oxford University Press, New York, second edition, 1998.

[32] A. Oblomkov and V. Shende. The Hilbert scheme of a plane curve singularity and the HOMFLY polynomial of its link. arXiv:1003.1568.

[33] A. Okounkov and R. Pandharipande. Hodge integrals and invariants of the unknot. Geom. Topol., 8:675-699, 2004.

[34] H. Ooguri and C. Vafa. Knot invariants and topological strings. Nucl. Phys., B577:419-438, 2000.

[35] R. Pandharipande and R. P. Thomas. Curve counting via stable pairs in the derived category. Invent. Math., 178(2):407-447, 2009.

[36] R. Pandharipande and R. P. Thomas. Stable pairs and BPS invariants. J. Amer. Math. Soc., 23(1):267-297, 2010.

[37] I. Smith and R. Thomas. Symplectic surgeries from singularities. Turkish J. Math., 27(1):231$250,2003$.

[38] I. Smith, R. P. Thomas, and S.-T. Yau. Symplectic conifold transitions. J. Differential Geom., 62(2):209-242, 2002.

[39] S. Stevan. Chern-Simons Invariants of Torus Links. Annales Henri Poincare, 11:1201-1224, 2010.

[40] A. Strominger, S.-T. Yau, and E. Zaslow. Mirror symmetry is T duality. Nucl.Phys., B479:243-259, 1996.

[41] C. H. Taubes. Lagrangians for the Gopakumar-Vafa conjecture. Adv.Theor.Math.Phys., 5:139-163, 2001.

[42] E. Witten. Quantum Field Theory and the Jones Polynomial. Commun.Math.Phys., 121:351, 1989.

[43] E. Witten. Chern-Simons gauge theory as a string theory. In The Floer memorial volume, volume 133 of Progr. Math., pages 637-678. Birkhäuser, Basel, 1995.

${ }^{1}$ NHeTC, Rutgers University, Piscataway, NJ 08854-0849 USA

2 Department of Mathematics, Mit, Cambridge, Ma 02139 USA

3 Jefferson Physical Laboratory, Harvard University, Cambridge Ma 02138 USA 